\title{
Fetal origin of cardiovascular disease : Key role of the kidney
}

Citation for published version (APA):

Sanders, M. W. (2005). Fetal origin of cardiovascular disease : Key role of the kidney. [Doctoral Thesis, Maastricht University]. Universitaire Pers Maastricht. https://doi.org/10.26481/dis.20051021ms

Document status and date:

Published: 01/01/2005

DOI:

10.26481/dis.20051021ms

Document Version:

Publisher's PDF, also known as Version of record

\section{Please check the document version of this publication:}

- A submitted manuscript is the version of the article upon submission and before peer-review. There can be important differences between the submitted version and the official published version of record.

People interested in the research are advised to contact the author for the final version of the publication, or visit the DOI to the publisher's website.

- The final author version and the galley proof are versions of the publication after peer review.

- The final published version features the final layout of the paper including the volume, issue and page numbers.

Link to publication

\footnotetext{
General rights rights.

- You may freely distribute the URL identifying the publication in the public portal. please follow below link for the End User Agreement:

www.umlib.nl/taverne-license

Take down policy

If you believe that this document breaches copyright please contact us at:

repository@maastrichtuniversity.nl

providing details and we will investigate your claim.
}

Copyright and moral rights for the publications made accessible in the public portal are retained by the authors and/or other copyright owners and it is a condition of accessing publications that users recognise and abide by the legal requirements associated with these

- Users may download and print one copy of any publication from the public portal for the purpose of private study or research.

- You may not further distribute the material or use it for any profit-making activity or commercial gain

If the publication is distributed under the terms of Article $25 \mathrm{fa}$ of the Dutch Copyright Act, indicated by the "Taverne" license above, 
FETAL ORIGIN OF CARDIOVASCULAR DISEASE

Key role of the kidney 
FETAL ORIGIN OF CARDIOVASCULAR DISEASE

Key role of the kidney

Thesis, Universiteit Maastricht, Maastricht, The Netherlands ISBN $905278485 \mathrm{X}$

(c) M.W. Buckx-Sanders

Printed by Datawyse/Universitaire Pers Maastricht

Cover designed by E.H. Sanders \& G.E. Fazzi 


\section{FETAL ORIGIN OF CARDIOVASCULAR DISEASE}

Key role of the kidney

\section{Proefschrift}

ter verkrijging van de graad van doctor aan de Universiteit Maastricht, op gezag van de Rector Magnificus,

Prof. mr. G.P.M.F. Mols, volgens het besluit van het College van Decanen, in het openbaar te verdedigen op vrijdag 21 oktober 2005 om 14.00 uur

\section{door}

Maria Wilhelmina Buckx-Sanders geboren op 11 januari 1972 te Susteren

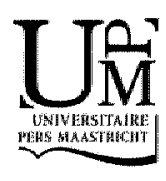




\section{Promotores}

Prof, dr. J.G.R. De Mey

Prof. dr. C.E. Blanco

Beoordelingscommissie

Prof. dr. P.W. De Leeuw (voorzitter)

Dr. G.B. Braam (UMC, Utrecht)

Prof dr. R.A.M.G. Donckerwolcke

Prof. dr. L. Poston (St. Thomas' Hospital, London, UK)

Prof. dr. C.D.A. Stehouwer

Prof. dr. H.A.J. Struijker Boudier

Financial support by the Netherlands Heart Foundation for the publication of this thesis is gratefully acknowledged.

Financial support by Unilever Research and Development, Vlaardingen, The Netherlands for the publication of this thesis is gratefully acknowledged. 


\section{Contents}

General introduction

Chapter 2

Post-natal development of autonomic responses in rat arteries

Chapter 3

Prenatal stress changes rat arterial adrenergic reactivity in a regionally selective manner

Chapter 4

Reduced uteroplacental blood flow alters renal arterial reactivity and glomerular properties in the rat offspring

Chapter 5

High sodium intake increases blood pressure and alters renal function in intrauterine growth retarded rats

General discussion

References

Summary

Samenvatting

Dankwoord

Curriculum vitae

Publications 



\section{General introduction}

\section{General}

Cardiovascular disease is associated with genetic predisposition, smoking, obesity, lack of exercise and diabetes mellitus. In addition, there is now substantial evidence that adverse influences during fetal life increase the susceptibility to cardiovascular, endocrine and metabolic diseases later in life ${ }^{1}$. A suboptimal fetal microenvironment might be involved in what is increasingly recognized as signs of the epigenetics of chronic disease. In the Western world, a compromised fetal environment is often the result of placental insufficiency, which leads to an inadequate delivery of nutrients and oxygen to the developing fetus. The exact mechanisms that link a suboptimal fetal environment to the programming of disease in adult human are largely unknown. Many animal models have been established that support a link between prenatal conditions and the development of disease in the adult. These include maternal caloric and protein restriction ${ }^{2}$, exposure to hypoxia ${ }^{3.4}$ and ligation of the uterine arteries during pregnancy to mimic placental insufficiency ${ }^{5,6}$.

Hypoxia and malnutrition during fetal life were reported to affect the reninangiotensin system, the hypothalamic-pituitary-adrenal axis ${ }^{7}$, vascular endothelial function and sympathetic innervation ${ }^{3,6,8}$ and the development of organ systems like the kidney ${ }^{9,10}$.

Sympathetic nerves innervate the vascular tree, but there are considerable differences in the density and regulatory actions of the sympathetic nervous fibres between different vascular beds. Hyperinnervation of the cardiovascular system precedes the structural and functional changes of the vasculature $^{11,12}$ and eventually leads to an increased peripheral resistance, cardiovascular hypertrophy and high blood pressure.

Thus, sympathetic nerves, neurotransmitter release and the subsequent stimulation of different receptor subtypes are important in maintaining normal cardiovascular dynamics and vascular structure. Adverse intrauterine conditions might alter the development of vascular properties and lead to cardiovascular disease in the adult. In particular renal vascular development and adrenoceptor mediated renovascular responses ${ }^{13}$ might be influenced by disturbed fetal growth. 
Other aspects of renal organogenesis, such as the number of glomeruli can be permanently reduced by adverse influences during fetal life. These structural changes of the kidney may contribute to the development of renal dysfunction ${ }^{14}$ and hypertension ${ }^{15}$ later in life.

\subsection{Development and maturation of the vascular system}

\subsubsection{Mechanisms of vascular development}

The assembly of the vessel wall from its cellular and extracellular matrix components is a critical process in the development and maturation of the cardiovascular system. A functioning circulatory system is necessary for the transport of nutrients and oxygen to, and the removal of wastes from, the developing organ systems of the embryo. Two neovascular processes occur in the embryo. "Vasculogenesis" is the formation of a primitive network, in which angioblasts (undifferentiated endothelial precursor cells) differentiate from the mesoderm and subsequently proliferate and cluster. This process of vasculogenesis (differentiation, proliferation, migration, adherence, etc. of endothelial cells) requires the influence of many regulators. Among these, vascular endothelial growth factor (VEGF), basic fibroblast growth factor (bFGF) and transforming growth factor (TGF- $\beta$ ) play important roles. These regulators also influence "angiogenesis", the process by which a vascular network is extended via the budding of pre-existing vessels ${ }^{16}$.

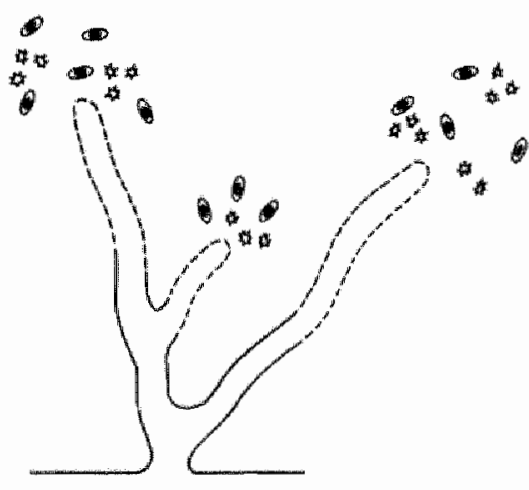

A

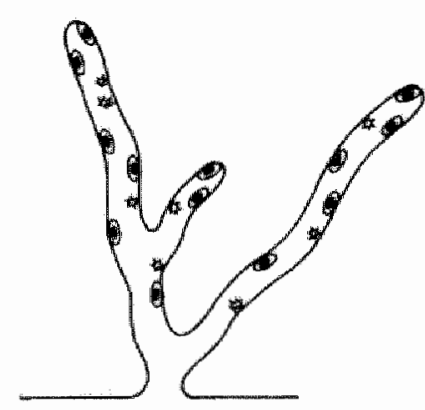

B

Figure 1.1. Schematic representation of angiogenesis (A) and vasculogenesis (B). Angiogenesis is the formation of new vessels by branching (dotted lines) of pre-existing vessels (solid lines) into surrounding tissue. Vasculogenesis is the result of in situ differentiation of endothelial cell precursors (circles, stars) directly into vascular structures ${ }^{\text {t'T }}$ 
Endothelial cells alone can initiate, but not complete, angiogenesis. Periendothelial cells are essential for vascular maturation, but the mechanisms that regulate the subsequent recruitment and differentiation of vascular smooth muscle cells is not well defined. As development proceeds, these cells gain the characteristics of mature smooth muscle cells. This process of "vascular myogenesis" is again controlled by growth factors, such as platelet derived growth factor (PDGF), vascular endothelial growth factor (VEGF), basic growth factor (bFGF), transforming growth factor (TGF- $\beta$ ) and angiopoietin 1. The primary function of the developing vascular smooth muscle cells (VSMC) is to produce and organize cytoskeletal (vimentin. desmin, smoothelin) and extracellular matrix (collagen and elastin) molecules within the developing vessel wall and to serve as a biosynthetic, proliferative and contractile (actin and myosin) component. The developmental program of the VSMCs is intimately linked to vessel wall morphogenesis. The phenotype, which the VSMCs ultimately exhibit shows great plasticity and is indicative of the physical properties of a particular segment of the vasculature ${ }^{18}$. Furthermore, the relationship between the cells of the vessel wall and the matrix they produce is dynamic; it depends on the future function of the vessel and local hemodynamic forces, namely pressure and flow ${ }^{19-22}$. Once, VSMC recruitment and growth during initial vascular myogenesis have occurred, similar signals mediate "arteriogenesis". VSMCs stabilize the vessel wall by migrating alongside preexisting vessels to cover them with a layered muscular coat ${ }^{16.23}$. The structure of concentric and helical layers of VSMCs (tunica media) is given strength and flexibility by the presence of collagen and elastin. The number of cell layers in the tunica media depends on arterial size and type. The thickness of the media increases during intrauterine and postnatal development ${ }^{23}$. This leads to vascular heterogeneity between the same type of vessels of different species, between vessels of different vascular beds in the same species and even between branches of the same vascular bed ${ }^{24}$. The tunica adventitia, the outer layer of the vessel wall contains, besides fibroblasts and collagen and elastin fibres, sensory-motor and sympathetic nerve fibres, which play an important role in controlling vascular tone. 


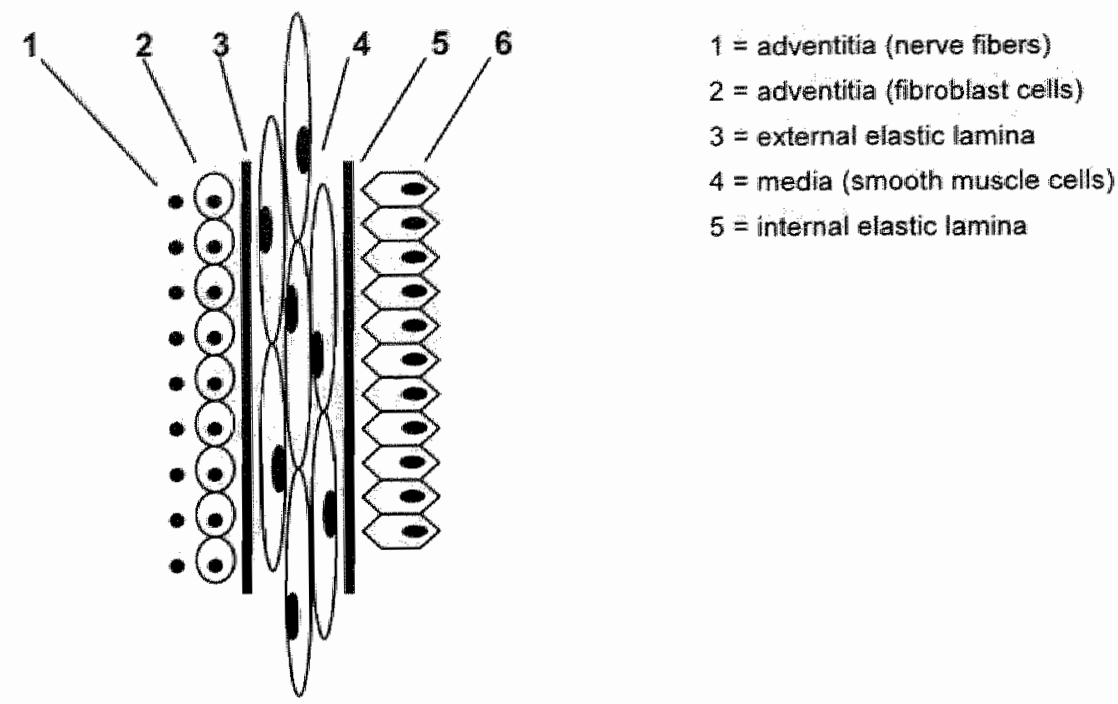

Figure 1.2. Simplified model of the arterial wall components.

\subsubsection{Development of the vascular sensory-motor and sympathetic innervation}

Sensory-motor and sympathetic nerves innervate the vascular tree rather late during development and there are considerable differences in the density and regulatory actions of the nervous fibres between different vascular beds. As mentioned above, during pre- and postnatal maturation blood vessels change dramatically in terms of lumen diameter and wall thickness as functional demand develops. However, little is known about how perivascular nerve fibre development relates to vascular development and about the role of the innervation in the establishment and maintenance of neurotransmitter-receptor populations. Ingrowing sympathetic and sensory-motor nerves might redistribute receptors on the cell surface or can be involved in the up- and down regulation of post-receptor signal transduction system ${ }^{25}$.

First, the precursors of sympathetic neurons arise from undifferentiated neural crest cells that migrate and ultimately differentiate into catecholaminergic neurons. Second, cell number in the autonomic ganglia is refined during a period of cell death when neurotrophic factors determine the number of neuronal precursors and neurons that survive. The final stage of sympathetic development is the establishment and maturation of synaptic connections, which for sympathetic neurons can include alterations in neurotransmitter phenotype. Neurons are initially overproduced and their number is subsequently refined by cell death through competition for trophic factors (nerve growth factor (NGF), NT-3, glial cell line-derived neurotrophic 
factor (GDNF)) synthesized in restricted amounts in target tissues ${ }^{26}$. Nerve growth factor is expressed in target tissues when axons arrive and the presence of nerve growth factors during development differs extensively between different parts of the arterial system ${ }^{27,28}$. This explains the differences in nerve densities between the vascular beds.

In common with sympathetic nerves, sensory-motor nerves are distributed widely in the vasculature. Long term neonatal sympathectomy was followed by an increased number of CGRP-containing nerves ${ }^{29}$ and experiments after long term neonatal denervation of sensory-motor nerves with capsaicin have shown that these nerves have a trophic influence on the development and function of sympathetic nerves ${ }^{30}$. The absence of sympathetic nerves might have increased the availability of nerve growth factor (NGF) for CGRP. containing nerves and vice versa. These findings indicate that the presence of sensory-motor nerves is a prerequisite for an efficient development and function of sympathetic nerves. This in turn is necessary for maintaining optimal function of sensory-motor nerves and the development of adrenergic vascular function.

\subsubsection{Regulation of vascular tone}

The endothelium, the cells lining the inner wall of the vasculature, the tunica media as well as the tunica adventitia are very important in maintaining vascular tone. Vasoactive substances released by the endothelium and by periarterial nerves can either contract or relax the vascular smooth muscle cells in the tunica media. Mechanical stimuli or substances that bind to their receptors can elicit contraction by increasing intracellular calcium concentrations. Receptor activation leads to the activation of phospholipase C (PLC) that cleaves phosphatidyl biphosphate $\left(P P_{2}\right)$ into inositol triphosphate $\left(\mathrm{I}_{3}\right)$ and diaglycerol $(\mathrm{DAG})^{31}$. Furthermore, receptor activation may open receptor-operated calcium channels (ROC) in the sarcolemma directly or indirectly through second messengers. $\mathrm{IP}_{3}$ induces the release of calcium from the sarcoplasmic reticulum (SR) through second messenger operated calcium channels $(S M O C)^{32,33}$. DAG activates protein kinase $C$ (PKC) which increases the calcium sensitivity of the contractile apparatus and calcium influx. This mediates the interaction of actin and myosin filaments ${ }^{34.35}$. 


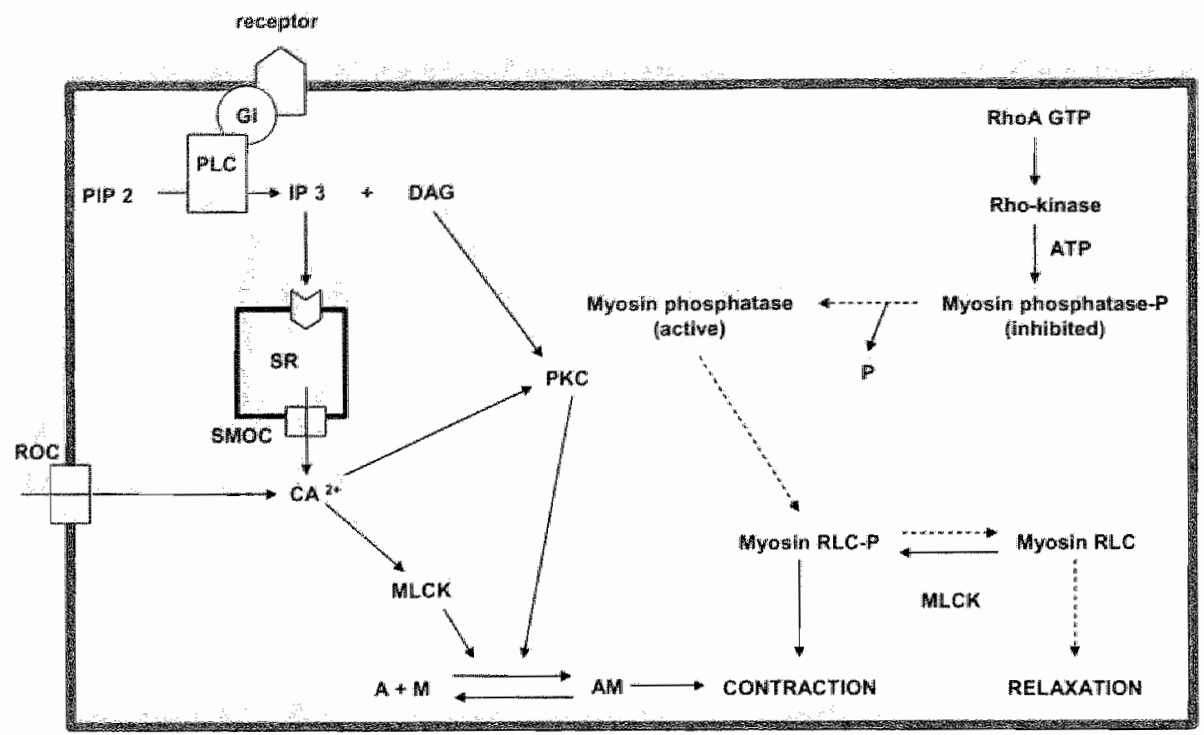

Figure 1.3 . Schematic picture of the $[\mathrm{Ca} 2+]$-dependent and -independent regulatory mechanisms for smooth muscle contraction.

However, contractility of the smooth muscle is not only regulated by fluctuations in cytoplasmic calcium, but also by membrane potentialindependent pharmacomechanical coupling. Smooth muscle myosin is regulated by phosphorylation/dephosphorylation of the myosin regulatory light chains (RLC) by calcium-calmodulin-regulated myosin light chain kinase (MLCK) and myosin phosphatase (MLCP). The major calcium-independent pathway that increases myosin activity is through the activation of Rhokinase by RhoA GTP, phosphorylation of the regulatory subunit of myosin phosphatase (MLCP) by Rho-kinase, resulting in inhibition of MLCP activity and increased myosin RLC phosphorylation. These mechanisms operate in parallel with and independently of the activation of MLCK by calcium released from the sarcoplasmatic reticulum by $\mathbb{I P}_{3}$ or by calcium influx ${ }^{36}$. Binding of a substance to its receptor can also lead to relaxation of the smooth muscle cells. Receptor binding can stimulate adenylyl cyclase (AC) through activation of stimulatory $\left(G_{s}\right)$ GTP binding regulatory proteins. This results in an increased production of cyclic adenosine monophosphate (CAMP), which reduces calcium sensitivity of the contractile machinery, stimulates intracellular calcium uptake and calcium extrusion mechanisms and causes hyperpolarization. This eventually leads to relaxing responses ${ }^{37-}$ 39. Furthermore, activation of guanylyl cyclase (GC) generates cyclic guanosine monophosphate (cGMP). Like CAMP, cGMP exerts vasodilating responses in the vascular smooth muscle ${ }^{40}$. 


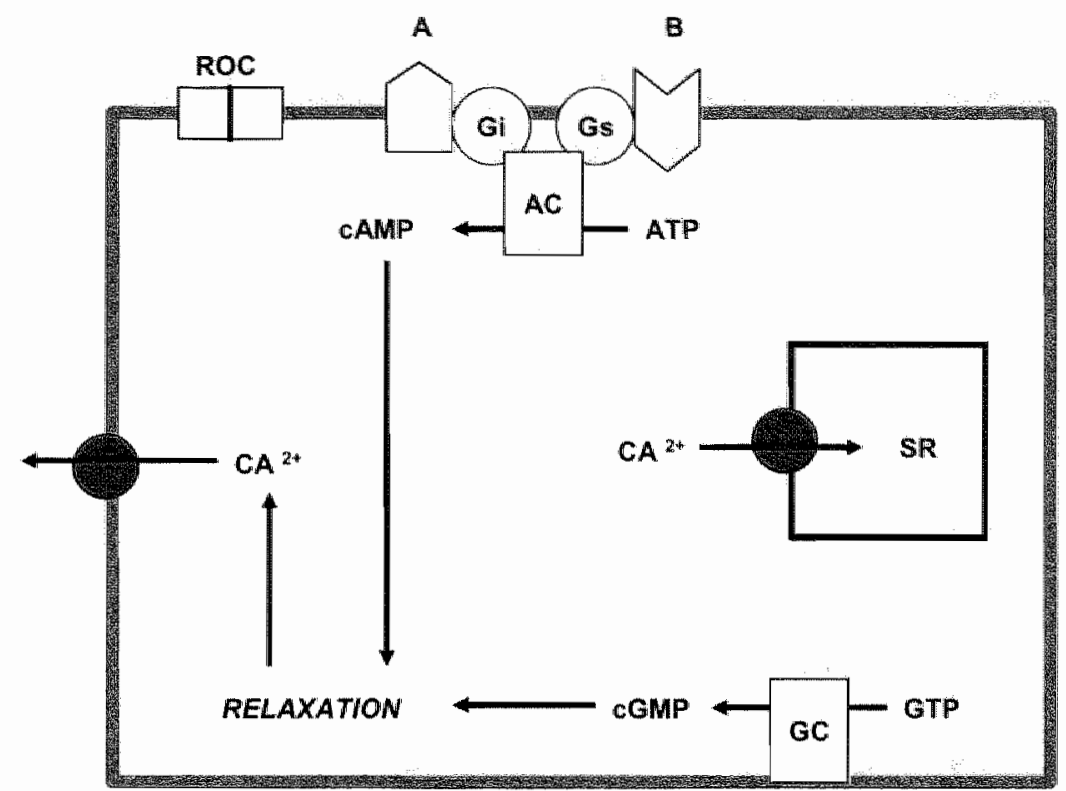

Figure 1.4. Schematic picture of the cyclic nucleotide system.

The endothelium acts as a regulator of vascular tone by releasing various vasoactive substances: vasodilators (nitric oxide, prostacyclin, endotheliumderived hyperpolarizing factor, bradykinin, adrenomedullin, C-natriuretic peptide), vasoconstrictors (endothelin-1, angiotensin-II, thromboxane $A_{2}$, prostaglandins, hydrogen oxide and free radicals). One of the most important substances that the endothelium produces is nitric oxide (NO). NO is released from the endothelium either by chemical activation (e.g. acetylcholine) or by mechanical activation as a result of the wall shear stress exerted by the flowing blood. NO is synthesized within the endothelial cells from L-arginine by endothelial nitric oxide synthase ${ }^{41}$.

To which extent nerves surrounding the vasculature can contract or relax vascular smooth muscle cells depends largely on the density of their fibres and on the width of the synaptic clefts. Sensory-motor nerves mainly release calcitonin gene-related peptide (CGRP). In the vasculature CGRP activates adenylyl cyclase, increases CAMP content and causes relaxation ${ }^{42}$. Norepinephrine is the main neurotransmitter released by sympathetic nerves. Norepinephrine can bind to $\alpha_{1}$ and $\beta$-adrenoceptors (and to a lesser extent to $\alpha_{2}$-adrenoceptors). Binding of norepinephrine to $\beta$-adrenoceptors causes vasorelaxation through the activation of AC. Activation of $\alpha_{1-}$ adrenoceptors contracts the vascular smooth muscle via calcium influx through L-VOC and by activation of PLC and RhoA/Rho kinase ${ }^{36,43}$. 


\subsubsection{Vascular disease}

Endothelial dysfunction appears to be a common and early feature of (cardio)vascular disease. In addition to its function as a vasodilator, NO released from endothelial cells is also a potential inhibitor of the aggregation and adhesion of platelets to the vascular wall. NO also controls the expression of proteins involved in atheroma formation by decreasing the expression of the chemo-attractant protein MPC-1 and of surface adhesion molecules such as CD11/CD18, P-selectin, VCAM-1 and ICAM-1. NO also reduces vascular permeability and decreases the rate of oxidation of lowdensity lipoprotein (LDL). This prevents the pro-atherogenic effect of LDL and inhibits the proliferation of vascular smooth muscle cells. Thus a reduction in the bio-availability of NO leading to endothelial dysfunction can contribute to the pathogenesis of cardiovascular disease.

Essential hypertension, a common cardiovascular disorder, is initiated and sustained by overactivity of the sympthetic nervous system. Among others, possible mechanisms include increased sympathetic nerve firing rates, altered neuronal norepinephrine reuptake, diminished arterial baroreflex buffering of sympathetic nerve traffic and facilitation of norepinephrine release by neurohumoral factors such as angiotensin $1{ }^{44,45}$. Sympathetic factors are also involved in the progression of cardiovascular structural alterations. The sympathetic innervation can cause hyperplasia of the vascular wall by influencing the proliferation of smooth muscle cells and decreasing the synthesis of collagen. Additionally, by promoting vascular smooth muscle cell replication, activation of the sympathetic nervous system can favour the atherogenic process, because smooth muscle cell replication precedes their migration to the intima and transformation to macrophages. This is a key element in the cascade of events leading to the established atherosclerotic plaque. Furthermore, sympathetic hyperreactivity may increase cardiac and renal load by increasing blood viscosity, because under conditions of increased sympathetic drive ${ }_{n}$ hematocrit is increase $d^{45}$.

\subsection{The development and function of the kidney}

\subsubsection{Anatomical development of the kidney}

During embryonic and fetal development in mammals there are three different pairs of renal organs: the pronephros, the mesonephros and the metanephros. The metanephros forms the permanent kidney in the adult. The pronephric tubule extends into the intermediate mesoderm of the mesonephros to form the Wolffian duct. The mesonephros functions as a primitive filtering organ in the embryo with the formation of nephron tubules and glomeruli, which connect to the Wolffian duct, but the tubules lack a loop of Henle. The ductall system of the mesonephros ultimately contributes to the 
tubule system of the male and female reproductive tracts. The metanephros develops from an outbranch of the Wolffian duct called the ureteric bud which extends into the mass of undifferentiated metanephric mesenchyme. Interactions between the ureteric bud epithelium and the metanephric mesenchyme result in the formation of the collecting duct system and nephrons of the permanent kidney ${ }^{46}$.
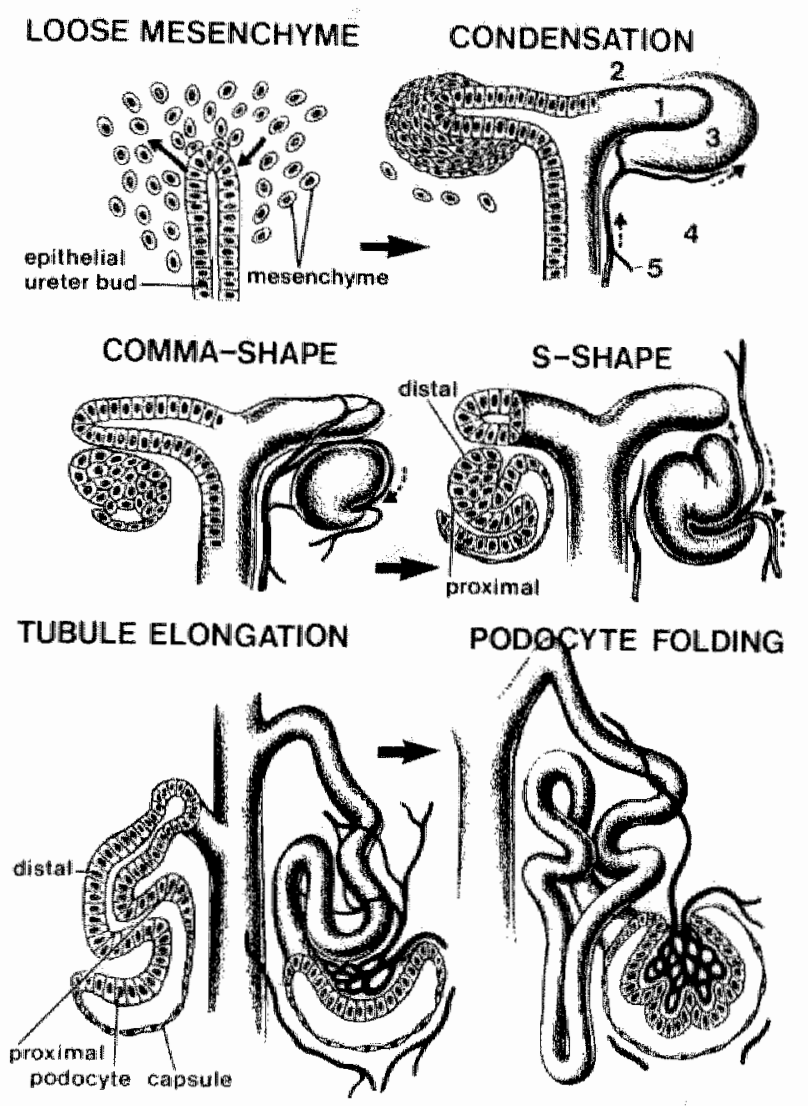

Figure 1.5. Schematic representation of nephrogenesis ${ }^{17}$. 
The process of nephrogenesis (=metanephrogenesis) is characterized by active cell proliferation and hypertrophy, accompanied by large scale apoptosis to eliminate unwanted cells. This is regulated by the coordinated action of a wide variety of growth factors and receptors (insulin-like, epidermal, transforming, nerve, hepatocyte, fibroblast, platelet-derived and vascular-endothelial growth factors, bone morphogenic proteins, leucocyte inhibitory factor), proto-oncogenes, transcription factors, suppressor genes, extracellular matrix components and degrading enzymes and extracellular matrix ligands and receptors. Nephrogenesis is complete before birth in humans and sheep, but not in many other species, like the rat where it proceeds until two weeks after birth ${ }^{47}$.

\subsubsection{Origin of the kidney vasculature}

The kidneys are highly vascularized organs that in the adult animal receive about $25 \%$ of the cardiac output. Blood vessel formation begins early in kidney morphogenesis. Kidney vascularization and mephrogenesis occur simultaneously and are synchronized with one another. The origin of the kidney vasculature has not been fully defined. Angiogenesis and vasculogenesis have both been postulated.

It is believed that vascularization of the kidney is achieved mainly by angiogenesis. The vascular system of the kidney derives from endothelial cells that invade the organ early during nephrogenesis. It has also been proposed that the vascularization of the kidney is initiated by a pair of vessels originating from the embryonic aorta. These vessels invade the metanephric mesenchyme presumably following the branching ureter. As the ureter induces nephron differentiation, endothelial cells originating from the invading vessels penetrate the lower cleft of the S-shaped bodies and generate the glomerular capillaries. Since endothelial cells possess the information necessary to form a capillary network, given the appropriate extracellular signals, it is likely that a precise guiding system is provided by angiogenic factors and extracellular matrix components (growth factors, proteases, integrins, adhesive glycoproteins, cell surface receptors and mechanical forces) ${ }^{17}$.

\subsubsection{Functional development of the kidney}

In the fetus, the regulation of fluid and electrolyte homeostasis is accomplished primarily by the placenta and the haemostatic role of the kidney is not well defined. However, the fetal metanephric kidney produces a large volume of dilute urine, which is a major input into the amnionic fluid. The amnionic fluid is essential as an aqueous environment for the symmetrical growth of the fetus and correct lung development. Factors that prevent urine being produced by the fetal kidneys might result in lack of amnionic fluid and subsequent fetal growth abnormalities. The kidney, in 
addition to its excretory function, is an important endocrine organ in utero. Major hormones produced by the kidney include erythropoietin (EPO), which stimulates the bone marrow to make red blood cells and the metanephros also expresses components of the renin-angiotensin system. Renin is an enzyme that is synthesized predominantly in the juxtaglomerular cells of the afferent arterioles in the kidney. When released, renin cleaves angiotensin I from the substrate angiotensinogen, a protein mainly produced in the liver. Angiotensin I is subsequently hydrolyzed by angiotensin converting enzyme (ACE) to form the active angiotensin II. Angiotensin II acts a mitogen in the developing kidney. In addition to stimulating cell growth directly, angiotensin II may also regulate the synthesis of growth factors important for renal development ${ }^{48}$.

\subsubsection{Physiological anatomy of the adult kidney}

\section{Renal circulation}

The renal arteries enter the kidney through the helium and then branch progressively to ultimately form the afferent arterioles, which lead to the glomerular capillaries, where large amounts of fluid and solutes are filtered to begin urine formation. The renal circulation is unique in that it has two capillary beds, the glomerullar and the peritubular capillaries, which are arranged in series and separated by the efferent arterioles that help to regulate the hydrostatic pressure in both sets of capillaries. High hydrostatic pressure in the glomerular capillaries causes rapid fluid filtration, whereas a much llower hydrostatic pressure in the peritubular capillaries allows rapid fluid reabsorption. By adjusting the resistance of the afferent and efferent arterioles, the kidneys can regulate the hydrostatic pressure in both the glomerular and the peritubular capillaries. This permits them to change the rate of glomerular filtration and/or the tubular reabsorption in response to homeostatic demands. The peritubular capillaries empty into the vessels of the venous system and ultimately form the renal vein, which leaves the kidney beside the renal artery and ureter. 


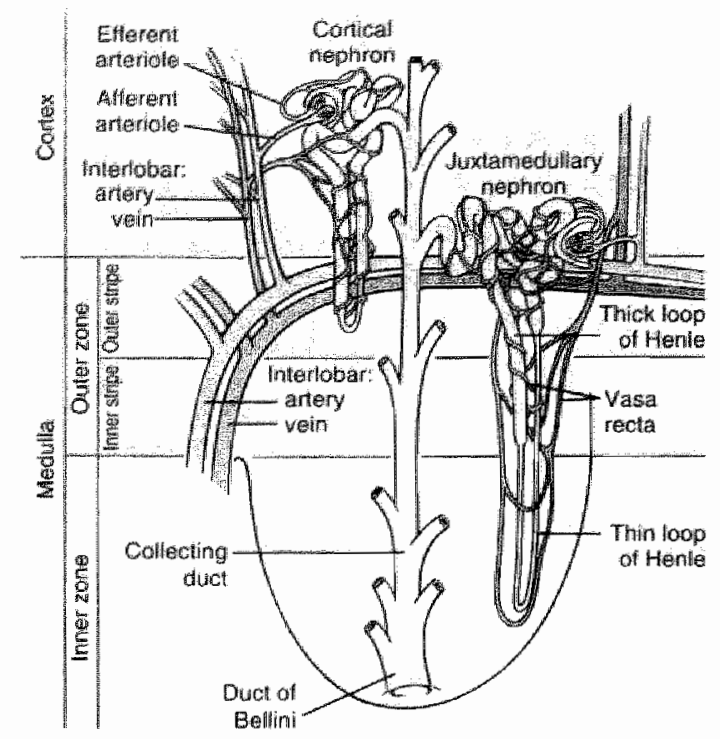

Figure 1.6. Relation between blood vessels and tubular structures and differences between corticall and juxtamedullary nephrons ${ }^{4 \varphi}$.

\section{The nephron}

The nephron is the urine-producing unit of the kidney. The adult kidney cannot regenerate new nephrons. Therefore, with renal injury, disease or normal ageing, there is a gradual decrease in the number of nephrons.

Each nephron contains (1) a cluster of glomerular capillaries called the glomerulus, through which large amounts of fluid are filtered from the blood and (2) a long tubule in which the filtered fluid is converted into urine. The glomerulus contains a network of anastomising glomerular capillaries that have high hydrostatic pressures. The total glomerulus is enclosed in Bowman's capsule. Fluids filtered from the glomerular capillaries flows into Bowman's capsule and then into the proximal tubule, which lies in the cortex of the kidney. From the proximal tubule, fluid flows into the loop of Henle, which descends into the renal medulla. Each loop consists of a descending and an ascending limb. At the end of the ascending limb is a short segment, known as the macula densa. Beyond the macula densa, fluid enters the distal tubule, which lies in the cortex. The distal tubule is followed by the connecting tubule and the cortical collecting tubule, which leads to the cortical collecting duct. The collecting ducts merge to form progressively larger ducts that eventually empty into the renal pelvis. However, nephrons are not all uniform and two distinct types are found, called cortical nephrons 
and juxtamedullary nephrons, depending on the location of their glomeruli in the cortex. Cortical nephrons have short loops of Henle that penetrate only a short distance into the medulla. Juxtamedullary nephrons have glomeruli that lie deep in the renal cortex near the medulla. These nephrons have long loops of Henle that descend deeply into the medulla ${ }^{49}$.

\subsubsection{General function of the kidneys in the adult}

The kidneys perform their most important functions by filtering the plasma and removing substances from the filtrate at variable rate, depending on the needs of the body. After this, the kidneys clear unwanted substances from the filtrate by excreting them in the urine, while returning substances that are needed back to the body. However, it is important to recognize that the kidneys serve multiple functions; Excretion of metabolic waste products, foreign chemicals, drugs and hormone metabolites, regulation of water and electrolyte balances, of arterial pressure, of acid-base balance, of erythrocyte production, and of 1,25-dihydroxy vitamin $D_{3}$ production and glucose synthesis ${ }^{49} \cdot 1 \cdot 2.6$. Glomerular filtration rate and renal blood flow

\section{Glomerular filtration}

Urine formation begins with the filtration of large amounts of fluid through the glomerular capillaries into Bowman capsula. Glomerular capillaries are relatively impermeable to proteins, so that the glomerular filtrate is essentially free of proteins and cellular elements. These capillaries have a much higher rate of filtration than other capillaries, because of a high glomerular hydrostatic pressure and a large filtration coefficient. The glomerular filtration rate (GFR) is determined by (1) the sum of the hydrostatic and colloid osmotic forces across the glomerular membrane (net filtration pressure) and (2) the glomerular filtration coefficient $\left(K_{f}\right)$.

GFR $=K_{f} \times$ net filtration pressure

Under normal conditions, the concentration of proteins in the glomerular filtrate is so low that the colloid osmotic pressure in the Bowman's capsule is considered to be zero. Some diseases lower $K_{f}$ by reducing the number of functional glomerular capillaries (thereby reducing the surface area for filtration) or by increasing the thickness of the glomerular capillary membrane and reducing its hydraulic conductivity. For instance, chronic hypertension and diabetes mellitus gradually reduce $K_{f}$ by increasing the thickness of the glomerular capillary basement membrane ${ }^{49}$. 


\section{Renal blood flow}

Blood flow supplies the kidneys with nutrients and removes waste products. The kidneys receive an extremely high blood flow compared with other organs and it greatly exceeds its needs. The purpose of this additional flow is to supply enough plasma for the high rates of glomerular filtration that is necessary for precise regulation of body fluid volume and solute concentrations. The mechanisms that regulate renal blood flow (RBF) are linked to the control of GFR and the excretory function of the kidneys.

RBF is determined by the pressure gradient across the renal vasculature (the difference between renal artery (RA) and renal (RV) vein hydrostatic pressure), divided by the total renal vascular resistance (TRVR).

$\mathrm{RBF}=(\mathrm{RAP}-\mathrm{RVP}) / \mathrm{TRVR}$

The total renal vascular resistance is controlled by the sympathetic nervous system, various hormones and local internal renal control mechanisms. Although changes in arterial pressure have some influence on renal blood flow, the kidneys have effective mechanisms for maintaining RBF and GFR relatively constant, a process called autoregulation ${ }^{49}$.

\section{Control of glomeruar filtration rate and renal blood flow}

All the blood vessels in the kidneys are richly innervated by sympathetic nerve fibres. Strong activation of the renal sympathetic nerves can constrict the renal arterioles and decrease RBF and GFR. Moderate or mild sympathetic stimulation has little influence on RBF and GFR and in a healthy situation, there appears to be little sympathetic tone to the kidneys.

Angiotensin $I I$ is a powerful vasoconstrictor that preferentially constricts efferent arterioles. This raises glomerular hydrostatic pressure and reduces RBF. Angiotensin II formation usually occurs in circumstances associated with decreased arterial pressure or volume depletion, which decreases GFR. By constricting efferent arterioles, angiotensin II prevents a decrease in glomerular hydrostatic pressure and GFR, to maintain a normal excretion of waste products, such as urea and creatinine. Constriction of the efferent arterioles by angiotensin II also reduces RBF. This contributes to decreased flow through the peritubular capillaries, which increases reabsorption of sodium and water. In turn, this helps to restore blood volume and blood pressure. Other vasoactive substances, like $N O$, prostaglandins $\left(P G E_{2}\right.$ and $\mathrm{PGI}_{2}$ ) and bradykinin also appear to be important for preventing excessive vasoconstriction of the kidneys and allowing them to excrete normal amounts of sodium and water.

To perform the function of autoregulation, the kidneys have a feedback mechanism that link changes in sodium chloride concentration at the macula 
densa with the control of renal arteriolar resistance. The macula densa cells sense changes in volume delivery to the distal tubule: A decreased GFR may slow the flow rate in the loop of Henle, causing an increase in reabsorption of sodium and chloride ions in the ascending loop of Henle and thereby reducing the concentration of sodium chloride at the macula densa. This initiates a signal from the macula densa that has two effects: (1) it decreases resistance of the afferent arterioles, which raises glomerular hydrostatic pressure and returns GFR toward normal and (2) it increases renin release from the juxtaglomerular cells of the afferent and efferent arterioles. Renin release from these cells increases the formation of angiotensin I and subsequently angioten $\sin 1 \mathrm{I}^{49}$.

\section{Filtration fraction}

The fraction of the renal plasma flow that is filtered is referred to as the filtration fraction (FF = GFR / RBF). The filtration fraction can be either increased by raising GFR or by reducing $\mathrm{RBF}^{49}$.

\section{Methods to quantify glomerular filtration rate and renal blood flow}

The rate at which different substances are cleared from the plasma provide a useful way of quantisation the effectiveness with which the kidneys excrete various substances. If a substance is freely filtered (like water) and is not bound to plasma proteins nor secreted or metabolically altered in the renal tubules, then the rate at which that substance is excreted in the urine is equal to the filtration rate of the substance by the kidneys. A substance that fits these criteria is inulin, a polysaccharide molecule ${ }^{50}$. Inulin is not produced in the body and must be administered intravenously to measure GFR. Inulin is not the only substance that can be used for determining GFR. Other substances that have been used to estimate GFR include creatinine and radioactive iothalamate.

Theoretically, if a substance is completely cleared from the plasma, the clearance rate of that substance is equal to the total RBF. There is no known substance that is completely cleared by the kidneys. One substance, however, PAH (para-aminohippurate), is about 90\% cleared from the plasma. Therefore, the clearance of $\mathrm{PAH}$ can be used as an approximation of $\mathrm{RBF}^{49}$.

\subsubsection{Role of the kidneys in the regulation of blood pressure}

In response to extracellular fluid expansion, the blood volume and arterial pressure rise. The rising blood pressure causes the kidneys to excrete the excess of extracellular fluid and salt, the main determinant of extracellular fluid volume, returning the pressure back towards normal. An increased extracellular fluid volume increases blood volume, which rises mean 
circulatory filling pressure. This in turn increases venous return of blood to the heart and causes atrial stretch. In response to atrial stretch the heart secretes atrial natriuretic factor (ANF), which acts on the kidneys to increase sodium excretion and GFR, to antagonize renal vasoconstriction and to inhibit renin secretion ${ }^{51}$.

An excess of salt in the body increases the asmolarity of body fluids and this in turn stimulates the thirst centre, making a person drink more water to dilute the extracellullar salt concentration back to normall. The increased osmolarity in the extracellullar fluid also stimulates the hypothalamic-posterior pituitary gland to secrete antidiuretic hormone (vasopressin). This hormone causes the kidneys to reabsorb increased quantities of water from the renal tubular fluid, thereby diminishing the excreted volume of urine while raising the extracellular fluid volume. An addition to this mechanism of pressure control is the role of the renin-angiotensin system. A fall in arterial pressure causes prorenin molecules in the juxtaglomerular cells to split and release renin. Renin cleaves angiotensin I from the substrate angiotensinogen, a protein mainly produced in the liver. Angotensin I is subsequently hydrolyzed by angiotensin converting enzyme (ACE) to form the active angiotensin 11 . Angiotensin II is an extremely powerful vasoconstrictor. Vasoconstriction occurs intensely in the arterioles and much less in the veins. Constriction of the arterioles increases total peripheral resistance, which increases arterial pressure. Also, the mild constriction of the veins promotes increased venous return of blood to the heart, thereby helping the heart pump against the increasing pressure. However, the renin-angiotensin vasoconstrictor system requires about 20 minutes to become fully active. Thus it acts somewhat slower on pressure control than the nervous reflexes and the sympathetic nervous system. The second, long-term and more powerful mechanism, by which angiotensin II can increases arterial pressure is to act directly on the kidneys to decrease the excretion of salt and water. The constriction of renal arterioles diminishes the flow though the kidneys. As a result, less fluid filters though the glomeruli into the tubules. Also, the slow flow of blood in the peritubular capillaries reduces their pressure, which allows rapid osmotic reabsorption of fluid from the tubules. All this leads to a decreased excretion of urine. In addition, angiotensin It has an effect on the tubular cells themselves to increase tubular reabsorption of sodium and water.

Angiotensin $I I$ is also a powerful controller of aldosteron secretion. The subsequent function of aldosteron is to cause an increase in sodium reabsorption in the tubules, which is followed by an increased sodium concentration in the extracellular fluid, an increased extracellular fluid volume and an elevation of the arterial pressure. Then, the increased arterial pressure causes an increased blood flow through the kidneys, which reduces the secretion rate of renin. This results in a reduced retention of salt and water and returns the extracellular fluid volume and the arterial pressure 
back to normal. Thus, the renin-angiotensin system is an automatic feedback mechanism that helps maintain the arterial pressure at a normal level ${ }^{49}$.

\subsubsection{The kidney and cardiovascular disease}

Chronic kidney disease is frequently associated with cardiovascular disease. Most of the traditional risk factors of cardiovascular disease, such as older age, diabetes mellitus, hypertension, left ventricular hypertrophy and low high-density lipoprotein (HDL) cholesterol are highly prevalent in chronic kidney disease ${ }^{52}$. It has been proposed that a low number of nephrons and enlarged glomeruli increase the risk of both hypertension and progressive renal disease $\mathrm{e}^{53}$. After removal of large portions of the kidney or after reduced nephron endowment at birth ${ }^{54}$, the remaining nephrons undergo adaptive changes that lead to an increased blood flow, increased glomerular filtration and an increased urine output in the surviving nephrons. The exact mechanisms responsible for these changes are not well understood, but involve hypertrophy as well as functional changes that decrease vascular resistance and tubular reabsorption. These changes permit a person to excrete normal amounts of water and solutes. However, the functional changes may eventually lead to further injury of the glomeruli and cause glomerulosclerosis (replacement of normal tissue with connective tissue) These sclerotic lesions can eventually destroy the glomeruli, which leads to a further loss of kidney function and further adaptive changes. This slowly progressing vicious circle can eventually lead to end-stage renal disease and a person must be placed on dialysis treatment ${ }^{49}$.

Epidemiological and experimental data have also shown that high levels of albumin excretion in the urine (albuminuria) are associated with an increased incidence of cardiovascular disease. Although the renal mechanisms of albuminuria are not well elucidated, albuminuria may be a marker of renal vascular abnormalities and predispose a person to develop hypertension and cardiovascular disease ${ }^{52,55}$.

\subsection{Fetal programming of cardiovascular disease}

\subsubsection{The hypothesis of fetal programming}

Many epidemiological studies have been performed to show an association between fetal growth retardation and cardiovascular disease in the adult. This association is the basic concept of the hypothesis that is put forward by Barker as the "fetal origins of adult disease". The growth and development of the mammalian fetus is completely dependent on the nutrients and oxygen supplied by the mother. An inadequate maternal metabolism, inappropriate maternal food intake or disturbed maternal/fetal flow can impair the nutrient and oxygen supply to the fetus. The fetus can respond to these potentially 
harmful stimuli (stress) by altering the maturation of the cardiovascular system and the metabolic function of organs for survival purposes. However, these early changes which might be beneficial for the fetus in the short term may prove damaging in the long-term and predispose an individual to the development of disease in adulthood.

\subsubsection{Birth weight and the fetal programming of adult disease}

Most epidemiological studies on fetal programming provide data that associate reduced weight at birth with cardiovascular disease in adulthood.

The large number of studies that have investigated the link between size at birth and cardiovascular diseases like, coronary heart disease, stroke, hypertension and Non-linsulin Dependent Diabetes Mellitus have been extensively reviewed ${ }^{56-59}$. In addition, many animal studies have been conducted that confirm an association between low birth weight and the occurrence of cardiovascular disease in adulthood ${ }^{4,60-62}$.

However, birth weight is only a crude measure of fetal growth and intrauterine nutrition and oxygen supply. Furthermore, catch-up growth after birth may also contribute to the development of disease in the adult. Studies in humans, which describe the effects of prenatal exposure to the Dutch famine on health in later life, found indications that the effects of undernutrition depend upon its timing during gestation and on the tissues and systems undergoing critical periods of development at that time. Additionally, they suggest that undernutrition during gestation may permanently affect adult health without affecting the size of the baby at birth. This implies that adaptations that enable the fetus to continue to grow may nevertheless have adverse consequences for health in later life ${ }^{63}$.

\subsubsection{Catch-up growth and the fetal programming of adult disease}

In addition to retarded fetal growth, there is now evidence that its effects on later cardiovascular disease are modified by postnatal growth. Accelerated postnatal growth might be an attempt to restore overall body dimensions and organ weights. However, studies in humans found that the highest blood pressures were found in those who had low birth weight but were tall as adults $^{64}$. Another study showed that the highest rates of coronary heart disease were found in those who were thin at birth but whose weight caught up through accelerated weight gain in early childhood ${ }^{65}$. The same investigators also found that hypertension may originate through retarded growth in utero followed by accelerated postnatal growth ${ }^{66}$. A possible link between catch-up growth and cardiovascular disease is that retarded fetal growth leads to permanently altered structure and/or function of tissues, which can not be restored after birth. Subsequent accelerated growth may lead to excessive demand on these tissues and eventually to the development of cardiovascular disease. Thus, catch-up growth can have 
either beneficial or detrimental effects, in terms of longevity. 'Buying' survival to reproduction at the expense of adaptations that are detrimental in old age is a strategy which is likely to have positive rather than negative evolutionary value $^{67}$.

\subsection{Mechanisms behind the fetal programming of adult disease}

Controlled animal-based studies are beginning to provide insight into the molecular, cellular and systemic mechanisms that contribute to the different manifestations of fetal programming and will be discussed below.

\subsubsection{Hypoxia during fetal development}

Chronic hypoxia during pregnancy is a common insult to fetal development and is associated with intrauterine growth restriction and increased perinatal mortality and the subsequent development of cardiovascular disease. The largest group of persons that are at risk to be subjected to chronic hypoxia in utero are residents at high altitude. There has been a long history of studies of pregnancy, fetal and neonatal life at high altitude. It was found that the effects of high altitude on birth weight is as great or greater than that associated with low maternal weight gain, smoking, primiparity or preeclampsia. The decline in birth weight is principally due to a slowing of fetal growth in the third trimester. The incidence of pre-eclampsia is increased at high altitude and this likely contributes to the altitude-associated birth weight decline. Studies show that chronic hypoxia alters maternal systemic and uteroplacental vascular adjustments to pregnancy. Cardiac output is lower at high altitude, probably as the result of lower blood volume and/or higher systemic vascular resistance. The higher systemic vascular resistance in pregnant humans might contribute to higher blood pressures by a greater myogenic tone, altered production of local regulators of vascular tone (imbalance of vasoconstrictor and vasodilators) and/or a lack of compensatory organ remodelling that occurs in normal pregnancy ${ }^{68}$. Several animal models have been developed to gain more insight into the mechanisms of hypoxia-associated intrauterine growth restriction and its consequences later in life. A study in rats demonstrated that prenatal chronic hypoxia $\left(10,5 \% \mathrm{O}_{2}\right.$ during day $15-21$ of gestation) reduced birth weights and increased the susceptibility of the adult heart to injury due to ischemia and reperfusion. However, the effects of hypoxia during fetal life on the adult hearts were not evident until they were stressed ${ }^{69}$. Investigations in the chicken embryo showed that chronic moderate hypoxia during fetal life results in disproportionate growth retardation and in an increased sympathetic innervation of peripheral arteries. In addition, chronic exposure to hypoxia induced changes in endothelial function (especially the nitric- 
oxide -dependent component $)^{3.70}$. Sympathetic hyperinnervation and vascular smooth muscle hyperplasia may be involved in the development of hypertension in experimental animals ${ }^{11,71}$ and possibly humans ${ }^{44}$. It has also been found that low birth weight in humans is associated with increased sympathetic activity ${ }^{72}$. Already as neonates, subjects with low birth weight exhibit endothelial dysfunction that persists into childhood and adult life ${ }^{73}$. Thus, both hypoxia induced sympathetic hyperinnervation and endothelial dysfunction may contribute to the development of cardiovascular disease later in life.

\subsubsection{Malnutrition during fetal development}

Undernutrition is still an important health problem in underdeveloped countries and epidemiological evidence suggests that cardiovascular disease can also be programmed by exposure to a poor diet during intrauterine life $^{57}$. As mentioned above, studies in humans that were born during the Dutch famine showed that undernutrition during gestation affects health in later life ${ }^{63}$. However, the mechanisms underlying the programming of cardiovascular disease by maternal undernutrition are likely to be multifactorial and complex. Although other models of maternal undernutrition have been used, the most widely reported and characterized animal model of nutritional programming has utilized the feeding of low protein diets in rat pregnancy. A substantial protein restriction in utero led to a reduction in nephron number ${ }^{74}$, glomerular enlargement and hypertension ${ }^{75}$ and increased urinary output and urinary albumin excretion in the offspring ${ }^{76}$. In addition, it has been observed that rats submitted to intrauterine protein restriction have an increased capacity to retain salt and water and this might be a mechanism involved in the development of hypertension ${ }^{77}$.

When pregnant rats were given the synthetic glucocorticoid, dexamethasone, the offspring had low birth weight, a reduced number of glomeruli and developed adult albuminuria and hypertension ${ }^{78}$. Protein restriction reduces the activity level of placental $11 \beta$-hydroxysteroid dehydrogenase $(11 \beta \mathrm{HSB})$, an enzyme that normally inactivates maternal cortisol (in humans) or corticosterone (in rats) and a reduced activity of this enzyme increases the exposure of the fetus to maternal glucocorticoids ${ }^{79}$. Thus, an excess of glucocorticoid exposure to the fetus can impair renal development and function. However, the mechanism by which glucocorticoids exert this effect is not known. In mature animals glucocorticoids stimulate the entry of calcium into vascular smooth muscle cells, promoting vasoconstriction and increase peripheral resistance ${ }^{80}$. Indirectly, glucocorticoids may act on blood pressure by regulating the reninangiotensin system ${ }^{81}$.

It is also well established that the renin-angiotensin system plays an important role in the development of the kidney ${ }^{48}$. For example, it was 
observed that maternal protein restriction suppresses intrarenal renin gene and protein expression in the newborn offspring and plasma renin activity $^{82,83}$. Woods et al. also found a decrease in angiotensin $\|$ in the kidneys at the time of birth ${ }^{82}$. Angiotensin $I I$ is important for normal nephrogenesis ${ }^{84}$ and interfering with the production of angiotensin $\|$ or blocking its effects on receptors might alter normal renal development ${ }^{65}$.

Another mechanism that links uterine undernutrition to the development of cardiovascular disease later in life is an impaired endothelium dependent vasodilatation. Although there are discrepancies related to differences in the feeding protocol, several studies have demonstrated impaired responses to different endothelium-dependent agonists in different vascular beds in intrauterine undernourished rats ${ }^{85}$. Whereas some reports showed preserved responses to sodium nitroprusside in the vascular beds from intrauterine undernourished rats ${ }^{36}$, others demonstrated that the response to a NO-donor substance was reduced in rats submitted to low protein diet in utero ${ }^{87,88}$. It has been suggested that intrauterine undernutrition promotes alterations in nitric oxide pathways ${ }^{88}$. In addition, a reduced activity of nitric oxide synthase (NOS) associated with increased concentrations of reactive oxygen species, such as superoxide anion, which leads to the scavenging of nitric oxide, might play an important role in endothelium dysfunction in intrauterine undernourished rats ${ }^{86,89}$.

\subsubsection{Consequences of placental insufficiency}

Pregnancy requires a major cardiovascular and circulatory adaptation of the maternal organism to provide adequate perfusion of the uterus, which supplies oxygen and nutrients to the growing conceptus. If the uterine perfusion is restricted, a deficiency in the delivery of both nutrients and oxygen to the uterus, placenta and fetus occurs. This can cause fetal growth retardation and might lead to the development of cardiovascular disease in the adult offspring. Intrauterine growth restriction within the Western world is more likely the result of impaired uteroplacental perfusion rather than of maternal undernutrition or hypoxemia. In addition, several problems are associated with investigating the mechanisms of the fetal programming of adult disease in human populations, such as inadequate recording of confounding variables, loss of follow-up studies and genetic predisposition. Several maternal risk factors are known to be associated with increased risk of fetal growth retardation, including maternal smoking, primiparity, maternal hypertensive disorders and previous fetal growth retardation.

Diverse animal model have been developed to investigate the problem of uteroplacental insufficiency. For example in sheep, chronic placental insufficiency can be induced by daily umbilico-placental embolization using microspheres ${ }^{62,90}$ or by instrumentation with a vascular occluder ${ }^{91}$. It has been shown that umbilico-placental embolization down-regulates $11 \beta$ - 
hydroxysteroid dehydrogenase type 2 gene expression and enzyme activity in the fetal sheep kidney, which may contribute to the development of hypertension ${ }^{92}$. This is supported by human observations that up to $10 \%$ of essential hypertensive patients have a prolonged plasma half-life of cortisol and appear to have relative $11 \beta$-hydroxysteroid dehydrogenase type 2 deficiency ${ }^{93}$. Another feature of fetal growth restriction in both sheep and humans after placental insufficiency is a lower plasma glucose level. This might be due to a reduced placental perfusion, an inadequate placental glucose transfer, a decreased gluconeogenesis or a change in insulin sensitivity ${ }^{94}$.

The experimental model of ligation of one or both uterine arteries is also frequently used to investigate the consequences of placental insufficiency in the $\mathrm{rat}^{61}$. It was found that placental insufficiency induced by reduced perfusion in the pregnant rat results in low birth weight offspring predisposed to development of hypertension ${ }^{95}$. Placental insufficiency due to bilateral uterine artery ligation also leads to type 2 diabetes as a result of an impaired glucose sensing or defective oxidative phosphorylation of glucose, the primary signal eliciting insulin secretion by the $\beta$-cell ${ }^{96}$. Thus, it is apparent that placental insufficiency has major impacts on the fetus with consequences for the cardiovascular and metabolic development up to adulthood.

\subsection{Aim and outline of the thesis}

The rat model of uteroplacental insufficiency has many similarities to human fetal growth retardation, like decreased levels of glucose, insulin, aminoacids and oxygen; it can be used to circumvent the difficulties of human studies and to elucidate the underlying mechanisms of fetal programming of adult disease. The present thesis aims to determine whether intrauterine stress (IUS), due to a reduced uteroplacental blood flow, results in longlasting alterations of renovascular function and glomerular structure, which may blunt structural and functional compensatory mechanisms in the kidney and lead to the development of hypertension later in life.

The aim of the study in chapter 2 was to compare developmental changes in the pharmacological control of different parts of the peripheral arterial system and to asses the contribution of adrenergic and sensory motor nerves to these changes and their potential regional heterogeneity. We therefore studied regionally different rat arteries at $6,10,15,21$ days and 12 weeks of age. We determined their size and nerve densities, their contractile and relaxing responses to exogenously supplied agonists and stimuli that trigger the release of endogenous neurotransmitters. 
In the study, described in chapter 3 , we induced an experimental intrauterine stress (IUS) by bilateral ligation of the uterine arteries at day 13 of pregnancy (early in gestation) in rats and investigated the consequences of IUS for adrenergic vascular responses in the offspring. To evaluate whether IUS affects regionally different vascular beds in a different manner, mesenteric resistance, renal, femoral and saphenous arteries were taken from the 21 day old offspring and studied with pharmacological methods. Changes in pattern and density of cathecholamine-containing nerves were also assessed using fluorescence immunohistochemistry and $\mathrm{a}_{1-}$ adrenoceptors were approached by radioligand binding.

In chapter 4 we determined the long-term consequences of IUS for the adult offspring. IUS was induced by bilateral ligation of the uterine arteries at day 17 of pregnancy (late in gestation) and renal vascular adrenergic responses of the 21 days old offspring were evaluated. At the age of 12 weeks, the male adult offspring was instrumented for measuring glomerular filtration rate, renal blood flow and blood pressure. Some of the experiments were performed after unilateral nephrectomy at 21 days of age to investigate whether IUS blunts structural and functional compensatory mechanisms in the kidney.

Adult rats, which survived IUS, were put on a high salt diet (in drinking water) to study their renal compensatory capacities and to test if they were more susceptible to develop hypertension. The results of this study are explained in chapter 5 .

In the general discussion (chapter 6) the results of our experimental studies are discussed and general conclusions and perspectives are formulated. 


\section{Abstract}

We studied the maturation of autonomic responses of four types of arteries of rats during post-natal life, to understand normal development of arterial autonomic structure and function within different vascular beds.

Organ chamber, morphometry and (immuno)histochemistry studies were used to determine changes in the structure and function of arteries and their sensory-motor- and adrenergic innervation in regionally different vascular beds. We isolated mesenteric resistance arteries (MrA), renal arteries (RA), femoral arteries (FA) and saphenous arteries (SA) from male Wistar rats at $6,10,15,21$ days and 12 weeks of age.

Diameter, wall thickness and maximal contractile responses increased markedly with age in all four preparations. Catecholamine- and calcitonin gene-related peptide (CGRP)-containing nerves and regional differences in their density were already present at 6 days of age.

Before contractile responses to $\alpha_{1}$-adrenergic receptor stimulation (phenylephrine) appeared, catecholamine-containing nerves and responses to sympathetic nerve stimulation (electrical field stimulation) had already developed and did not markedly change after this period of development.

In the RA, FA and $\mathrm{SA}_{\text {s }}$ maximal active wall stress remained constant during development and relaxing responses to exogenous and endogenous CGRP decreased with age, while the density of CGRP-containing nerve fibers did not change during postnatal maturation. Relaxing $B$-adrenergic responses (isoproterenol) decreased during development in RA, FA and SA. The decreased relaxing responses to neurogenic and adrenergic activators of adenylyl cyclase (AC) were accompanied by a reduced sensitivity to direct stimulation of AC with forskolin.

In contrast to the other arteries, MrAs did not demonstrate a loss of AC mediated responses and maximal active wall stress and autonomic nerve density kept increasing with age.

In summary, the development of autonomic arterial responses occurs in a distinct way in regionally different vascular beds. The mechanism probably involves rearrangement of receptor-expressions and changes in the subsequent signal transduction systems. 


\section{Post-natal development of autonomic responses in rat arteries}

Marijke W. Sanders, Gregorio E. Fazzi, Carlos E. Blanco, Jo G. R. De Mey

\section{Introduction}

Hemodynamic, neural, circulating and locally produced factors can influence growth and maturation of vascular beds as they adapt their structure and function to the requirements of the organ that is supplied.

Although there is specialization in the structure and function of regionally different blood vessels, most vascular beds are under control of the autonomic nervous system (afferent nerve fibers containing a variety of peptides and efferent sympathetic nerve fibers containing cathecholamines) ${ }^{97}$ "but nerve densities vary markedly between vascular territories $^{98}$.

Little is known about the effect of normal development on the role of autonomic innervation in the establishment and maintenance of receptor populations. Ingrowing sympathetic and sensory-motor nerves might redistribute receptors on the cell surface or can be involved in the up- and down regulation of post-receptor signal transduction system.

Even though, the fetus can be exposed to catecholamines during prenatal development ${ }^{99-101}$ "in the rat, the maturation of the sympathetic nervous system does not become established until well after birth ${ }^{102}$ and afferent and efferent nerves are heterogeneously distributed along the vascular tree.

To evaluate postnatal development of arterial autonomic function changes in peptidergic, adrenergic and sympathetic responses were recorded in regionally different arteries taken from rats of varying postnatal ages $(6,10$, 15,21 days and 12 weeks after birth).

We focused on mesenteric resistance, renal, femoral and saphenous arteries and evaluated vascular responses with pharmacological methods. Changes in pattern and density of cathecholamine-containing nerves and sensory-motor nerves were also assessed using fluorescence- and immunohisachemistry. 


\section{Materiais and methods}

\section{Animals}

Experiments were approved by the local ethical committee for animal research. Animals had free access to pelleted food and tap water and were maintained on a 12 -h light/dark cycle at $21^{\circ} \mathrm{C}$.

Wistar rats (Charles River) weighing 200-250 g were mated and conception was confirmed by the vaginal smear method. Day 1 of pregnancy was defined as the day immediately following the night during which males were present. Rats delivered spontaneously at $22 \pm 1$ days and litter size $(12 \pm 1)$ and birth weight $(5.39 \pm 0.15 \mathrm{~g})$ of the offspring were determined within an hour after birth. At day $6,10,15$ and 21 days and 12 weeks of age, animals were killed by decapitation or cervical dislocation.

\section{Arterial reactivity}

Regionally different arterial segments were isolated from $6,10,15$ and 21 day old and from adult (12 weeks) male Wistar rats. Two stainless-steel wires (diameter $40 \mu \mathrm{m}$ ) were inserted in the lumen of the arterial segments, which were then mounted in an organ chamber between an isometric force transducer and a displacement device (Danish Myotechnology by J.P. Trading, Denmark). The organ chamber was filled with Krebs-Ringer bicarbonate solution which was maintained at $37^{\circ} \mathrm{C}$ and continuously aerated with $95 \% \mathrm{O}_{2}$ and $5 \% \mathrm{CO}_{2}$. Before the actual experiments started, arterial segments were stretched to their individual optimal lumen diameter for mechanical performance, i.e. the diameter at which maximal contractile responses to potassium $(40 \mathrm{mM})$ were obtained ${ }^{403}$. In most experiments a mesenteric resistance, renal, femoral and saphenous arterial segment from one animal were mounted in an organ chamber and studied in parallel. Mesenteric resistance arteries from 6-day-old rats were too small to mount in an organ chamber. The pharmacological agents that were used included calcitonin gene-related peptide (CGRP), capsaicin, phenylephrine, isoproterenol and forskolin. In adult rat mesenteric resistance arteries, relaxing responses to isoproterenol , CGRP and capsaicin are not altered by mechanical removal of the endothelium ${ }^{104-106}$. At the start of the experiments, all arterial preparations were exposed to $1 \mu \mathrm{M}$ capsaicin during 20 minutes to stimulate and permanently desensitize sensory-motor nerves, while it does not affect efferent fibers of the autonomic nervous system ${ }^{107.108}$. In adult rat mesenteric arteries, relaxing responses to capsaicin are prevented by CGRP 8-37, indicating that they are mediated by endogenously released CGRP $^{105-107}$. Sympathetic neuroeffector mechanisms were studied using electrical field stimulation (EFS; $0.25-32 \mathrm{~Hz}, 2 \mathrm{msec}, 85 \mathrm{~mA}$ ) via platinum electrodes, which were placed in the axial direction, and a stimulator designed by the local technical department. Contractile responses were 
expressed as active wall tension $(\mathrm{N} / \mathrm{m})$ or as percentage of the maximal tissue contraction, induced by a mixture of $125 \mathrm{mM}$ potassium and $10 \mu \mathrm{M}$ phenylephrine.

Relaxing responses were investigated during contraction induced by $40 \mathrm{mM}$ $K^{*}$ to blunt effects of the tested compounds on sarcolemmal $K^{*}$-channels ${ }^{109}$, in order to concentrate on ultimate stimulatory influences on adenylyl cyclase. Relaxing responses were expressed as percentage change of the pre-existing contractile tension. Effects of isoproterenol on $\alpha_{1}$-adrenoceptors were prevented by $1 \mu \mathrm{M}$ prazosin.

\section{Morphometric analysis}

Following reactivity measurements, arterial segments were fixed at optimal diameter by replacing the organ bath solution with preheated phosphatebuffered formaldehyde $\left(4 \%, \mathrm{pH} 7.4,37^{\circ} \mathrm{C}, 30\right.$ minutes). The tissues were transferred to $70 \%$ ethanol and imbedded in paraffin. Thin cross-sections (4 $\mu \mathrm{m}$ ) were stained with Lawson solution (Boom B.V., Meppel, the Netherlands). Media cross-sectional area (MCSA) was measured by subtracting the area enclosed by the internal elastic lamina from the area enclosed by the media-adventitial border using video images generated by a Zeiss Axioscope (Zeiss, Germany), a standard CCD camera (Sony) and commercially available software (JAVA 1.4, Jandel Scientific, Corte Madera, CA, USA). Lumen radius (r) was calculated from the internal circumference assuming a circular cross section and media thickness (Mt) was derived using the following formula: $M t=-r+\sqrt{ }\left(r^{2}+\operatorname{CSA} / \pi\right)$.

\section{Staining of perivascular nerves}

Sensory-motor nerves of arterial segments were stained with a rabbit anti-rat CGRP antibody. Vessels were fixed in phosphate buffered ( $\mathrm{pH} 7.4$ ) formaldehyde $(4 \%)$ overnight at room temperature and washed in ethanol $(70 \%)$. Endogenous peroxidase activity was blocked by treatment with $\mathrm{H}_{2} \mathrm{O}_{2}$ $(0.3 \%)$ for 2 hours at room temperature, following which preparations were washed with ethanol $(70 \%)$. After subsequent treatment with Triton $X-100$ $(0.2 \%$ in PBS) vessels were incubated with the primary CGRP antibody $(1: 3000)$ overnight at room temperature. On the next day, preparations were washed again with Triton $X-100$ for 1 hour and incubated with the secondary antibody for 2 hours (swine anti-rabbit peroxidase, 1:200) and washed again with Triton $X-100$. Finally, preparations were incubated with $1.2 \mathrm{mmol} / \mathrm{L}$ diaminobenzidine and $0.03 \% \mathrm{H}_{2} \mathrm{O}_{2}$ for 15 minutes at room temperature, dehydrated to xylene ( 30 mins.) and embedded in Entellan.

To demonstrate the presence of sympathetic nerves, whole mount preparations of arterial segments were incubated in $2 \%$ glyoxilic acid and $10 \%$ sucrose in phosphate buffer for 10 minutes at room temperature. After this, segments were air dried (90 seconds), stretched at $100^{\circ} \mathrm{C}$ for 4 
minutes, and enclosed with entellan and a cover slip. Glyoxylic acid-induced fluorescence was visualized with fluorescent microscopy (Nikon Diaphot, BA 470-DM 455 filter, Nikon EF2 camera) ${ }^{110}$.

\section{Drugs and solutions}

Krebs-Ringer bicarbonate buffer contained (in mmol/L): $\mathrm{NaCl} 118.5, \mathrm{MgSO}_{4}$ - $7 \mathrm{H}_{2} \mathrm{O} 1.2, \mathrm{KH}_{2} \mathrm{PO}_{4} 1.2, \mathrm{NaHCO}_{3} 25.0, \mathrm{CaCl}_{2} 2.5$ and glucose 5.5. A 40 mmol/L $\mathrm{K}^{*}$ solution was prepared by replacing part of the $\mathrm{NaCl}$ by an equimolar amount of $\mathrm{KCl}$. Phosphate-buffered solution consisted of 0.1 $\mathrm{mol} / \mathrm{L} \mathrm{NaH} \mathrm{PO}_{4} \cdot \mathrm{H}_{2} \mathrm{O}$ and $0.1 \mathrm{~mol} / \mathrm{L} \mathrm{Na}_{2} \mathrm{HPO}_{4} \cdot 2 \mathrm{H}_{2} \mathrm{O}$. Calcitonin generelated peptide (CGRP) was obtained from BACHEM (Bubendorf, Switzerland), capsaicin and phenylephrine from Sigma chemical co (St. Louis, MO, USA), isoproterenol and forskolin from ICN Pharmaceuticals (Costa Mesa, CA, USA) and Lawson solution from Boom b.v. (Meppel, The Netherlands). CGRP, phenylephrine and isoproterenol were dissolved in distilled water and capsaicin and forskolin in ethanol (100\%).

\section{Data analysis}

Maximal responses (Emax) to phenylephrine and electric nerve stimulation for each artery were expressed as active wall tension (isometric force divided by twice the segment length; $N / m$ ), as wall stress (tension divided by media thickness) and as percentage of maximal tissue contraction (\%). Relaxing responses were expressed as percentage relaxation of contraction induced by $40 \mathrm{mM}$ potassium.

Concentration response curves were analyzed in terms of sensitivity ( $\mathrm{pD} 2=$ - $\log E C 50$ ) by fitting individual concentration-response data to a non-linear sigmoid regression curve and interpolation (Graphpad Prism version 2.01, Graphpad Software Inc.). Differences between findings in arteries from rats of different ages were tested with Student $t$ test. A value of $p<0.05$ was considered statistically significant. Data are presented as mean \pm S.E.M. 


\section{Results}

Table 2.1 summarizes optimal diameters, media cross-sectional area and media to lumen ratio of four types of arteries of $6,10,15$ and 21 days and 12 weeks old male Wistar rats. At all ages renal and femoral arteries have a larger optimal diameter as well as a larger media cross-sectional area than the smaller mesenteric resistance and saphenous artery. For all arteries lumen diameter, media cross-sectional area and wall to lumen ratio increased markedly with age.

Table 2.1. Structural properties of isolated rat mesenteric resistance (MrA), renal (RA), femoral (FA) and saphenous (SA) arteries.

\begin{tabular}{|c|c|c|c|c|}
\hline & MrA & RA & FA & SA \\
\hline Day 6 & & $(n=8)$ & $(n=8)$ & $(n=7)$ \\
\hline OD & N.A. & $187 \pm 46$ & $250 \pm 7$ & $165 \pm 9$ \\
\hline MCSA & N.A. & $5.55 \pm 0.56$ & $6.86 \pm 0.31$ & $2.87 \pm 0.32$ \\
\hline M/L & N.A. & $5.83 \pm 0.23$ & $6.12 \pm 0.15$ & $5.97 \pm 0.38$ \\
\hline Day 10 & $(n=3)$ & $(n=4)$ & $(n=4)$ & $(n=4)$ \\
\hline OD & $107 \pm 4$ & $273 \pm 28$ & $264 \pm 15$ & $165 \pm 8$ \\
\hline MCSA & $2.23 \pm 0.75$ & $11.11 \pm 1.03$ & $12.61 \pm 1.91$ & $5.03 \pm 0.63$ \\
\hline$M / L$ & $7.02 \pm 0.06$ & $7.76 \pm 0.44$ & $7.79 \pm 0.32$ & $7.67 \pm 0.56$ \\
\hline Day 15 & $(n=7)$ & $(n=7)$ & $(n=7)$ & $(n=7)$ \\
\hline OD & $154 \pm 6$ & $307 \pm 12$ & $357 \pm 8$ & $234 \pm 18$ \\
\hline MCSA & $4.12 \pm 0.82$ & $17.13 \pm 1.14$ & $21.00+11.68$ & $9.06 \pm 1.71$ \\
\hline$M / L$ & $8.57 \pm 1.45$ & $9.18 \pm 0.60$ & $8.93 \pm 0.57$ & $8.92 \pm 0.68$ \\
\hline Day 21 & $(n=8)$ & $(n=22)$ & $(n=10)$ & $(n=9)$ \\
\hline OD & $182 \pm 1$ & $362 \pm 21$ & $600 \pm 37$ & $307 \pm 13$ \\
\hline MCSA & $5.11 \pm 0.42$ & $22.54 \pm 1.46$ & $33.09+2.06$ & $16.22 \pm 1.08$ \\
\hline$M / L$ & $8.19 \pm 0.38$ & $9.71 \pm 0.23$ & $10.66 \pm 0.64$ & $10.71 \pm 0.79$ \\
\hline Adult & $(n=6)$ & $(n=7)$ & $(n=5)$ & $(n=5)$ \\
\hline$O D$ & $284 \pm 13$ & $647 \pm 35$ & $607 \pm 28$ & $467 \pm 88$ \\
\hline MCSA & $15.88 \pm 1.78$ & $93.43 \pm 5.52$ & $93.18 \pm 5.31$ & $50.87 \pm 6.83$ \\
\hline$M / L$ & $10.30+0.67$ & $10.67 \pm 0.57$ & $13.25 \pm 0.59$ & $15.78+1.50$ \\
\hline
\end{tabular}

OD $(\mu \mathrm{m})$, optimal diameter. MCSA ( $\times 1000 \mu \mathrm{m} 2)$, cross-sectional area of the media. M/L, media to lumen ratio (media thickness/radius $x 100$ ). Values are mean $\pm S . E . M_{n}, \mathrm{n}$ denotes number of animals studied. 


\section{Arterial reactivity}

Maximal contractile responses (Tmax) to a mixture of $125 \mathrm{mM}$ potassium and $10 \mu \mathrm{M}$ phenylephrine increased with age in all four preparations (fig. 2.1.a). At all ages, Tmax was comparable for the four types of arteries, despite differences in diameter and media mass. Maximal wall stress (WSmax) increased with age in the mesenteric artery, while it did not change after 15 day of age in the other tested vessels (fig. 2.1.b). WSmax was comparable for the renal and femoral arteries. After 15 days of age WSmax in the saphenous artery was larger than in the renal and femoral arteries and smaller than in the mesenteric resistance artery.

A

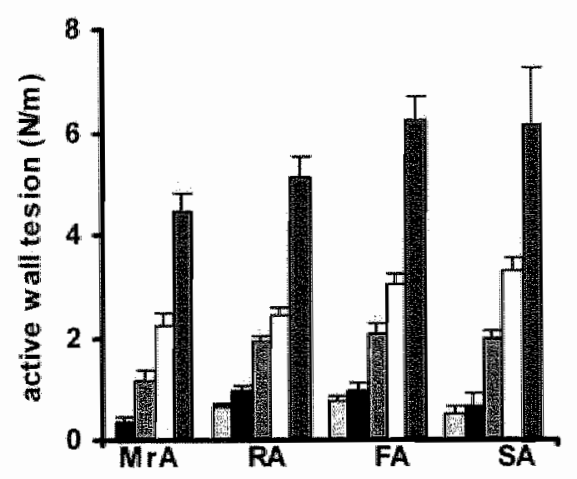

B

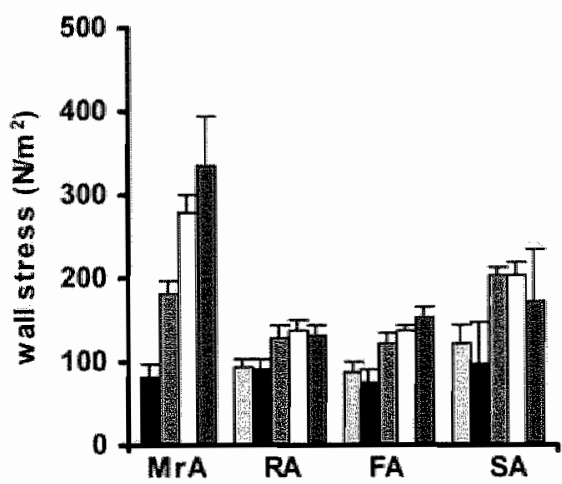

Figure 2.1. Maximal active wall tension (Tmax) (A) and maximal active wall stress (Wsmax) (B). induced with a mixture of $125 \mathrm{mM} \mathrm{K}+$ and $10 \mu \mathrm{M}$ phenylephrine, in first order mesenteric resistance (MrA), renal (RA), femoral (FA) and saphenous arteries (SA). day $15, \square$ day 21 , adult. Data are mean $\pm S . E . M .(n=3$ to 22$)$.

Arterial contractile responses to sympathetic nerve stimulation (electrical nerve stimulation after exposure to capsaicin) gradually increased during development in all tested segments and active wall tension reached highest values at the age of 12 weeks (fig. 2.2.a). When responses to sympathetic nerve stimulation were expressed as percentage of Tmax, it was noted that contractile responses did not increase with age (fig. 2.2.b).

Contractile responses of the renal and femoral artery to nerve stimulation expressed as percentage of Tmax remained smaller during development than in the mesenteric resistance and saphenous artery (fig. 2.2.b). In the saphenous artery, which is a side branch of the femoral artery, sympathetic nervous contractile responses were considerably larger than in the femoral artery. 
A

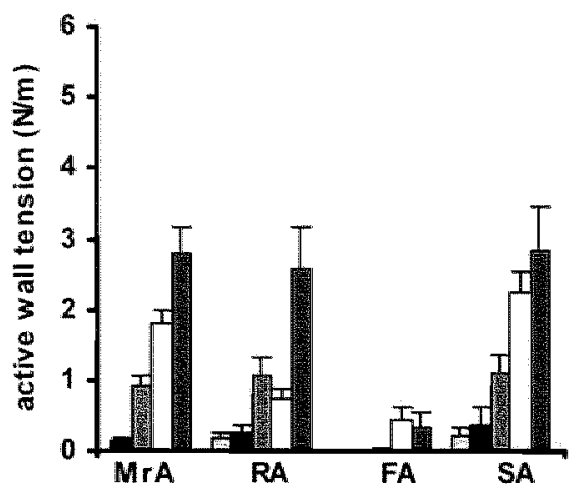

B

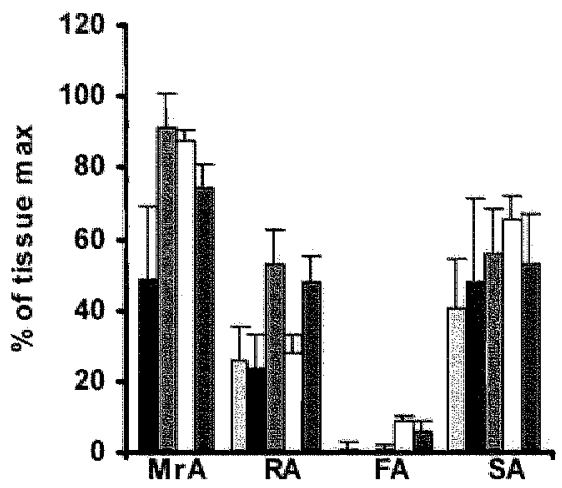

Figure 2.2. Maximal contractile responses to electrical field stimulation (EFS) in absolute values (A) and expressed as \% of Tmax (B), after 20 min. exposure to $1 \mu \mathrm{M}$ capsaicin, in first order mesenteric resistance (MrA), renal (RA), femoral (FA) and saphenous arteries ( $S A$ ). - day 10, of day $15, \square$ day 21 , adult. Data are mean \pm S.E.M.

A

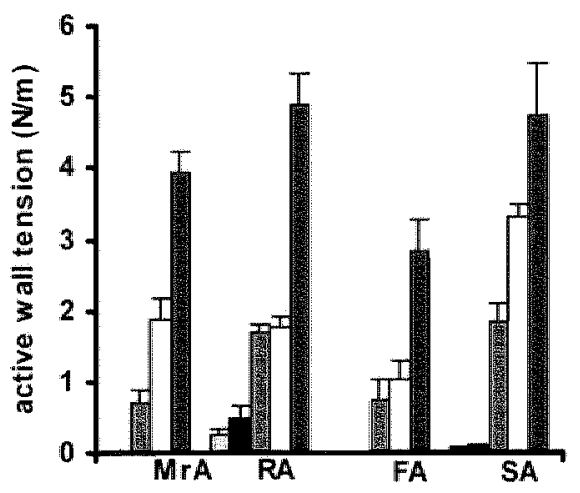

$\mathbf{B}$

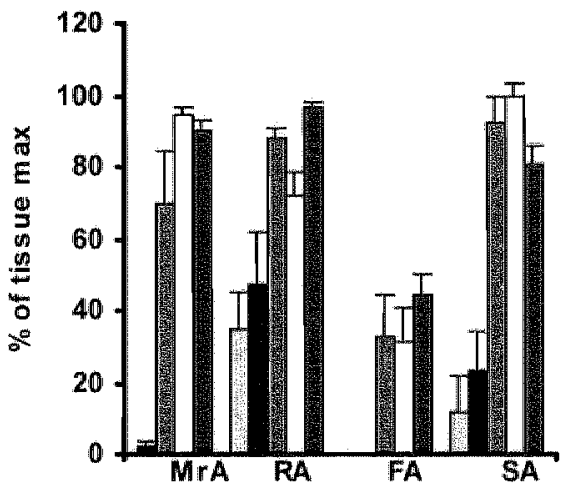

Figure 2.3. Maximal contractile responses to phenylephrine in absolute values (A) and expressed as \% of Tmax (B), in first order mesenteric resistance (MrA), renal (RA), femoral (FA) and saphenous arteries (SA). \pm S.E.M. 
Exposure of arterial segments to the $\alpha_{1}$-adrenergic agonist phenylephrine caused all vessels to contract. Contractile responses gradually increased with age and reached highest values at the age of 12 weeks (fig 2.3.a). Responses expressed as percentage of Tmax also showed an increased contractility during development. However, in the MrA and SA, contractile responses to $a_{1}$-adrenoceptor stimulation with phenylephrine at 10 days of age were smaller than those to electrical nerve stimulation. This relative increase of contractility mainly took place before the first 15 days of maturation and did not increase any further after this time point (fig. 2.3.b). Table 2.2 shows that sensitivity for phenylephrine also did not change after 15 days of postnatal development. Except in the femoral artery, where sensitivity decreased again in the adult animal. Contractile responses to phenylephrine of the femoral artery were larger than responses to sympathetic nerve stimulation, but they remained much smaller than in the other vessels.

Table 2.2. Sensitivity of rat mesenteric resistance, renal femoral and saphenous arteries to phenylephrine, isoproterenol and forskolin (pD2).

\begin{tabular}{lccccc}
\hline & Day 6 & Day 10 & Day 15 & Day 21 & Adult \\
\hline \multicolumn{1}{c}{ MrA } & & & & & \\
Norepinephrine & N.A. & $5.17 \pm 0.05$ & $5.31 \pm 0.04$ & $5.08 \pm 0.09$ & $5.24 \pm 0.13$ \\
Phenylephrine & N.A. & N.A. & $5.09 \pm 0.39$ & $5.63 \pm 0.05$ & $5.58 \pm 0.16$ \\
Isoproterenol & N.A. & $7.13 \pm 0.08$ & $7.44 \pm 0.24$ & $7.17 \pm 0.08$ & $6.97 \pm 0.12$ \\
Forskolin & N.A. & $7.33 \pm 0.58$ & $7.18 \pm 0.13$ & $6.47 \pm 0.06$ & $6.89 \pm 0.28$ \\
$\quad$ & & & & \\
RA & & & & & \\
Norepinephrine & $5.33 \pm 0.28$ & $5.62 \pm 0.07$ & $5.93 \pm 0.17$ & $6.10 \pm 0.31$ & $6.18 \pm 0.04$ \\
Phenylephrine & $5.63 \pm 0.34$ & $5.52 \pm 0.17$ & $6.31 \pm 0.28$ & $6.43 \pm 0.18$ & $6.10 \pm 0.09$ \\
Isoproterenol & $7.36 \pm 0.13$ & $7.17 \pm 0.06$ & $6.80 \pm 0.10$ & $6.93 \pm 0.11$ & $7.01 \pm 0.18$ \\
Forskolin & $7.34 \pm 0.12$ & $7.48 \pm 0.08$ & $7.07 \pm 0.21$ & $6.35 \pm 0.15$ & $6.02 \pm 0.09$ \\
$\quad$ FA & & & & & \\
Norepinephrine & To0 small & Too small & $5.37 \pm 0.01$ & $6.42 \pm 0.26$ & $5.41 \pm 0.03$ \\
Phenylephrine & Too small & Toosmall & $5.69 \pm 0.13$ & $5.56 \pm 0.18$ & $5.10 \pm 0.20$ \\
Isoproterenol & $7.19 \pm 0.19$ & $7.52 \pm 0.12$ & $7.29 \pm .10$ & $6.72 \pm 0.16$ & $6.78 \pm .13$ \\
Forskolin & $7.29 \pm 0.07$ & $7.42 \pm .13$ & $7.44 \pm 0.29$ & N.A. & $6.30 \pm 0.27$ \\
$\quad$ SA & & & & & \\
Norepinephrine & $5.67 \pm 0.52$ & $5.85 \pm 0.03$ & $5.47 \pm 0.09$ & $5.56 \pm 0.11$ & N.A. \\
Phenylephrine & $6.21 \pm 0.32$ & $5.70 \pm 0.25$ & $5.60 \pm 0.11$ & $5.62 \pm 0.11$ & $5.33 \pm 0.09$ \\
Isoproterenol & $7.40 \pm 0.23$ & $7.43 \pm 0.03$ & $7.09 \pm 0.15$ & $6.87 \pm 0.05$ & $6.71 \pm 0.16$ \\
Forskolin & $7.10 \pm 0.07$ & $7.25 \pm 0.00$ & $7.47 \pm 0.25$ & N.A. & $6.49 \pm 0.12$ \\
\hline
\end{tabular}

Sensitivity ( $\mathrm{pD2}=-\log$ EC50) is shown as mean \pm S.E.M. Values were not calculated when Emax $<0.3$ Tmax; N.A. not available. 
Neurogenic (capsaicin), peptidergic (CGRP) and adrenergic (isoproterenol) stimulation of adenylyl cyclase caused relaxation of all tested arteries. These relaxing responses progressively decreased during development, except in the mesenteric resistance artery.

In all the tested arteries $40 \mathrm{mmol} \mathrm{K}+$ caused contractile responses that remained stable for at least 20 minutes. Capsaicin transiently reduced the $\mathrm{K}+$-induced contraction. The relaxing effect of capsaicin faded after 1 to 5 minutes. In the continuous presence of capsaicin, the $\mathrm{K}+$-induced contraction ultimately stabilized between 10 and 20 minutes. Relaxing responses induced by the release of endogenous CGRP from sensory-motor nerves during administration of capsaicin gradually decreased during development in the renal and femoral arteries (fig. 2.4.a). After the age of 10 days the relaxing effect of exogenous applied CGRP gradually decreased with age, except in the mesenteric arteries (fig. 2.4.b).

A

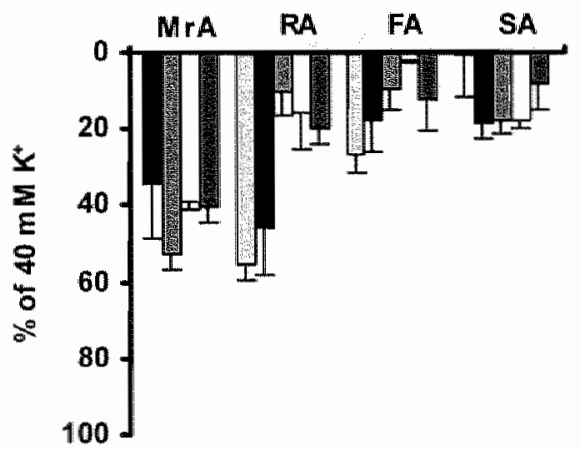

B

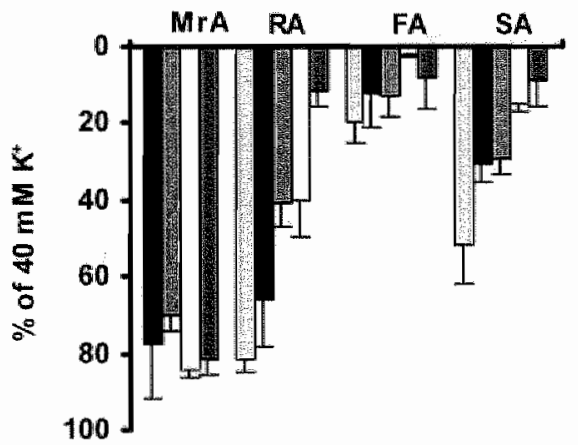

Figure 2.4. Maximal relaxing responses to endogenous CGRP (1 HM capsaicin) (A) and to exogenously applied CGRP (B), expressed as percentage relaxation of a precontraction induced by $40 \mathrm{mM} \mathrm{K}$, in first order mesenteric resistance (MrA), renal (RA) femorial (FA) and saphenous anteries (SA). day $6, \square$ day 10, day $15, \square$ day 21 , adult. Data are mean S. E.M. 
Figure 2.5 summarizes relaxing responses to the $\beta$-adrenergic agonist isoproterenol during contraction induced by potassium $(40 \mathrm{mM})$. In all types of arteries, isoproterenol caused concentration-dependent relaxation at the youngest age examined. Maximal relaxing responses to isoproterenol decreased during development in renal, femoral and saphenous arteries after the age of 10 days and are almost completely abolished in the renal artery at 12 weeks, while sensitivity for isoproterenol did not change during maturation (table 2.2). Only in the femoral artery, sensitivity to isoproterenol decreased after the age of 15 days.

Mesenteric resistance arteries showed a different developmental pattern of $\beta$-adrenergic responses. Maximal relaxation to isoproterenol did not change during the first 12 weeks of post-natal life. Because isoproterenol acts via Gs on adenylyl cyclase, which in turn induces cAMP dependent relaxation, we also evaluated relaxing responses to forskolin, which directly stimulates adenylyl cyclase. Decreased relaxing responses to isoproterenol during development (fig. 2.5), went together with a decreased sensitivity to forskolin (table 2.2).

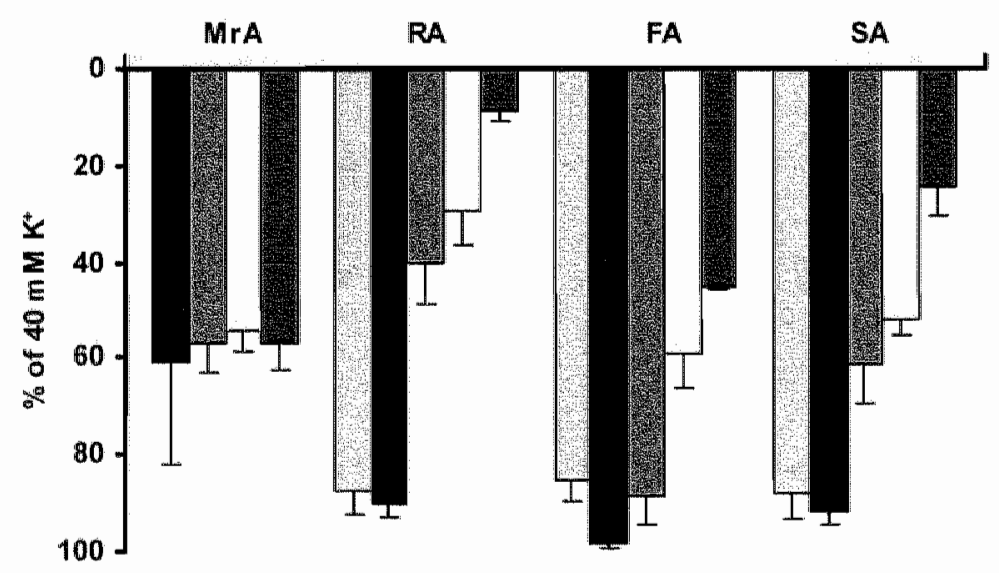

Figure 2.5. Maximal relaxing responses to $\beta$-adrenoceptor stimulation with isoproterenol expressed as percentage relaxation of a precontraction induced by $40 \mathrm{mM} \mathrm{K}$, in first order mesenteric resistance (MrA), renal (RA), femoral (FA) and saphenous arteries (SA). day 6 , day 10, , day $15, \square$ day 21 , adult. Data are mean \pm S.E.M. 


\section{Perivascular nerve density}

Staining of catecholamine- and CGRP-containing perivascular nevves shows that perivascular autonomic nerves are already present at the age of 6 days and after this nerve densities in the renal, femoral and saphenous arteries change only moderately (fig. 2.6 and 2.7). However, in mesenteric resistance arteries, autonomic nerve densities keep increasing during the first 12 weeks of post-natal development (fig. 2.6.a and 2.6.e, 2.7.a and 2.7.e)

Nerve densities differ markedly between vascular beds and these regional differences are similar for both types of autonomic nerves. A high density of catecholamine-containing nerves coincides with a high density of CGRPcontaining nerves. Especially mesenteric resistance (fig. 2.6.e and 2.7.e) and saphenous arteries (fig. $2.6 \mathrm{~h}$ and $2.7 \mathrm{~h}$ ) are very densely innervated, while renal (fig. 2.6.f and 2.7.f) and femoral arteries (fig. 2.6.g and 2.7.g) display a low density of autonomic nerves. Differences in nerve densities occur particularly along the femoral artery and at the branching point from the femoral to the saphenous artery. While the upper part of the femoral artery lacks nerves, the central part (half way between the iliac bifurcation and the saphenous artery) displays a plexus of low-density nerve fibers and at the transition from the femoral artery to the saphenous artery nerves densities increase dramatically (not shown).
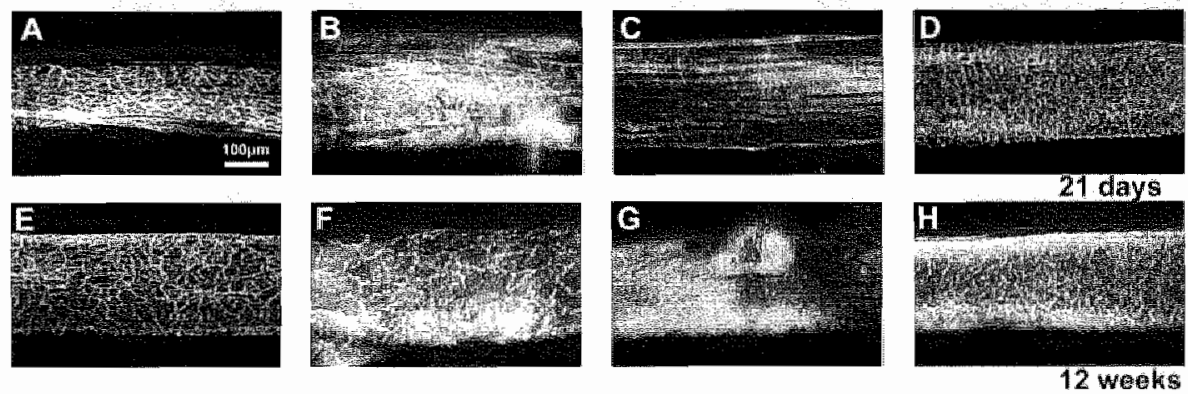

Figure 2.6. Stainings of catecholamine containing nerves showing different nerve densities between different arteries of 21 days and 12 weeks old animals. First order mesenteric resistance $(A$ and $E)$, renal $(B$ and $F)$, femoral $(C$ and $G)$ and saphenous arteries $(D$ and $H)$. 
A

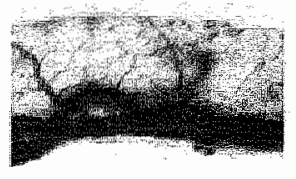

$1000 \mathrm{~min}$

E

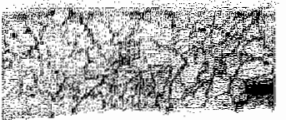

B

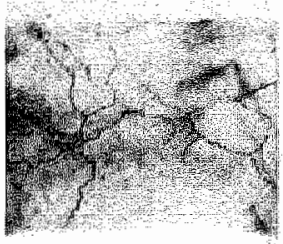

(

F

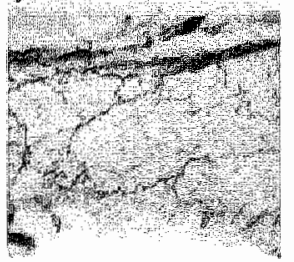

c

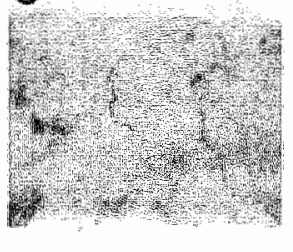

D

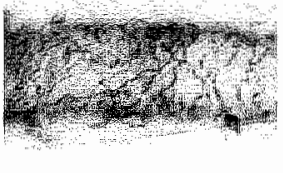

21 days

G

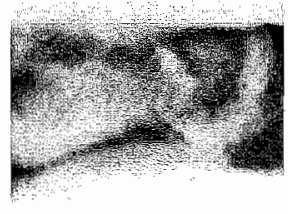

H

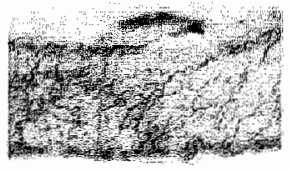

12 weeks

Figure 2.7. Sttainings of CGRP-containing nerves showing different nerve densities between different arteries of 21 days and 12 weeks old animals. First order mesenteric resistance ( $A$ and $E$ ), renal ( $B$ and $F$ ), femoral ( $C$ and $G$ ) and saphenous arteries ( $D$ and $H)$.

\section{Discussion}

During early postnatal development in the rat, the autonomic reactivity of peripheral arteries changes both quantitatively and qualitatively. The vessel wall and diameter grow considerably and this is accompanied by a marked increase in maximal contractile responses. Before the occurrence of responses to $\alpha_{1}$-adrenergic stimulation, a periarterial sympathetic innervation has already developed and all arteries were able to respond to nerve stimulation.

Despite structural growth, maximal active wall stress remains constant in the renal, femoral and saphenous arteries. These also show a progressive fading of relaxing effects of sensory motor nerve stimulation, exogenous CGRP and $\beta$-adrenoceptor activation, while CGRP-containing nerve densities do not change. This seems to be due to a decreased responsiveness to adenylyl cyclase activation. However, in mesenteric resistance arteries, adenylyl cyclase mediated responses do not decrease during the first 12 weeks of postnatal life and maximal active wall tension and autonomic nerve density keep increasing with age.

In rodents, the sympathetic nervous system is immature at birth and the development of autonomic arterial responsiveness occurs during the first 
weeks of postnatal life ${ }^{102,111}$, while in precocious animals some of these autonomic responses are already present in utero ${ }^{112,113}$.

In this study we evaluated postnatal development of arterial autonomic function in different peripheral arteries of the rat, with marked regional differences in autonomic nervous density.

Maximal arterial contractility increased 10 fold between 6 days and 12 weeks of age. Because maximal active wall stress remains constant in three of the four tested arteries, an extensive growth of the vessel wall is largely responsible for the increasing maximal contractility. The diameter increased 3-5 fold, while media cross sectional area increased 15 fold and media to lumen ratios doubled. The marked developmental growth of the arterial wall is probably due to an increased local blood flow and blood pressure, since the artery wall is very sensitive to these hemodynamic forces which regulate growth and remodeling of arteries during development ${ }^{19.22}$. It has been reported that neonatal sympathetic denervation results in a reduction of maximal contractile activity and thickness of the media in the adult ${ }^{97}$. However, we found that densely innervated mesenteric and saphenous arteries have similar developmental increases in media to lumen ratios as the poorly innervated renal and femoral arteries. This suggests that only minor neural input is required to mediate arterial smooth muscle hypertrophy and differentiation during development.

Both perivascular sympathetic and sensory-motor nerves were already present at 6 days of age. Densities of sympathetic and sensor motor nerves differed markedly between different parts of the arterial tree. For instance, while the upper region of the femoral artery lacks nerves, the central region (half way between the iliac bifurcation and the saphenous artery) displays a plexus of low-density sympathetic nerve fibers. Even more dramatic changes occur at branching points such as between upper superficial and deep femoral artery and saphenous artery (this study) and between superior mesenteric artery and its first order side branches ${ }^{114}$. These regional differences were similar for both types of autonomic nerves. A high density of CGRP-containing nerves coincides with a high density of catecholaminecontaining nerves. This seems in contrast with findings that long term neonatal sympathectomy was followed by an increased number of CGRP. containing nerves ${ }^{29}$. Moreover, experiments after long term neonatal denervation of sensory-motor nerves with capsaicin have shown that these nerves have a trophic influence on the development and function of sympathetic nerves ${ }^{30}$. The absence of sympathetic nerves might have increased the availability of nerve growth factor (NGF) for CGRP-containing nerves and vice versa. It has been described that a1-adrenoceptor stimulation inhibited the NGF-evoked stimulation of CGRP production and release from dorsal root ganglia neurons of adult rats ${ }^{115}$. These findings indicate that the presence of sensory-motor nerves is a prerequisite for an 
efficient development and function of sympathetic nerves. This in turn is necessary for maintaining optimal function of sensory-motor nerves and the development of adrenergic vascular function. Possibly, the presence of nerve growth factors during development differs extensively between different parts of the arterial system ${ }^{27,28}$, which would explain the differences in autonomic nerve densities between the vascular beds. Between 6 days and 12 weeks of age autonomic nervous densities changed only moderately, while during the same period arterial diameter increased 3-5 fold. Thus, to preserve nervous densities, a considerable growth of nerve fibers has to take place. Other factors that can affect arterial neurogenic control like density of varicosities and nerve endings and of the width of the synaptic cleft $^{98,116}$, were not addressed.

Femoral arteries showed minor responses to sympathetic nerve stimulation during the entire postnatal period tested. These small responses are in agreement with the low density of sympathetic nerves and the low sensitivity and small maximal contractile responses to $\alpha_{4}$-adrenergic agonist in this artery. In the mesenteric, renal and saphenous artery, responses to $\alpha_{1-}$ adrenoceptor activation and sympathetic nerve stimulation were quite strong. despite an unexpectedly low density of glyoxylic acid staining in the renal artery. This might be due to a rather high sensitivity of the renal artery to $\alpha_{1}$ adrenergic agonists. Vascular contractile responses to nerve stimulation and agonists might also be associated with the electrical coupling between innervated and non-innervated smooth muscle cells, receptor density and their subsequent second messenger system, like contractile proteins and their calcium- and phosphorylation-dependent and-independent control ${ }^{117}$.

We observed that coincidentally with the appearance of contractile responses to sympathetic nerve stimulation and $\alpha_{1}$-adrenoceptor activation, relaxing responses to adrenergic and neurogenic stimulators of adenylyl cyclase progressively decrease during maturation of renal, femoral and saphenous arteries, but not in the mesenteric resistance artery. Relaxing responses induced by the release of endogenous CGRP from sensory-motor nerves during administration of capsaicin decreased with age, while nerve densities of sensory-motor nerves did barely change during postnatal development. The observed reduction of post-junctional effects of CGRP seems to be responsible for the fading relaxing response to sensory-motor nerve stimulation. CGRP receptors on blood vessels are, beside other mechanisms, which we excluded in the present study (see materials and methods), linked to the activation of adenylyl cyclase ${ }^{118}$.

Also, arterial relaxing responses to $\beta$-adrenoceptor activation faded during postnatal development of these arteries. Stimulation of $\beta$-adrenoceptors activates adenylyl cyclase via $G_{s}$ proteins, which results in a cAMPdependent relaxation of the vessels. Activated $\beta$-adrenoceptors can activate both $G_{s}$, and the inhibitory $G$ protein, $G_{i}{ }^{119}$. 
Age-dependent decreases in $\beta$-adrenergic relaxation are coupled to large increases in $G_{i}$, but not $G_{0}$ or $G_{s}^{120}$. This could be due to a developmental increase in sympathetic activity. Neonatal sympathectomy has been observed to result in elevated levels of membrane bound $\beta$-adrenoceptors and an increased sensitivity of adenylyl cyclase stimulation by isoproterenol $^{121}$.

Our results showed a developmental reduction in sensitivity to direct stimulation of adenylyl cyclase by forskolin, while maximal relaxing responses did not change during maturation. This suggests that not only the coupling of the $\beta$-adrenergic and CGRP-receptor to stimulatory $G$ proteins is prevented or that there is an increased activity of the inhibitory $G$ proteins, but that also the subsequent signal transduction system is inhibited. In the adult animal the response to $\beta$-adrenoceptor stimulation is characterized by desensitization and receptor down-regulation to prevent excessive or prolonged excitation $^{122}$.

B-receptors are highly expressed, but poorly coupled to $G$ proteins in embryonic and early fetal development. However, during late gestation a functional receptor-Gs protein complex is established ${ }^{123}$ and a down regulation or desensitization of the $\beta$-adrenergic response could not be observed during this late period of fetal life ${ }^{124}$. The maintenance of $\beta$ adrenergic responses along with exposure to circulating and neurogenic catecholamines during birth, probably occurs to maintain an optimal supply of oxygen and nutrients to the heart and brain ${ }^{99,125}$. This might explain why there are still large arterial adenylyl cyclase mediated responses present during the early postnatal development.

It is also possible that arterial relaxing responses to adenylyl cyclase stimulation are, in contrast to $\alpha_{1}$-adrenoceptor mediated contractile responses, typical for immature and not fully differentiated arterial smooth muscle cells. In previous studies we found that relaxing responses to forskolin, which were absent in adult (20-wk-old) freshly isolated renal artery segments, reappeared after exposure to growth factors during arterial organ culture and were accompanied by a increased DNA synthesis ${ }^{12 B}$.

Adienylyl cyclase dependent responses in renal, femoral and saphenous arteries faded during postnatal development, while relaxing responses to (receptor mediated) activation of adenylyl cyclase did not change in mesenteric resistance arteries during the first 12 weeks of life. Additionally, maximal active wall tension and autonomic nerve density continued to increase in mesenteric resistance arteries, in contrast to the other tested vessels. The blood supply of the intestines might need different control mechanisms than other organs, because they are exposed to changes in nutritional intake between pre- and postnatal life. During prenatal life the fetus swallows amniotic fluid continuously, while during postnatal life feeding is based on intermittent loading of nutrients. 
In summary, arteries are innervated to a different extent during development and the maturation of adrenergic and peptidergic responses occur in a distinct way in different vascular beds. Ingrowing sympathetic and sensorymotor nerves may redistribute receptors on the cell surface or can be involved in the post receptor expression of G-proteins or the subsequent signal transduction system. However the exact nature of these interactions remain to be established, particularly since a sub optimal intra-uterine environment, due to fetal hypoxia and/or malnutrition, can change growth potential and arterial autonomic development ${ }^{3}$. This can have major consequences later in life, like an increased risk for cardiovascular diseases in the adult'. 


\section{Prenatal stress changes rat arterial adrenergic reactivity in a regionally selective manner}

Marijke W. Sanders, Gregorio E. Fazzi, Ger M. J. Janssen, Carlos E. Blanco, Jo G. R. De Mey

European Journal of Pharmacology. 2004; 488: 147-155 


\begin{abstract}
A suboptimal fetal environment has been linked to increased risk of cardiovascular disease in adulthood. We investigated whether intrauterine stress (IUS) alters the development of adrenergic reactivity in different types of rat arteries. Intrauterine stress was induced by ligation of the uterine arteries at day 13 of pregnancy in Wistar rats. First order mesenteric, renal, femoral and saphenous arteries of the 21-day-old male offspring were studied in a myograph. Intrauterine stress in the rat changes arterial adrenergic reactivity in a regionally selective manner. Adrenoceptor mediated responses are altered in the renal artery. Maximal contractile responses to phenylephrine were increased, while sensitivity to the $a_{1}$ adrenoceptor agonist was decreased. intrauterine stress significantly reduced contractile responses to norepinephrine and enhanced relaxing responses to isoproterenol in the renal artery. Adrenergic responses were not modified in mesenteric, femoral and saphenous arteries. In the kidneys the densities of $\left[{ }^{3} \mathrm{H}\right]$ prazosin binding sites, periarterial adrenergic nerves and of the glomeruli were not altered after intrauterine stress at day 13 of gestation. The observed regionally selective alterations in arterial reactivity might link a suboptimal fetal environment to the development of cardiovascular disease in the adult.
\end{abstract}




\section{Prenatal stress changes rat arterial adrenergic reactivity in a regionally selective manner}

\section{Introduction}

Cardiovascular disease is associated with genetic predisposition, smoking, obesity, lack of exercise and diabetes mellitus. In addition, there is now substantial evidence that adverse influences during fetal life increase the susceptibility to cardiovascular, endocrine and metabolic diseases later in life $^{1}$. A suboptimal supply of oxygen ${ }^{3,4}$ and nutrients caused by maternal undernutrition $^{2}$ or by disturbed placental function ${ }^{127}$ alter the fetal development of cardiovascular control mechanisms. The exact mechanisms that link a suboptimal fetal environment to the programming of adult disease are unknown. A number of neuro-humoral systems and organs, like the renin-angiotensin system, the hypothalamic-pituitary-adrenal axis ${ }^{7}$, vascular endothelial function and sympathetic innervation ${ }^{3,6.8}$ and the kidneys ${ }^{9}$ have been proposed to be involved in what is increasingly recognized as signs of the epigenetics of chronic disease.

Sympathetic nerves innervate the vascular tree, but there are considerable differences in the density and regulatory actions of the sympathetic nervous fibres between different vascular beds. Adrenoceptors mediate the vascular actions of the sympathetic nervous system. The most important neurotransmitter, norepinephrine, can bind to $\alpha_{1^{-}}, \alpha_{2^{-}}$and $\beta$-adrenoceptors which cause vessels to contract or relax ${ }^{128,129}$. Hyperinnervation of the cardiovascular system precedes the structural and functional changes of the 
vasculature in spontaneously hypertensive rats ${ }^{11: 12}$ and eventually leads to an increased peripheral resistance, cardiovascular hypertrophy and high blood pressure.

Studies in our own laboratory have demonstrated that a suboptimal fetal environment can cause sympathetic hyperinnervation of the peripheral arterial system ${ }^{3}$. Since sympathetic nerves, neurotransmitter release and the subsequent stimulation of different receptor subtypes are important in maintaining normal cardiovascular dynamics and vascular structure, we hypothesize that a suboptimal fetal environment can change normal development of arterial adrenergic function.

In this study, we induced an experimental intrauterine stress (IUS) by bilateral ligation of the uterine arteries at day 13 of pregnancy in Wistar rats and investigated the consequences of intrauterine stress for adrenergic vascular responses in the offspring. To evaluate whether intrauterine stress affects regionally different vascular beds in a different manner, mesenteric resistance, renal, femoral and saphenous arteries were taken from the 21 day old offspring and studied with pharmacological methods. Changes in pattern and density of cathecholamine-containing nerves were also assessed using fluorescence immunohistochemistry and $\alpha_{1}$-adrenoceptors were approached by radialigand binding.

\section{Materials and methods}

\section{Animals}

Experiments were approved by the local ethical committee for animal research of the University of Maastricht (registration number 2000-68). Animals had free access to pelleted food and tap water and were maintained on a 12 -h light/dark cycle at $21^{\circ} \mathrm{C}$.

\section{Induction of intrauterine stress}

Female Wistar rats (Charles River, Maastricht, The Netherlands) weighing 200-250g were mated. Day 1 of pregnancy was defined as the day immediately following the night during which males were present and conception was confirmed by the vaginal smear method. To induce intrauterine stress, on day 13 and day 17 of pregnancy, animals were subjected to bilateral ligation of the uterine arteries (near the iliac arteries), according to the method of Wigglesworth ${ }^{5}$. A sham procedure was performed as described, except for the actual occlusion of the blood vessels. The offspring of mothers, which underwent bilateral ligation of the uterine arteries during pregnancy, are referred to as IUS (intrauterine stress)-group and the offspring of mothers, which underwent a sham operation, are 
referred to as control group. Rats delivered spontaneously at 22 days and litter size and birth weight of the offspring were determined within one hour after birth.

Because the number of life-born pups was reduced after intrauterine stress, litter sizes of the control and intrauterine stress group were matched within one hour after birth. At 21 days after delivery, animals were killed by cervical dislocation.

\section{Arterial reactivity}

Arterial segments were isolated from the 21-day-old male offspring of both groups. Two stainless-steel wires (diameter $40 \mu \mathrm{m}$ ) were inserted in the lumen of the arterial segments, which were then mounted in organ chambers between an isometric force transducer and a displacement device (Danish Myotechnology by J.P. Trading, Denmark). The organ chambers were filled with Krebs-Ringer bicarbonate solution which was maintained at $37^{\circ} \mathrm{C}$ and continuously aerated with $95 \% \quad \mathrm{O}_{2}$ and $5 \% \quad \mathrm{CO}_{2}$. Before the actual experiments started, arterial segments were stretched to their individual optimal lumen diameter for mechanical performance, i.e. the diameter at which maximal contractile responses $\mathrm{K}^{+}(40 \mathrm{mM})$ were obtained ${ }^{103}$. In most experiments a first order mesenteric resistance, right renal, right femoral and right saphenous arterial segment from one animal were mounted in an organ chamber and studied in parallel. At the start of the experiments, all arterial preparations were exposed to $1 \mu \mathrm{M}$ capsaicin during $20 \mathrm{~min}$. to stimulate and permanently desensitise sensory-motor nerves, while it does not affect efferent fibres of the autonomic nervous system ${ }^{107,108}$.

Concentration dependent $(0.01 \mu \mathrm{M}$ to $10 \mu \mathrm{M})$ contractile responses to phenylephrine, norepinephrine, 5-hydroxytryptamine and electrical field stimulation were expressed as active wall tension $(\mathrm{N} / \mathrm{m})$ or as percentage of the contraction, induced by a mixture of $125 \mathrm{mM} \mathrm{K} \mathrm{K}^{+}$and $10 \mu \mathrm{M}$ phenylephrine. This latter response which we refer to as maximal tissue contraction (Tmax), results from strong activation of electro- and pharmacomechanical coupling by $\mathrm{K}^{+}$and phenylephrine, respectively. Sympathetic neuroeffector mechanisms were studied using electrical field stimulation (EFS; $0.25-32 \mathrm{~Hz}, 2 \mathrm{msec}, 85 \mathrm{~mA}$ ) via platinum electrodes which were placed in the axial direction over the vessel segments, and a stimulator designed by the local technical department.

Concentration dependent $(0.01 \mu \mathrm{M}$ to $10 \mu \mathrm{M})$ relaxing responses to isoproterenol, calcitonin gene-related peptide (CGRP) and forskolin were investigated during contraction induced by $40 \mathrm{mM} \mathrm{K}^{+}$to blunt effects of the tested compounds on sarcolemmal $\mathrm{K}^{4}$-channels ${ }^{109}$, in order to concentrate on ultimate stimulatory influences on adenylyl cyclase. Relaxing responses were expressed as percentage change of the pre-existing contractile tension. Effects of isoproterenol on $\alpha_{4}$-adrenoceptors were prevented by 1 
LM prazosin. After experimentation; arteries were fixed overnight at their optimal diameter in phosphate-buffered $(\mathrm{pH} 7.4)$ formaldehyde $(4 \%)$.

\section{Morphometric analysis of the arteries and their adrenergic nerve fibres}

Structural properties of first order mesenteric resistance, renal, femoral and saphenous arteries were determined according to the morphometric methods described by Stassen et al. ${ }^{110}$. Glyoxylic acid was used to visualize and quantify periarterial adrenergic nerve fibres in whole mount preparations ${ }^{3}$.

\section{Morphometric analysis of the kidneys}

Left kidneys were dissected, fixed overnight in phosphate-buffered ( $\mathrm{pH} 7.4$ ) formaldehyde ( $4 \%$ ) and embedded in paraffin. Parallel transversal sections were stained with Jones methenamine silver. Number, area and circumference of the glomeruli were measured using video images generated by a Zeiss Axioscope (Zeiss, Germany), a standard CCD camera (Sony) and commercially available software (Sigmascan Pro 2.0, Jandel Scientific, Erkrath, Germany). The number of glomeruli within a known volume was calculated according to the formula: $n=G / F \times A \times(D+T)^{130}$, with $\mathrm{n}$, density of glomeruli $\left(/ \mathrm{mm}^{3}\right) ; \mathrm{G}$, number of glomeruli counted in 10 fields of the kidney; $F$, number of fields counted; $A$, area $\left(1200356 \mu \mathrm{m}^{2}\right) ; D$, average diameter of the glomeruli $(62 \mu \mathrm{m}) ; \mathrm{T}$, section thickness $(4 \mu \mathrm{m})$. The glomeruli counted were localized in the cortical area immediately under the capsule.

\section{$a_{1}$-adrenoceptor binding assay}

Radioligand binding was used to determine the characteristics of $\alpha_{1}$ adrenoceptors in right kidney microsomes. Analysis of $\left[{ }^{3} \mathrm{H}\right] \mathrm{prazosin}$ binding was performed essentially as described by Michel et al. ${ }^{131}$.

\section{Drugs and solutions}

Krebs-Ringer bicarbonate buffer contained (in $\mathrm{mM}$ ): $\mathrm{NaCl} 118.5, \mathrm{MgSO}_{4} \cdot 7$ $\mathrm{H}_{2} \mathrm{O} 1.2, \mathrm{KH}_{2} \mathrm{PO}_{4} 1.2, \mathrm{NaHCO}_{3} 25.0, \mathrm{KCl} 4.7, \mathrm{CaCl}_{2} 2.5$ and glucose 5.5. A $40 \mathrm{mM} \mathrm{K}^{+}$solution was prepared by replacing part of the $\mathrm{NaCl}$ by an equimolar amount of $\mathrm{KCl}$. Phosphate-buffered solution consisted of $0.1 \mathrm{M}$, $\mathrm{NaH}_{2} \mathrm{PO}_{4} \cdot \mathrm{H}_{2} \mathrm{O}$ and $0.1 \mathrm{M} \mathrm{Na}_{2} \mathrm{HPO}_{4} \cdot 2 \mathrm{H}_{2} \mathrm{O}$. Calcitonin gene-related peptide (CGRP) was obtained from BACHEM (Bubendorf, Switzerland). Capsaicin, phenylephrine, 5-hydroxytryptamine, norepinephrine and prazosin were obtained from Sigma chemical co (St. Louis, MO, USA), isoproterenol and forskolin from ICN Pharmaceuticals (Costa Mesa, CA, USA) and Lawson solution from Boom b.v. (Meppel, The Netherlands). Phenylephrine, 5hydroxytryptamine, norepinephrine, isoproterenol and CGRP were dissolved in distilled water and capsaicin, prazosin and forskolin in ethanol $(100 \%)$. 


\section{Data analysis}

Concentration response curves were analysed in terms of sensitivity $\left(\mathrm{PD}_{2}=\right.$ logEC50) and maximal response (Emax) by fitting individual concentrationresponse data to a sigmoid regression curve and interpolation (Graphpad Prism version 2.01, Graphpad Software Inc.). Differences between findings in arteries from both groups of rats were tested with Student $t$ test or MannWhitney $U$ test when normality test (Kolmogorov-Smirnov) failed. A value of $P<0.05$ was considered statistically significant. Data are presented as mean \pm S.E.M.

\section{Results}

After bilateral ligation of the uterine arteries at day 13 of pregnancy, (1) fetal survival was reduced, as indicated by the small litter size, (2) birth weights were increased (table 3.1) and (3) absolute and relative organ weights were unchanged at 21 days of age (data not shown). The induction of intrauterine stress at a later time point of pregnancy (day 17) resulted in significantly decreased birth weights (table 3.1).

Table 3.1. Birth weight and litter size of the offspring at birth.

\begin{tabular}{lcccc}
\hline & \multicolumn{2}{c}{ Ligation at day 13 } & \multicolumn{2}{c}{ Ligation at day 17 } \\
\cline { 2 - 5 } & CON & IUS & CON & IUS \\
\hline Birth weight & $5.44 \pm 0.08$ & $6.04 \pm 0.10^{\$}$ & $5.35 \pm 0.04$ & $5.03 \pm 0.05^{8}$ \\
& $(n=84)$ & $(n=38)$ & $(n=204)$ & $(n=137)$ \\
Litter size & $8 \pm 1$ & $4 \pm 1^{\$}$ & $11 \pm 1$ & $5 \pm 1^{\$}$ \\
& $(n=14)$ & $(n=13)$ & $(n=16)$ & $(n=22)$ \\
\hline
\end{tabular}

IUS, intrauterine stress. Values are mean $\pm S . E . M_{\text {; }}{ }^{5} P<0.001$ versus control.

Optimal diameter and cross-sectional area of the media were larger in the renal and femoral arteries than in the mesenteric resistance and saphenous arteries. These arterial structural properties at 21 days of age were not modified by intrauterine stress (table 3.2 ). 
Table 3.2. Structural properties of first order mesenteric resistance, renal, femoral and saphenous arteries of 21 day-old-rats.

\begin{tabular}{ccccc}
\hline & \multicolumn{2}{c}{ CON } & \multicolumn{2}{c}{ WUS } \\
\cline { 2 - 5 } & $\begin{array}{c}\text { Diameter } \\
(\mu \mathrm{m})\end{array}$ & $\begin{array}{c}\text { MCSA } \\
\left(\times 1000 \mu \mathrm{m}^{2}\right)\end{array}$ & Diameter $(\mu \mathrm{m})$ & $\begin{array}{c}\text { MCSA } \\
\left(\times 1000 \mu \mathrm{m}^{2}\right)\end{array}$ \\
\hline MrA & $194 \pm 6$ & $\begin{array}{c}5.2 \pm 0.4 \\
(n=8)\end{array}$ & $197 \pm 10$ & $5.1 \pm 0.6$ \\
& & $21.2 \pm 1.8$ & $375 \pm 26$ & $(n=7)$ \\
RA & $373 \pm 13$ & $(n=16)$ & & $21.9 \pm 2.2$ \\
FA & $440 \pm 10$ & $33.9 \pm 2.1$ & $475 \pm 14$ & $(n=11)$ \\
& & $(n=9)$ & & $36.1 \pm 1.7$ \\
SA & $304 \pm 6$ & $16.2 \pm 1.1$ & $320 \pm 10$ & $(n=8)$ \\
& & $(n=9)$ & & $16.6 \pm 0.7$ \\
\end{tabular}

MCSA, media cross-sectional area. Values are mean \pm S.E.M.

\section{Arterial reactivity}

Maximal contractile responses (Tmax) to a mixture of $125 \mathrm{mM} \mathrm{K}^{+}$and $10 \mu \mathrm{M}$ phenylephrine were comparable for the four types of arteries, despite differences in diameter and media mass. Tmax was unchanged after intrauterine stress (fig. 3.1).

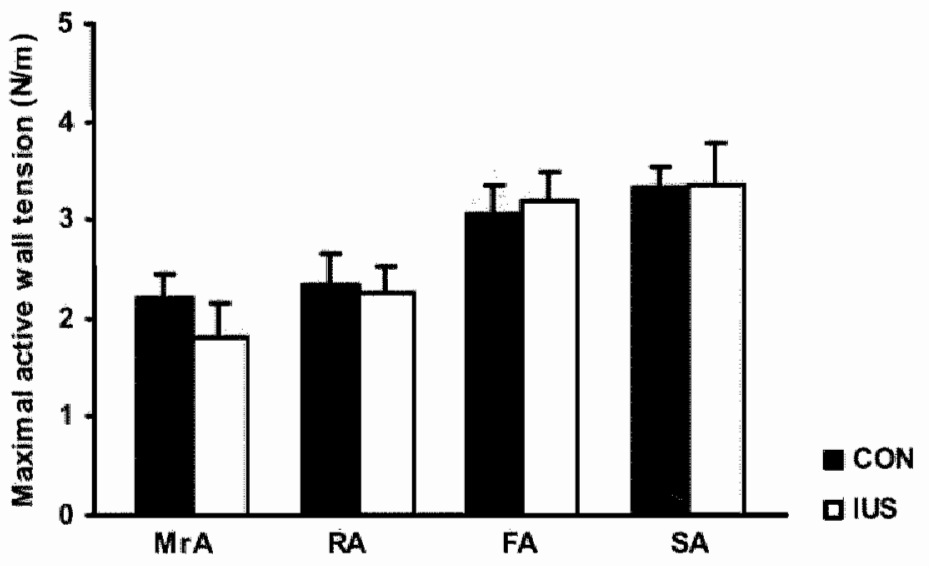

Figure 3.1. Maximal contractile responses (Tmax) to $125 \mathrm{mM} \mathrm{K}$ plus $10 \mu \mathrm{M}$ phenylephrine in first order mesenteric resistance (MrA), renal (RA), femoral (FA) and saphenous arteries (SA) of control rats $(\square, C O N)$ and rats that survived intrauterine stress $(\square$, UUS). Data are mean \pm S.E.M. 
All arterial preparations contracted in response to the $\alpha_{4}$-adrenoceptor agonist phenylephrine. Responses of the mesenteric resistance and saphenous arteries were considerably larger than those of renal and femoral arteries of control animals (fig. 3.2). Following intrauterine stress, maximal contractile responses to phenylephrine were significantly increased in the renal artery and reached a comparable reactivity as the mesenteric resistance and saphenous arteries (fig. 3.2). In addition to an increased maximal response, sensitivity to phenylephrine was significantly decreased in the renal artery after intrauterine stress (table 3.3). Intrauterine stress did not modify sensitivity and maximal responsiveness to the $\alpha_{y}$-adrenoceptor agonist in the mesenteric resistance, femoral and saphenous arteries (fig. 3.2).

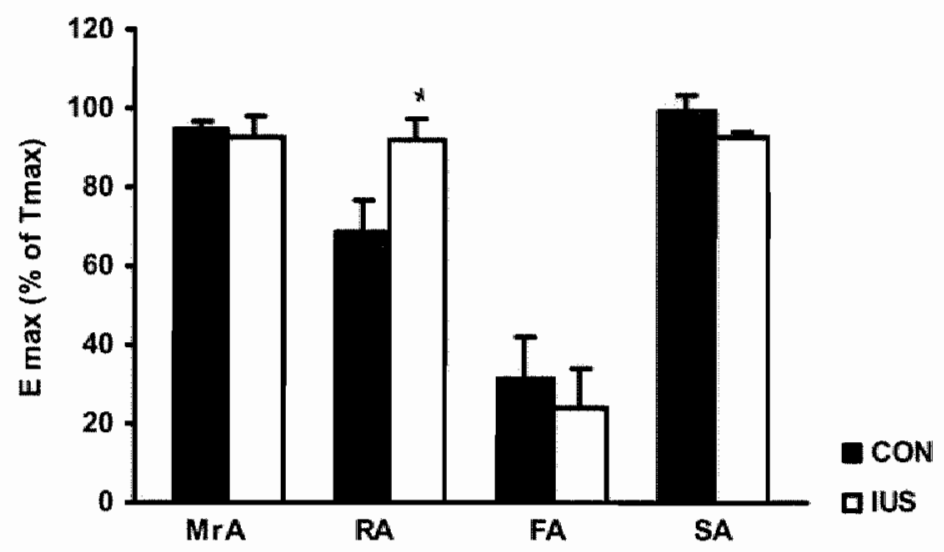

Figure 3.2. Maximal contractile responses (\% of Tmax) to phenylephrine in first order mesenteric resistance (MrA), renal (RA), femoral (FA) and saphenous arteries (SA) of control rats $(\square, C O N)$ and rats that survived intrauterine stress (L., IUS). Data are mean \pm S.E.M. " $P<0.05$ versus control.

To investigate whether intrauterine stress changes contractile responses to other agonists, we exposed renal arteries of control animals and of animals that survived intrauterine stress to increasing concentrations of 5hydroxytriptamine and vasopressin. Maximal responses to 5hydroxytriptamine were significantly increased after intrauterine stress and maximal responses to vasopressin seemed to be increased as well, but the differences were not statistically significant (table 3.3). Sensitivities to 5hydroxytriptamine and vasopressin were not altered after intrauterine stress (table 3.3). 
Table 3.3. Maximal contractile responses and sensitivity to vasoconstrictor and vasodilator stimuli in renal arteries at 21 days of age.

\begin{tabular}{|c|c|c|c|}
\hline & & CON & IUS \\
\hline \multirow{2}{*}{ 5-Hydroxytryptamine } & $E \max (\% \mathrm{Tmax})$ & $85.81 \pm 4.04$ & $99.82 \pm 4.52$ * \\
\hline & $\mathrm{pD}_{2}$ & $6.09 \pm 0.15$ & $6.01 \pm 0.10$ \\
\hline \multirow[t]{2}{*}{ Vasopressin } & $E \max \left(\% T_{\max }\right)$ & $76.92 \pm 8.13$ & $92.10 \pm 7.87$ \\
\hline & $\mathrm{pD}_{\mathrm{z}}$ & $6.69 \pm 0.10$ & $6.59 \pm 0.19$ \\
\hline \multirow{2}{*}{ Phenylephrine } & $E \max \left(\% T_{\text {max }}\right)$ & $68.56 \pm 7.84$ & $91.96 \pm 5.11^{*}$ \\
\hline & $\mathrm{pD}_{2}$ & $6.24 \pm 0.115$ & $5.61 \pm 0.11^{\text {i* }}$ \\
\hline \multirow[t]{2}{*}{ Norepinephrine } & $E \max (\% \operatorname{Tmax})$ & $97.15 \pm 3.34$ & $70.75 \pm 4.35$ * \\
\hline & $\mathrm{pD}_{2}$ & $6.10 \pm 0.31$ & $6.00 \pm 0.36$ \\
\hline \multirow{2}{*}{$\begin{array}{l}\text { Electrical Field } \\
\text { Stimulation }\end{array}$} & $\operatorname{Emax}\left(\% T_{\text {Tmax }}\right)$ & $23.02 \pm 5.89$ & $16.78 \pm 4.92$ \\
\hline & $E F_{s o}(H z)$ & $16.13 \pm 0.32$ & $16.96 \pm 0.46$ \\
\hline \multirow[t]{2}{*}{ |soproterenol } & Emax $\left(\% \mathrm{~K}^{+} 40 \mathrm{mM}\right)$ & $-26.67 \pm 10.78$ & $-57.55 \pm 7.31^{*}$ \\
\hline & $\mathrm{pD}_{z}$ & $6.89 \pm 0.17$ & $7.03 \pm 0.07$ \\
\hline
\end{tabular}

E max, maximal response. Tmax, maximal tissue contraction, induced by a mixture of $125 \mathrm{mM} \mathrm{K}^{*}$ and $10 \mu \mathrm{M}$ phenylephrine. $\mathrm{EF}_{50}$, frequency required for $50 \%$ of maximal response. Values are mean t S.E.M.; " $P<0.05$ versus control, ** $P<0.001$ versus control

Figure 3.3 summarizes maximal contractile responses to exogenous norepinephrine. Mesenteric resistance, renal and saphenous arteries contracted to the same extent, but the femoral artery barely responded to stimulation with norepinephrine. Intrauterine stress significantlly decreased maximal contractile responses to norepinephrine in the renal artery, while sensitivity was not modified (table 3.3). Intrauterine stress did not change the responses to norepinephrine in the other types of artery (fig. 3.3). 


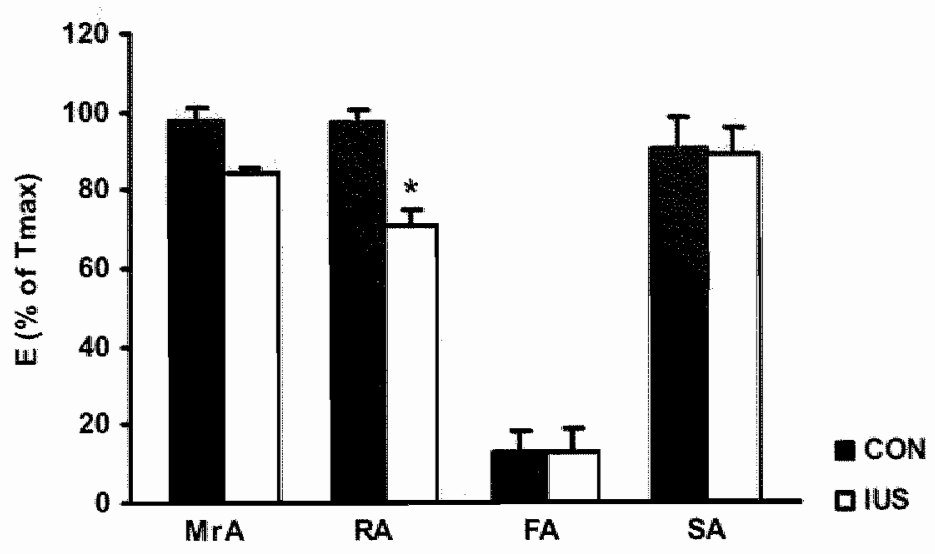

Figure 3.3. Maximal contractile responses (\% of Tmax) to norepinephrine in first order mesenteric resistance (MrA), renal (RA), femoral (FA) and saphenous arteries (SA) of control

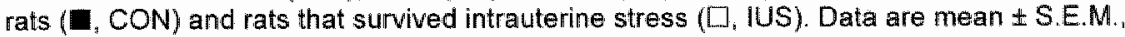
* $P<0.05$ versus control.

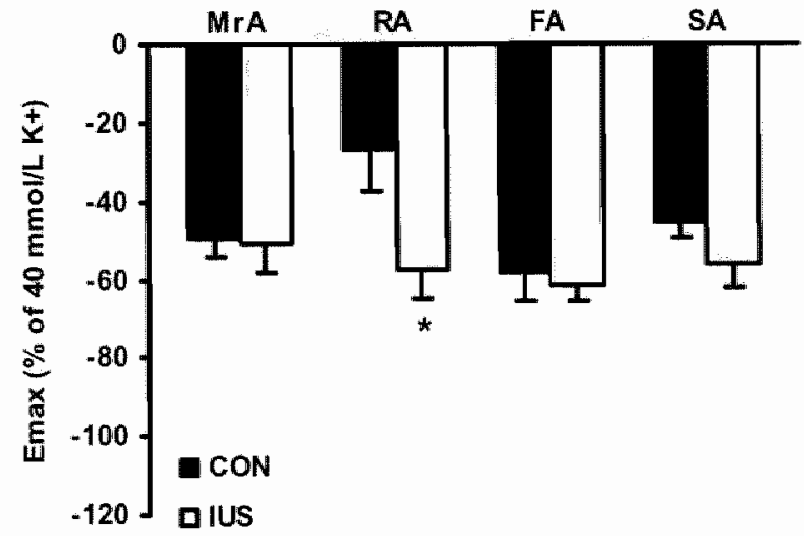

Figure 3.4. Maximal relaxing responses ( $\%$ of $40 \mathrm{mM} \mathrm{K}$ ) to isoproterenol in first order mesenteric resistance (MrA), renal (RA), femoral (FA) and saphenous arteries (SA) of control rats ( $\mathbf{E}$, CON) and rats that survived intrauterine stress ([.), IUS). Data are mean \pm S.E.M. ${ }^{*} \mathrm{P}<0.05$ versus control. 
Stimulation of $\beta$-adrenoceptors with isoproterenol, during precontraction with $40 \mathrm{mM} \mathrm{K}$, caused all vessels to relax. The maximal relaxing responses were similar in the mesenteric resistance, femoral and saphenous arteries, while the renal artery displayed smaller relaxing responses to isoproterenol. When the animals had suffered intrauterine stress, renal arterial relaxing responses to $\beta$-adrenoceptor stimulation were significantly increased compared to the control animals (fig. 3.4). Sensitivity to isoproterenol was unchanged in the renal artery after intrauterine stress (table 3.3). Intrauterine stress did not modify $\beta$-adrenergic relaxation in mesenteric resistance, femoral and saphenous arteries (fig. 3.4). Also when intrauterine stress was induced at day 17 of pregnancy, which decreased birth weight (table 3.1), renal arterial relaxing responsiveness to isoproterenol was enhanced $(-12.04 \pm 5.59 \%$ vs. $-51.64 \pm 11.30 \%, P=0.016$ )

Isoproterenol causes relaxation via stimulation of adenyly| cyclase. Consequently, we evaluated responses to direct activation of adenylyl cyclase by forskolin $(0.01 \mu \mathrm{M}$ to $10 \mu \mathrm{M})$. All vessels fully relaxed during contraction with $40 \mathrm{mM} \mathrm{K}^{+}$. Intrauterine stress did not alter maximal relaxing responses to forskolin, nor did it change sensitivity to the diterpene in either type of artery that we investigated (data not shown).

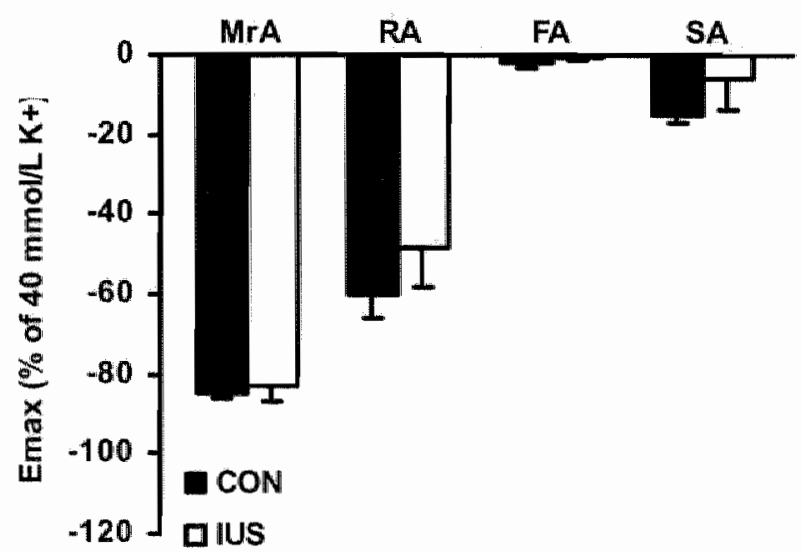

Figure 3.5. Maximal relaxing responses ( $\%$ of $40 \mathrm{mM} \mathrm{K}$ ) to CGRP in first order mesenteric resistance (MIFA), renal (RA), femoral (FA) and saphenous arteries ( $\mathrm{SA}$ ) of control rats (,$C O N)$ and rats that survived intrauterine stress (D., IUS). Data are mean \pm S.E.M. 
Another agonist that acts via adenylyl cyclase, CGRP $(0.1 \mu \mathrm{M})$, induced a considerable relaxation in the mesenteric resistance and renal artery, but hardly in the femoral and saphenous artery. Intrauterine stress did not modify relaxing responses to CGRP in either type of artery (fig. 3.5).

Maximal contractile responses to sympathetic nerve stimulation (electrical field stimulation after exposure to capsaicin) of the renal and femoral artery were smaller than in the mesenteric resistance and saphenous artery (fig. 3.6). Despite altered responses to $\alpha_{1}$ - and $\beta$-adrenoceptor agonists in the renal artery after intrauterine stress, contractile responses to electrical field stimulation were unchanged after surviving a suboptimal fetal environment (fig. 3.6). Neither did intrauterine stress alter neurogenic vasoconstriction in mesenteric resistance, femoral and saphenous arteries (fig. 3.6).

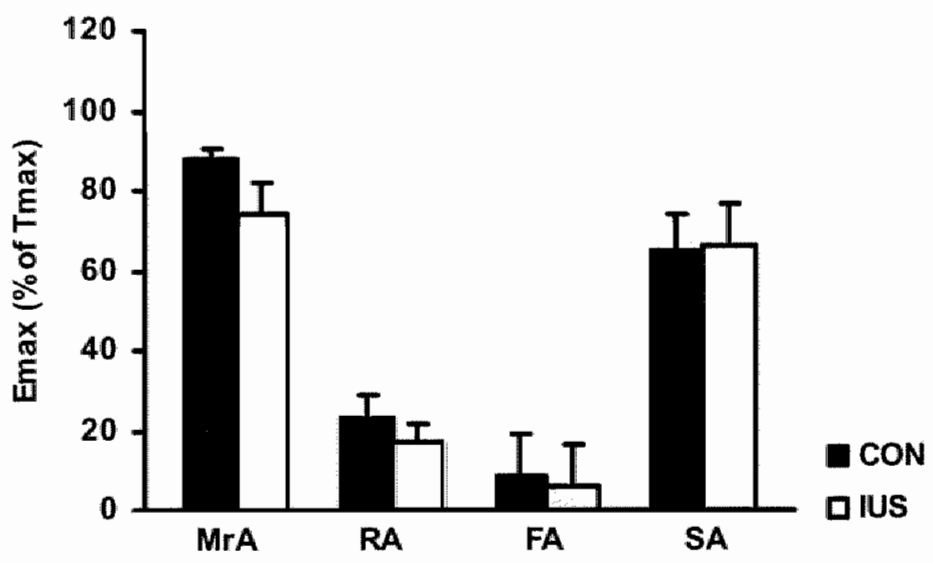

Figure 3.6. Maximal contractile responses ( $\%$ of Tmax) to electrical field stimulation (EFS) in first order mesenteric resistance (MrA), renal (RA), femoral (FA) and saphenous arteries (SA) of control rats $(\mathbf{C}$, CON) and rats that survived intrauterine stress (U, IUS). Data are mean 2 S.E.M.

\section{Ligand binding in kidney}

To gain information about $a_{1}$-adrenoceptors that might be indirectly relevant for renal arteries, we determined the affinity (Kd) and density (Bmax) of $\left[{ }^{3} \mathrm{H}\right]$ prazosin binding sites in kidney microsomes of control animals and of animals that suffered intrauterine stress. Although the affinity for $a_{1}$ adrenoceptors seems lower in the kidneys of intrauterine stress animals, these differences did not reach statistical significance ( $\mathrm{Kd}, 0.07 \pm 0.02 \mathrm{vs}$. $0.09 \pm 0.01 \mathrm{nM})$. The Bmax values calculated from saturation binding curves were comparable in kidneys of control and intrauterine stress animals (Bmax, $67.1 \pm 6.9$ vs. $58.0 \pm 7.4 \mathrm{fmol} / \mathrm{mg}$ protein). 


\section{Morphometry of the kidneys}

Our results in table 3.4 demonstrate that the density of glomeruli is not significantly modified in 21-day-old rats that have suffered intrauterine stress. Moreover, the area and the circumference of the glomeruli are not significantly altered after intrauterine stress.

Table 3.4. Morphology of kidneys of 21-day-old animals.

\begin{tabular}{lcc}
\hline & CON & IUS \\
\hline Kidney weight $(g)$ & $0.28 \pm 0.02$ & $0.30 \pm 0.01$ \\
Density of glomeruli $\left(\left\langle\mathrm{mm}^{3}\right)\right.$ & $373 \pm 36$ & $429 \pm 37$ \\
Area of glomeruli $\left(\mu \mathrm{m}^{2}\right)$ & $2699 \pm 202$ & $2495 \pm 167$ \\
Circumference of glomeruli $(\mu \mathrm{m})$ & $210 \pm 18$ & $190 \pm 6$ \\
\hline
\end{tabular}

Values are mean \pm S.E.M.

\section{Nerve density}

Adrenergic nerve fibre density differs markedly between vascular beds of the rat. Mesenteric resistance and saphenous arteries display a dense innervation, while renal and femoral arteries display only few nerve fibres. Staining of catecholamine-containing nerves with glyoxylic acid indicated that adrenergic fibre density was not modified in either type of artery of 21 day-old rats that survived intrauterine stress induced at 13 days of gestation (data not shown).

\section{Discussion}

Epidemiological findings suggest that an unfavourable fetal environment, resulting in low birth weight, predisposes individuals to develop chronic cardiovascular and metabolic diseases in adult life ${ }^{1,132,133}$. In the present study, we investigated whether experimental intrauterine stress in the rat alters the development of adrenergic reactivity in arteries from regionally different parts of the vascular tree, which play a major role in the control of total peripheral resistance and blood pressure.

The main finding of our study is that intrauterine stress resulted in a regionally selective alteration of arterial adrenergic function at three weeks after birth. While mesenteric resistance, femoral and saphenous arteries 
were not affected, renal arteries displayed selective pharmacological alterations.

Intrauterine stress induced at day 13 of pregnancy resulted in a marked reduction of the number of fetuses and an increased birth weight. Intrauterine stress induced at a later stage of gestation (day 17) reduced birth weight, as previously reported ${ }^{6,134}$. Thus, birth weight alone seems not to be an ideal index of the exposure of the fetus to an environment that leads to persistent cardiovascular alterations ${ }^{2,127}$. We recently reached a similar conclusion during a comparison of the effects of protein malnutrition and chronic moderate hypoxia on arterial endothelium-dependent reactivity in the chicken embryo ${ }^{70}$.

In the four types of arteries that we studied, maximal contractile responses were comparable, despite a larger optimal diameter and cross-sectional area of the media in renal and femoral arteries than in mesenteric resistance and saphenous arteries. This is likely due to a more pronounced contractile phenotype of smooth muscle cells in small resistance than in large elastic arteries $^{135}$.

All arteries contracted in response to $\alpha_{1}$-adrenergic stimulation, but as in the rabbit ${ }^{129}$, there were regional differences in responses to phenylephrine between vascular beds. However, after intrauterine stress " sensitivity to phenylephrine was decreased and maximal contractile responses to phenylephrine were increased in the renal artery, while responses of mesenteric resistance, femoral and saphenous arteries were unaffected by intrauterine stress. These changes are probably not due to an increased number of $\alpha_{1}$-adrenoceptors, as the density and affinity of $\alpha_{1}$-adrenoceptors in the kidneys were not affected after intrauterine stress. This suggests that the increased responsiveness to $\alpha_{1}$-adrenoceptor stimulation is probably due to alterations in the signal transduction pathway that is engaged.

In the rat renal vasculature $\alpha_{1}$-adrenoceptors mediate the action of sympathetic stimulation ${ }^{1136}$ and an increased renal vascular $\alpha_{\uparrow}$-adrenergic responsiveness is associated with hypertension in adult spontaneously hypertensive rats $^{137}$. Therefore the increased renal arterial response to stimulation with phenylephrine in rats that suffered intrauterine stress, might lead to the development of hypertension later in life. It is also possible that the increased renal $a_{1}$-adrenergic responsiveness causes an imbalance between afferent and efferent arteriolar tone ${ }^{138}$, which can lead to an altered glomerular filtration and renall damage on the long run.

Hyperresponsiveness of blood vessels from hypertensive subjects to contractile agonists has long been known. One of them, 5hydroxytryptamine, has numerous vascular effects, mediated by at least $5 \mathrm{HT}_{2}$-receptors and possibly $\mathrm{a}_{1}$-adrenoceptors ${ }^{139.140}$ reported that the reactivity of the renal vasculature to 5-hydroxytryptamine is enhanced in spontaneously hypertensive rats. In our experiments, stimulation of the renal 
arteries with 5-hydroxytryptamine showed an increased contractile response after intrauterine stress, without altered sensitivity to the agonist.

Maximal contractile responses to exogenously applied norepinephrine were significantly reduced in renal arteries after intrauterine stress, even though intrauterine stress increased $a_{1}$-adrenoceptor mediated responses. Mesenteric resistance, femoral and saphenous arteries were not changed after intrauterine stress and as demonstrated before in 20 day old normal rats ${ }^{141}$, femoral arteries showed only weak responses to norepinephrine. Ozaki et al. studied the effect of dietary restriction in pregnant rats on vascular function in the offspring at different ages and found blunted contractile responses to phenylephrine and norepinephrine in the femoral artery of 20-day-old rats after moderate maternal undernutrition ${ }^{141}$. In contrast to this study and experiments of our own group in the chicken embryo after chronic hypoxia ${ }^{3}$, we did not observe any changes of responses to norepinephrine in the femoral artery. The model of placental insufficiency used in the present study involves reduced fetal supply of both oxygen and nutrients. The combination of both stimuli and the acute nature of the insult might have different effects on vascular function than the separate interventions.

The reduced renal arterial response to norepinephrine, despite an increased renal contractile response to $\alpha_{1}$-adrenoceptor stimulation, might be explained by an enhanced renal dilator response to $\beta$-adrenoceptor stimulation. After intrauterine stress, maximal renal arterial relaxing responses to isoproterenol were increased, while responses of mesenteric resistance, femoral and saphenous arteries to isoproterenol were not modified. The same results were observed after intrauterine stress induced at day 17 of pregnancy. Thus, intrauterine growth acceleration (after intrauterine stress on day 13) as well as intrauterine growth restriction (after intrauterine stress on day 17) is accompanied by the same persistent effects on renal arterial $\beta$-adrenergic reactivity. The increased $\beta$-adrenergic response seems to be in contrast with findings demonstrating decreased $\beta$-adrenergic vasodilatation in hypertension and in ageing ${ }^{142}$. The $\beta$-adrenoceptor requires GTP-binding proteins (G-proteins) to link receptor activation to adenylyl cyclase. Activated $\beta$-adrenoceptors can stimulate both $G_{s}$ (stimulatory G-protein), and the inhibitory $G$-protein, $G_{i}{ }^{19}$. After a period of intrauterine stress, released catecholamines might activate $\beta$-adrenoceptor mediated responses and redirect the cardiac output to tissues that have an increased oxygen demand. Repeated administration of $\beta$-adrenoceptor agonists decreased neonatal $G_{i}$ expression and enhanced $G_{s}$ function ${ }^{143^{144}}$. Increased release of norepinephrine during intrauterine stress throughout late gestation ${ }^{145}$ may continuously activate $\beta$-adrenoceptors and alter postnatal development of $\beta$ adrenoceptor regulation. Why this leads to increased $\beta$-adrenergic reactivity 
in renal arteries but not in other peripheral arteries and how this can persist for at least 3 to 4 weeks after the initial insult, remains to be explored.

To analyse the selectivity of the enhanced $\beta$-adrenoceptor mediated relaxing responses, we used forskolin to directly activate adenylyl cyclase ${ }^{146}$. All vessels fully relaxed in response to this diterpene and intrauterine stress did not alter maximal responses or sensitivity to this compound. Also for calcitonin gene-related peptide, which acts primarily via the activation of adenylyl cyclase under our experimental conditions ${ }^{42}$, intrauterine stress did not alter arterial responses. The observed selectivity might imply that intrauterine stress modifies the density of $\beta$-adrenoceptors or their coupling to adenylyl cyclase. Future experiments are required to strengthen this hypothesis.

Nerve densities differ markedly between vascular beds. Renal arteries, which were hardly innervated with catecholamine-containing nerves, showed small responses to electrical field stimulation. It has been demonstrated that neonatal sympathetic denervation reduces maximal contractile activity and thickness of the media in the adult ${ }^{97}$. However, intrauterine stress did not change these arterial properties in any of the tested vessels nor did a negative fetal environment change sympathetic nerve densities and contractile responses to sympathetic nerve stimulation with electrical field stimulation. Studies from our own group in the chicken embryo demonstrated an increased sympathetic innervation after chronic moderate hypoxia ${ }^{3}$. Yet, these were found not to persist at 21 days of age ${ }^{147}$. A balance between the increased contractile response to $\alpha_{1}$-adrenoceptor stimulation and the increased relaxing response to $\beta$-adrenoceptor activation might result in the maintenance of normal renal arterial responses to nerve stimulation after intrauterine stress. Alternatively, cotranmitters, such as neuropeptide $Y$ and ATP, may help maintain renal arterial neurogenic vasoconstrictor responses. Several studies in animals ${ }^{74,148}$, as well as in humans ${ }^{149,150}$ " have demonstrated that negative events during pregnancy have major consequences for renal hemodynamics and glomerular density and size, which might be linked to the development of cardiovascular disease. Here, glomerular density and size were not modified in 21-day-old rats. In the rat, nephrogenesis begins around day 12 of gestation and is not complete until eight days after birth ${ }^{47}$. Nephrogenesis involves the rapid remodelling of structures, which requires massive apoptosis ${ }^{151,152}$ and renal apoptosis in the rat peaks late in gestation ${ }^{153}$. Thus, the induction of intrauterine stress, at day 13 of gestation, might be a too early time point to dramatically change normal nephrogenesis. This does not suggest that renal function cannot be affected by the changed arterial adrenergic reactivity. The kidneys play an important role in the regulation of blood pressure and renal nerves already influence renal function during development. A variety of functions, including renin secretion, salt and water balance, gluconeogenesis and 
vasoconstriction can be regulated by renal $\alpha$ - and $\beta$-adrenoceptors ${ }^{154}$. Therefore, a normal development of renal adrenoceptor function seems to be important for normal kidney function in the adult.

Our results demonstrated that a suboptimal fetal environment alters renal vascular adrenergic and serotonergic function, at least until four weeks after the insult, while other areas of the arterial tree are not noticeably affected. Whether these changes are persistent, regressive or progressive throughout adulthood and whether they have major consequences for the development of hypertension or renal dysfunction remains to be established. 


\section{Reduced uteroplacental blood flow alters renal arterial reactivity and glomerular properties in the rat offspring}

Marijke W. Sanders, Gregorio E. Fazzi, Ger M. J. Janssen, Peter W. de Leeuw, Carlos E. Blanco, Jo G. R. De Mey

Hypertension. 2004; 43(6): 1283-1289 


\begin{abstract}
Fetal malnutrition and hypoxia may modify organ system maturation and result in cardiovascular diseases in the adult. We tested whether intrauterine stress (IUS) leads to persistent alterations of renal biology. In rats, intrauterine stress was induced by ligation of the uterine arteries at day 17 of pregnancy. Renal arteries of the 21 days old male offspring were isolated to study pharmacological reactivity. Kidneys were dissected to analyze renal structure and $\beta$-adrenoceptor expression. At 21 days of age, half of the animals underwent unilateral left nephrectomy. At the age of 12 weeks, rats were instrumented for blood pressure monitoring, blood sampling and renal function measurements. After intrauterine stress, litter size and birth weight were reduced, while the hematocrit was increased. Renal arterial responses to $\beta$-adrenergic stimulation and sensitivity to adenylyl cyclase activation were increased, along with the renal expression of $\beta_{2}$-adrenoceptors. At 21 days and 6 months of age the number and density of the glomeruli were reduced, while their size was increased. The filtration fraction and urinary albumin concentration were increased 12 weeks after intrauterine stress. In control rats, removal of the left kidney at 21 days of age did not affect kidney function and blood pressure. However, after intrauterine stress, the remaining right kidney failed to compensate for the loss of the left kidney and blood pressure was increased. In conclusion, prenatal stress transiently modifies renal arterial reactivity and results in long lasting adverse effects on renal structure and function and on renal compensatory mechanisms.
\end{abstract}




\section{Reduced uteroplacental blood flow alters}

\section{renal arterial reactivity and glomerular properties in the rat offspring}

\section{Introduction}

Cardiovascular, endocrine and metabolic diseases can emerge as a consequence of intrauterine stress ${ }^{4}$. A suboptimal fetal environment is often the result of placental insufficiency, which leads to an inadequate delivery of nutrients and oxygen to the fetus. Many animal models have been established that support a link between prenatal conditions and the development of disease in the adult. These include maternal caloric and protein restriction ${ }^{2}$, exposure to hypoxia ${ }^{3}$ and ligation of the uterine arteries to mimic placental insufficiency.

Hypoxia and malnutrition during fetal life were reported to affect the reninangiotensin system, the hypothalamic-pituitary-adrenal $\mathrm{axis}^{7}$, vascular endothelial function and sympathetic innervation ${ }^{3,6,8}$ and the development of organs like the kidney ${ }^{10}$. The number of glomeruli can be permanently reduced by influences during fetal life. These structural changes may contribute to renal dysfunction ${ }^{14}$ and hypertension ${ }^{15}$ later in life. Other aspects of renal organogenesis, such as renal vascular development and in particular adrenoceptor mediated renovascular responses ${ }^{13}$, might also be influenced by disturbed fetal growth. In the rat ${ }_{\text {, an unfavorable intrauterine }}$ environment, induced by uterine artery ligation, modified postnatal renal arterial adrenergic reactivity in a regionally selective manner ${ }^{155}$. This led us 
to the hypothesis that intrauterine stress (IUS) results in long lasting alterations of renovascular function and glomerular structure, which may blunt structural and functional compensatory mechanisms in the kidneys and lead to the development of hypertension later in life.

To test this hypothesis, IUS was induced by bilateral ligation of the uterine arteries at day 17 of pregnancy in rats. At 21 days of age, when nephrogenesis is completed ${ }^{47}$ and the vasculature displays most of its pharmacological properties ${ }^{456}$ " renal structure and arterial reactivity were evaluated. To investigate the long-term consequences of IUS for the adult offspring, the animals were instrumented for measuring glomerular filtration rate, renal blood flow and blood pressure. Some of the experiments in 12 week old animals were performed after unilateral nephrectomy at 21 days of age.

\section{Materials and methods}

Experiments were approved by the local ethical committee for animal research of the University of Maastricht. Wistar rats had free access to pelleted food and tap water and were maintained on a 12-h light/dark cycle at $21^{\circ} \mathrm{C}$.

\section{Induction of intrauterine stress (IUS)}

The induction of intrauterine stress (IUS) was performed as described before ${ }^{155}$. Additionally, for identification purposes a toe was amputated from the offspring at birth and blood was collected with micro hematocrit tubes ( 9 $\mu \mathrm{l}$, Modulohm, Herlev, Denmark) to measure hematocrit values ( $7 \mathrm{~min}$. in hematocrit centrifuge). Immediately after this, litter size of the control group was reduced to match that of the IUS group. Not more than two littermates from one mother were used in each experimental group. Because long lasting consequences of the fetal environment might be influenced by gender ${ }^{15 \%}$, the present study was restricted to male offspring. The timing of experimental procedures is summarized in figure 4.1 .

\section{Arterial reactivity}

Left and right segments of the common renal arteries were isolated from the 21-day-old male offspring of both groups and were studied in myographs as described by Sanders et al. ${ }^{455}$.

Morphometric analysis of the arteries and their adrenergic nerve fibers.

Structural properties of renal arteries were determined according to the morphometric methods described by Stassen et al ${ }^{110}$. Glyoxylic acid was used to visualize and quantify periarterial adrenergic nerve fibers in whole mount preparations ${ }^{3}$. 


\section{Mathers}

Sham procedure $(n=16)$<smiles>[13CH3]</smiles>

CON

(male offspring)

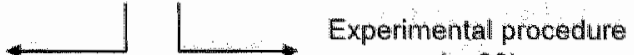

$(n=22)$

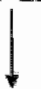

IUS

(male offspring)

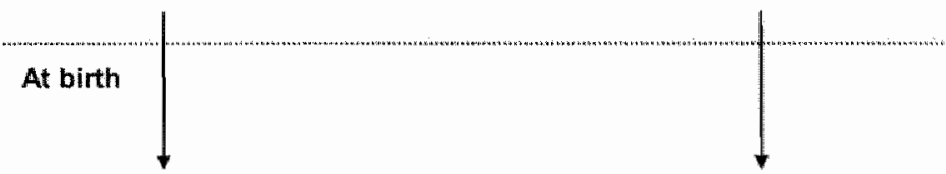

birth weight $(n=204)$, birth weight $(n=137)$,

hematocrit $(n=41)$

hematocrit $(n=77)$

litter size (adjustment)

litter size

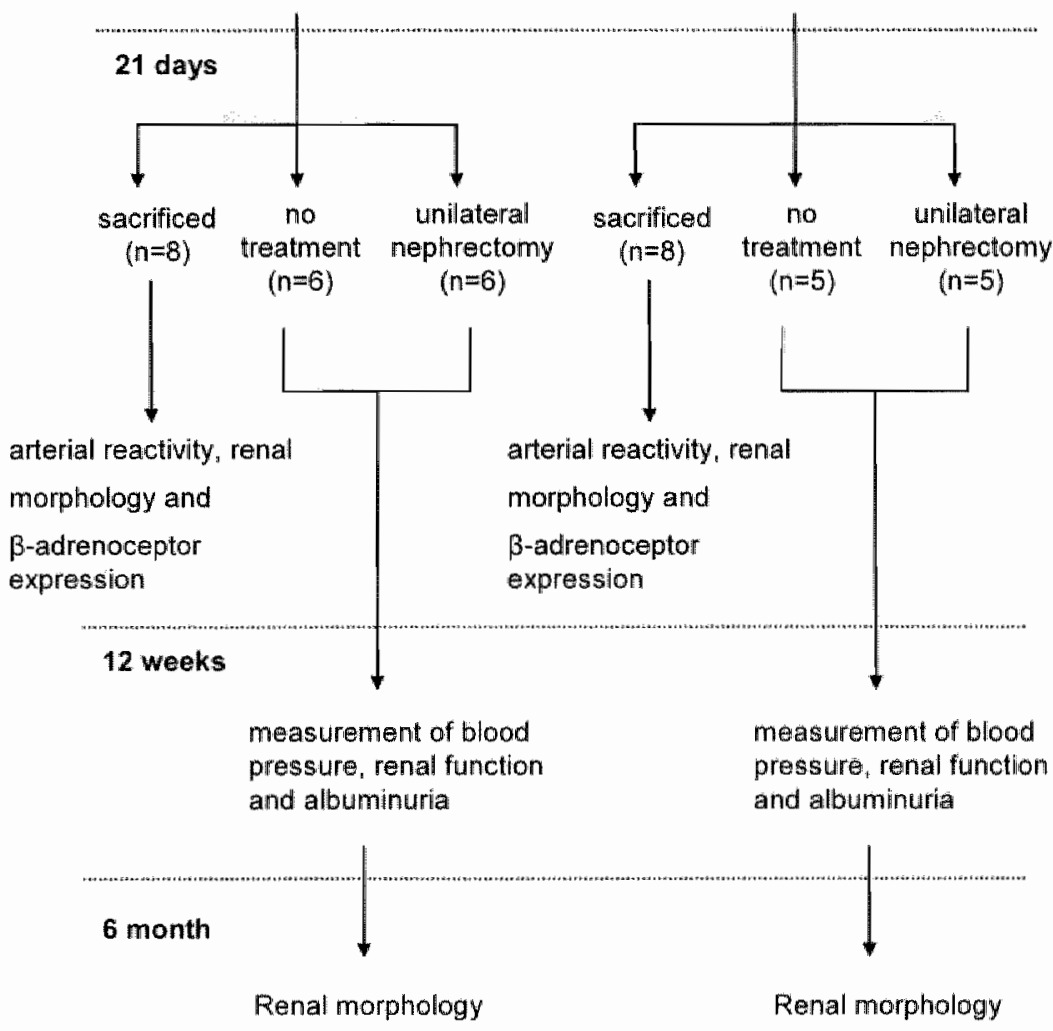

Figure 4.1. Timing of experimental procedures. 


\section{$\beta$-adrenoceptor mRNA expression}

Kidneys were rapidly removed and snap-frozen in liquid nitrogen and stored at $-80^{\circ} \mathrm{C}$ until further processing. The determination of gene expression of $\beta-$ adrenoceptor subtypes was performed as described by Fujinaga and Scott ${ }^{15 \%}$. Alterations in gene expression were determined by changes in the ratio of the intensity of the genes encoding $\beta$-adrenoceptor subtypes and the intensity of the housekeeping gene GAPDH.

\section{Morphometric analysis of the kidneys}

Kidneys of 21 day and 6 months old rats were dissected and embedded in paraffin. Parallel transversal sections were stained with Jones methenamine silver. Glomerular properties were measured and the number of glomeruli within a known volume was calculated according to the formula: $n=G / F \times A$ $x(D+T)^{130.155}$.

\section{Unilateral nephrectomy}

Previous observations have shown that right renal arteries of young rats displayed an increased $\beta$-adrenergic mediated arterial dilatation after surviving uterine stress, compared to control rats ${ }^{155}$. For this reason rats were anesthetized (ketamine, $40 \mathrm{mg} / \mathrm{kg}$ i.p and xylazine $3 \mathrm{mg} / \mathrm{kg} \mathrm{s.c.)} \mathrm{and}$ subjected to left-sided nephrectomy via a paravertebral approach to investigate whether the remaining right kidney could compensate for the loss of the other one.

\section{Instrumentation and renal hemodynamics}

At the age of 12 weeks, rats were anesthetized with ketamine $(40 \mathrm{mg} / \mathrm{kg}$ i.p) and xylazine ( $3 \mathrm{mg} / \mathrm{kg}$ s.c.) and instrumented for blood pressure monitoring and the measurement of inulin (inutest "Laevosan Gesellschaft, Linz, Austria) and para-aminohippurate (PAH, MSD West Point, PA, USA) clearances to evaluate renal hemodynamic function in conscious unrestrained animals. The procedure of instrumentation and the clearance techniques were as described by Fischer et al. ${ }^{159}$.

\section{Plasma creatinine}

Plasma creatinine concentrations were measured using the standard Jaffé technique on a Hitack 747 analyzer (Boeringer Mannheim, Mannheim, Germany).

\section{Albumin assay}

Rats were kept in metabolic cages for 24 hours. Urine was collected and kept at $-20^{\circ} \mathrm{C}$ until further processing. Urine samples were centrifuged $(1500$ $\mathrm{rpm}, 10 \mathrm{~min}$.) and diluted in distilled water. Albumin concentrations were 
measured with the rat albumin enzyme immunoassay obtained from SPI-BIO (Massy Cedex, France).

\section{Data analysis}

Concentration response curves were analyzed in terms of sensitivity $\left(\mathrm{pD}_{2}=\right.$ - $\log E C 50$ ) and maximal response (Emax) by fitting individual concentrationresponse data to a sigmoid regression curve and interpolation (Graphpad Prism version 2.01, Graphpad Software Inc.). Differences between findings in arteries from both groups of rats were tested with Student $t$ test or MannWhitney $U$ test when normality test (Kolmogorov-Smirnov) failed. A value of $p<0.05$ was considered statistically significant. Data are presented as mean \pm S.E.M.

\section{Results}

\section{At birth}

Bilateral ligation of the uterine arteries at day 17 of pregnancy resulted in significantly reduced birth weights $(5.03 \pm 0.05 \mathrm{~g}$ versus $5.35 \pm 0.04 \mathrm{~g}$, $n=137, n=204, p<0.001)$ and litter size $(5 \pm 1$ versus $11 \pm 1, n=22, n=16$, $p<0.001)$. Hematocrit at birth was increased compared to control animals $(0.44 \pm 0.01$ versus $0.39 \pm 0.01, n=77, n=41, p<0.001)$.

Table 4.1. Morphological properties of kidneys of young and adult rats.

\begin{tabular}{|c|c|c|c|c|}
\hline & CON & IUS & CON-UNX & IUS-UNX \\
\hline \multicolumn{5}{|l|}{21 days } \\
\hline Kidney weight $(g)$ & $0.27 \pm 0.01$ & $0.27 \pm 0.03$ & & \\
\hline \multicolumn{5}{|l|}{ Glomerular } \\
\hline Density $\left(/ \mathrm{mm}^{3}\right)$ & $522 \pm 41$ & $360 \pm 29 *$ & & \\
\hline Area $\left(\mu m^{2}\right)$ & $2125 \pm 75$ & $2645 \pm 130^{*}$ & & \\
\hline Circumference $(\mu \mathrm{m})$ & $176 \pm 3$ & $196 \pm 5^{*}$ & & \\
\hline Diameter $(\mu \mathrm{m})$ & $56.1 \pm 1.0$ & $62.3 \pm 1.5^{*}$ & & \\
\hline \multicolumn{5}{|l|}{ Adult (6 months) } \\
\hline Kidney weight $(g)$ & $1.69 \pm 0.09$ & $1.93 \pm 0.1$ & $2.90 \pm 0.12$ & $2.97 \pm 0.17$ \\
\hline \multicolumn{5}{|l|}{ Glomerular } \\
\hline Density $\left(/ \mathrm{mm}^{3}\right)$ & $260 \pm 16$ & $185 \pm 7^{*}$ & $165 \pm 11$ & $129 \pm 7^{* * *}$ \\
\hline Area $\left(\mu \mathrm{m}^{2}\right)$ & $8316 \pm 268$ & $9602 \pm 162^{*}$ & $11066 \pm 502$ & $12779 \pm 433^{* * *}$ \\
\hline Circumference $(\mu \mathrm{m})$ & $343 \pm 6$ & $370 \pm 4$ & $401 \pm 9$ & $429 \pm 8^{* *}$ \\
\hline Diameter $(\mu \mathrm{m})$ & $109 \pm 2$ & $118 \pm 1^{*}$ & $128 \pm 3$ & $137 \pm 3^{ \pm *}$ \\
\hline
\end{tabular}

Control rats (CON), after unilateral nephrectomy at 21 days of age (CON-UNX), rats that survived intrauterine stress (IUS) and after unilateral nephrectomy at 21 days of age (IUSUNX). Values are mean \pm S.E.M.; ${ }^{*} p<0.05$ CON vs. IUS, ${ }^{* *} p<0.05$, CON-UNX vs IUS-UNX. 
A

LETT RENAL ARTERY

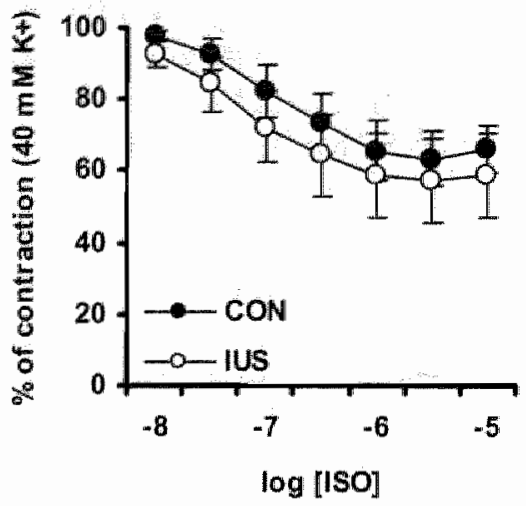

C

LEFT RENAL ARTERY

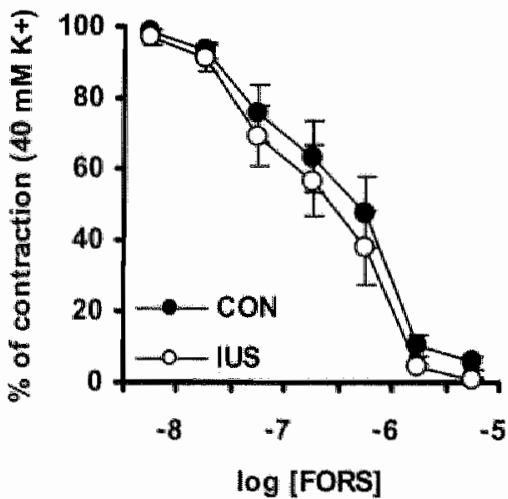

B

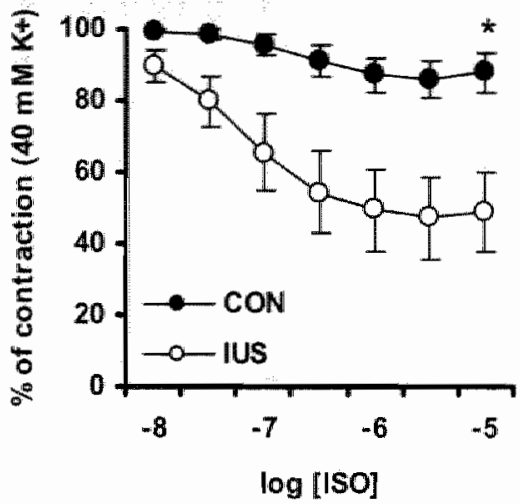

D

RGHT RENAL ARTERY

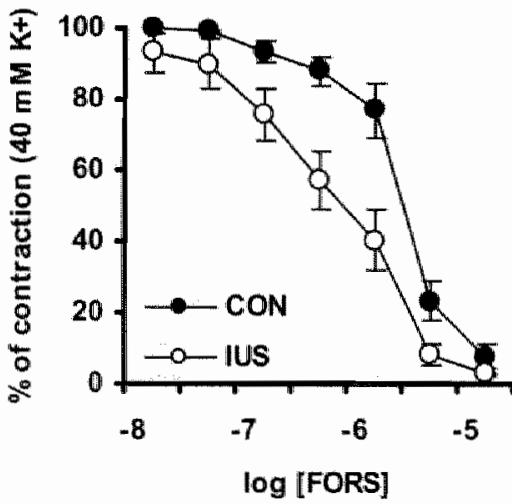

Figure 4.2. Concentration response curves for isoproterenol ( $A$ and $B$ ) and forskolin (C and $D)$ in left and right renal arterles of 21 day old control rats (CON, $)$ and rats that survived intrauterine stress (IUS, O). Data are mean \pm S.E.M., " $p<0.05$ versus control. 


\section{At 21 days of age}

At the age of 21 days, the differences in body weight were no longer present and IUS had not led to variations in organ weights; including kidney weight. However, both kidneys displayed an important decrease in glomerular density $(-31 \%)$, as a result of the prenatal insult. Glomerular area, circumference and diameter were significantly increased (table 4.1), suggesting that IUS induced glomerular hypertrophy. Morphometrical studies of the kidney showed similar results in left and right kidneys. Table 4.1 shows only the results observed in right kidneys for comparison with the remnant right kidney of nephrectomized animals at a later stage of life.

When the animals were 21 days old, renal arteries of the IUS group displayed an asymmetrically altered response to adrenoceptor stimulation. Relaxing responses to $\beta$-adrenoceptor stimulation with isoproterenol in left renal arteries were not affected by IUS (fig. 4.2.a). However, responses and sensitivity of right renal arteries to isoproterenol were significantly increased as a result of IUS (fig. 4.2.b. $\mathrm{pD}_{2}, 7.02 \pm 0.14$ vs $6.62 \pm 0.08, p<0.026$ ). Experiments with forskolin showed that the augmented responses of the right renal artery to isoproterenol were accompanied by an increased sensitivity to direct activation of adenylyl cyclase (fig. 4.2.d. $\mathrm{pD}_{2,} 6.37 \pm 0.19$ vs $5.78 \pm 0.07, p<0.030$ ). This was not observed in the left renal artery (fig. 4.2.c). Compared to control, $\beta_{2}$-adrenoceptor mRNA expression was significantly increased in the right kidneys after IUS (table 4.2). Yet, IUS did not affect the expression of $\beta_{2}$-adrenoceptors in the left kidneys, nor did it change the expression of $\beta_{1}$-adrenoceptors.

Table 4.2. B-adrenoceptor mRNA expression in kidneys of 21 day old rats.

\begin{tabular}{lcc}
\hline & CON & IUS \\
\hline Left kidney & & \\
$\beta_{1}$-adrenoceptor & $0.72 \pm 0.13$ & $0.64 \pm 0.13$ \\
$\beta_{2}$-adrenoceptor & $0.56 \pm 0.11$ & $0.68 \pm 0.13$ \\
& & \\
Right kidney & & \\
$\beta_{1}$-adrenoceptor & $0.51 \pm 0.10$ & $0.58 \pm 0.08$ \\
$\beta_{2}$-adrenoceptor & $0.32 \pm 0.08$ & $0.53 \pm 0.05^{\star}$ \\
\hline
\end{tabular}

Gene expressions are expressed as the ratio of the intensity of the gene encoding $\beta$-adrenoceptors and the intensity of the housekeeping gene GAPDH. Values are mean $:$ SEM; $* 0<0.05$, IUS vs CON. 
Maximal contractile responses to a mixture of $125 \mathrm{mmol} / \mathrm{L} \mathrm{K}+10 \mu \mathrm{mol} / \mathrm{L}$ phenylephrine and $10 \mu$ mollL 5-hydroxytryptamine (Tmax) and the media cross-sectional area (MCSA) were not significantly altered after lUS in left (Tmax, $4.01 \pm 0.44$ vs $2.84 \pm 0.19 \mathrm{~N} / \mathrm{m}_{*}$ n.s; MCSA, $20.3 \pm 1.3$ vs $17.2 \pm 1.2$ $x 103 \mu \mathrm{m} 2$, n.s.) as well as right (Tmax, $3.65 \pm 0.45$ vs $3.05 \pm 0.56 \mathrm{~N} / \mathrm{m}, \mathrm{n} . \mathrm{s}$; $\mathrm{MCSA}_{,} 23.7 \pm 2.0$ vs $19.0 \pm 0.8 \times 103 \mu \mathrm{m} 2$, n.s.) renal artery segments.

IUS did not affect the contractile responses of renal arteries to stimulation of $\alpha_{1}$-adrenoceptors with phenylephrine (fig. 4.3.a) and to sympathetic nerve stimulation (fig. 4.3.b). With the use of glyoxylic acid it could be observed that sympathetic nerve fiber density (1) was larger in left renal arteries than in right renal arteries, but (2) not modified at 21 days of age after IUS (fig. 4.4).

A
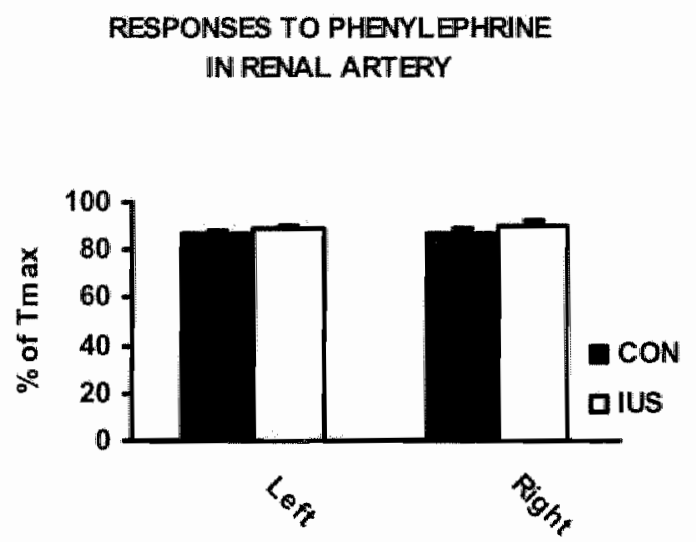

B

\author{
EEECTRICAL FIECD STIMULATION \\ IN RENAL ARTERY
}

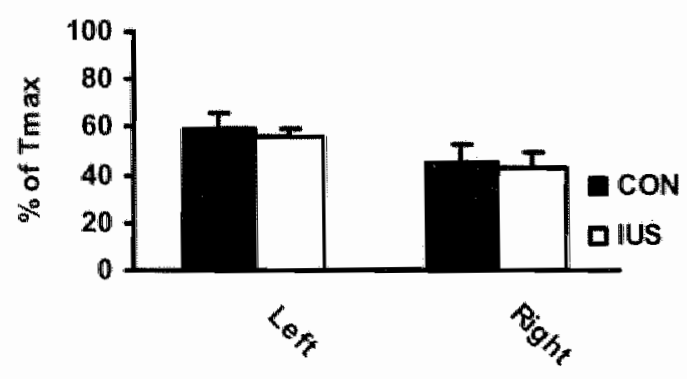

Figure 4.3. Maximal responses to phenylephrine (A) and electrical field stimulation (B) in left and right renal arteries of 21 day old control rats (CON, 1 ) and rats that survived intrauterine stress (US, D). Data are mean \pm S.E.M. 

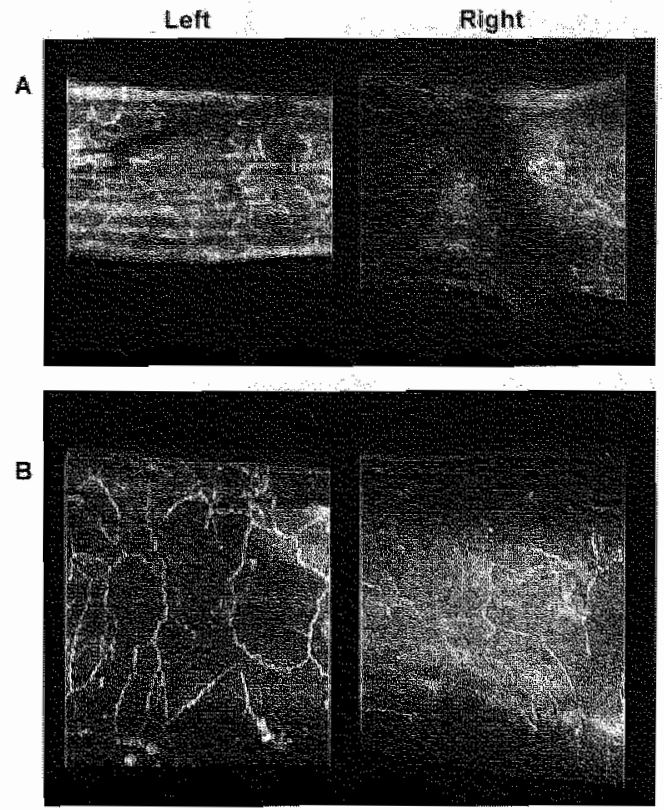

Figure 4.4. Staining of catecholamine containing nerves in left and right renal arteries of 21 day old control rats $(A)$ and rats that survived intrauterine stress $(B)$. Similar results were observed in 6 control and 6 experimental animals.

\section{At 12 weeks of age}

At the age of 12 weeks, all renal arteries failed to relax in response to isoproterenol, regardless of side or intervention (data not shown). However, the IUS induced morphological features of the kidneys had major consequences for renal function at 12 weeks of age. The clearances of PAH (fig. 4.5.a, $+27 \%$ ) and inulin (fig. 4.5.b, $+114 \%$ ) and the filtration fraction (fig. 4.5.c, filtration/perfusion ratio) were significantly increased. However, blood pressure was not affected (fig. 4.5.d). Plasma creatinine concentrations were comparable with control animals, but urinary albumin concentrations were significantly increased, probably as a result of the renal hyperfiltration observed after IUS (table 4.3). 


\section{CON}

$61.2 \pm 6.2$

$985 \pm 145$
IUS

Creatinine $(\mathrm{fmol} / \mathrm{L} / \mathrm{kg})$

Abumin (wg/kg/24h)

After nephrectomy (UNX)

Creatinine (fmol/L/kg)

$75.4 \pm 2.7$

$94.0 \pm 12.8^{*}$

Albumin $(\mu \mathrm{g} / \mathrm{kg} / 24 \mathrm{~h})$
$57.6 \pm 4.0$

$16092 \pm 7004^{*}$

Values are mean $\pm S E M ; ~ * 0<0.05$, IUS vs CON, \# $p<0.05$, IUS ws IUS-UNX.

\section{After unilateral nephrectomy (UNX)}

The renal clearance of PAH and inulin in control animals from which the left kidney had been removed, was not significantly different from that of animals with both kidneys (fig. 4.5). However, when the left kidney was removed from animals that had suffered IUS, the remaining kidney failed to reach the same levels of renal blood flow (PAH) and glomerular filtration rate (inulin) as observed with both kidneys present (fig. 4.5). Blood pressure was not affected by IUS, but unilateral nephrectomy of the left kidney at 21 days of age caused a significant increase in blood pressure at 12 weeks after birth, when compared with IUS alone (fig. 4.5). Plasma creatinine concentrations were significantly increased in nephrectomized animals after IUS, compared to IUS alone, indicating severe renal insufficiency (table 4.3). In IUS animals, nephrectomy did not result in an additional significant increase in urinary albumin concentration. 
A

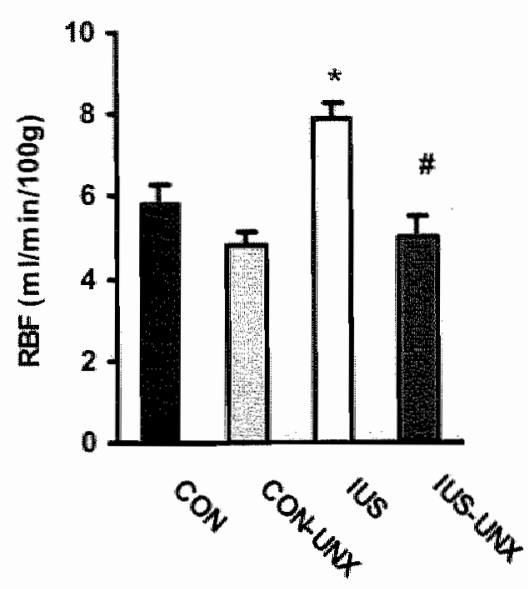

C

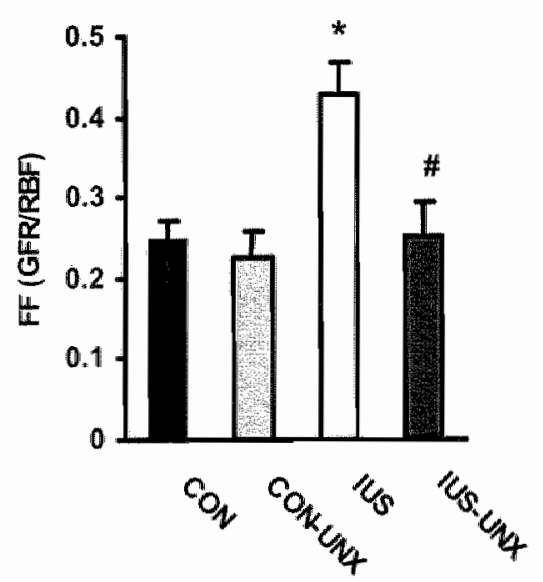

B

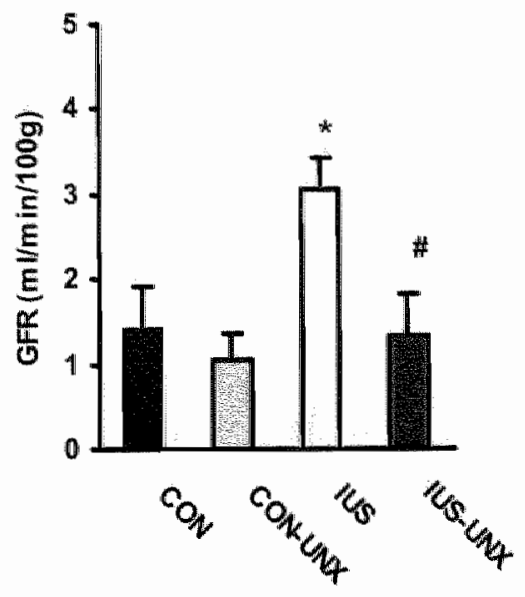

D

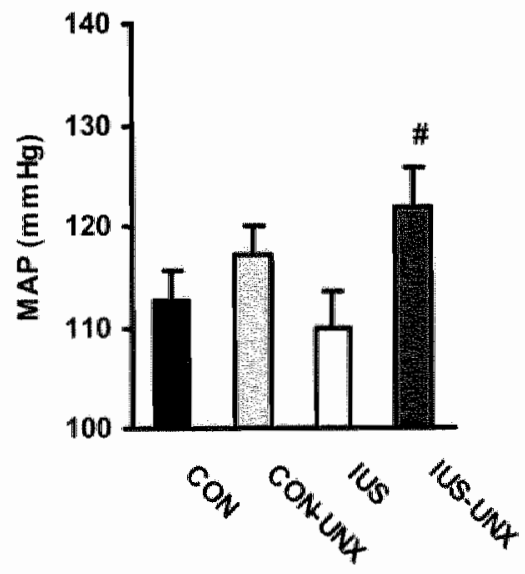

Figure 4.5. $\mathrm{PAH}(\mathrm{A})$ and inuline (B) clearances, filtration fraction (C) and mean arterial pressure (D) in control rats (CON, $\mathbf{m})$, after unilateral nephrectomy at 21 days of age (CON-UNX, in rats that survived intrauterine stress. (UUS, D) and after unilateral nephrectomy at 21 days of age (IUS-UNX, All measurements were performed at 12 weeks of age. Data are mean \pm S.E.M. ${ }^{*} p<0.05$ IUS vs CON, \# $p<0.05$ IUS vs IUS-UNX. 


\section{At 6 months of age}

The reduced density and increased size of the glomeruli, observed at 21 days of age, persisted throughout adulthood (fig. 4.6) and after nephrectomy (table 4.1). However, despite the increased renal perfusion and filtration, we did not observe any histological signs of glomerular disease or glomerulosclerosis (histochemical staining with hematoxylin and eosin) 6 months after IUS with or without additional unilateral nephrectomy.

A

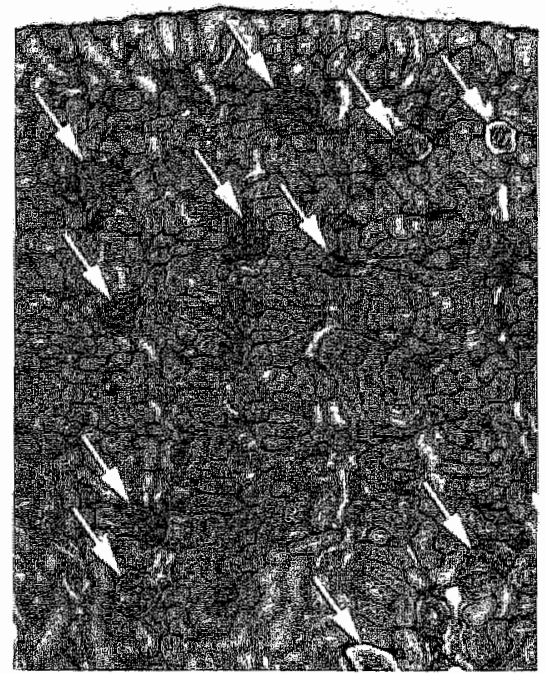

B

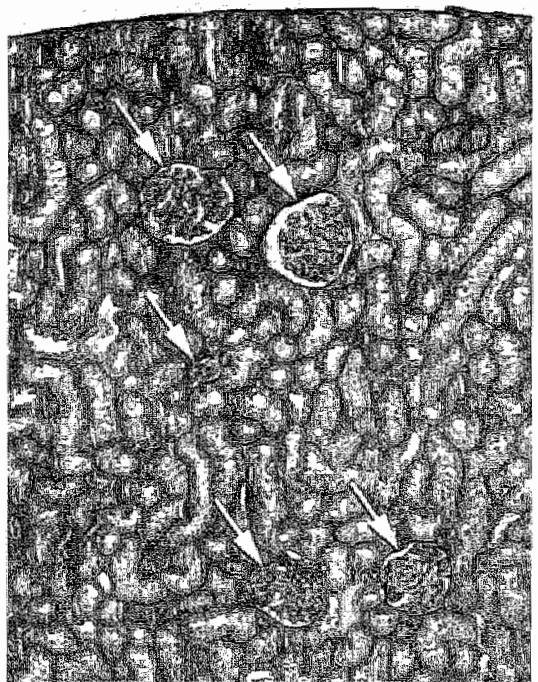

Figure 4.6. Cross-sections (Jones methenamine silver) of kidneys of 6 months old control rats (A) and rats that surviwed ILS (B).

\section{Discussion}

The present study demonstrates a long lasting alteration of the $\beta$-adrenergic system and a reduced glomerular density in the kidneys after IUS. The structural alterations persist in the adult animal and are accompanied by hyperfiltration and hyperperfusion of the glomeruli and by albuminuria, without changing blood pressure. An additional reduction of renal mass by unilateral nephrectomy increased blood pressure in animals that survived IUS. 
IUS was induced by bilateral ligation of the uterine arteries at day 17 of pregnancy $^{6.134}$. The acutely reduced fetal supply of both oxygen and nutrients resulted in a significant reduction of fetal survival and birth weight. Since birth weight seems to be only crude evidence for disturbed development during fetal life ${ }^{2}$, we also determined hematoctrit values at birth. Hematocrit was increased following IUS, indicating that the pups developed in a hypoxic environment during late fetal life or that the plasma volume was reduced as a result of changes in the water and electrolyte balance.

At the age of 21 days, we demonstrated that IUS resulted in an increased sensitivity and maximal relaxing response to isoproterenol in right renal arteries. The augmented responses of the right renal arteries were accompanied by increased $\beta_{2}$-adrenoceptor $\mathrm{mRNA}$ expressions in right kidneys, implying that an increased number of $\beta_{2}$-adrenoceptors is involved in the enhanced responses to isoproterenol. However, this only partially explains the elevated $\beta$-adrenoceptor mediated reactivity after IUS. Isoproterenol acts through $\beta$-adrenoceptors to stimulate adenylyl cyclase. During a period of intrauterine stress, humoral and neurogenic catecholamines might activate $\beta$-adrenoceptor mediated responses ${ }^{13}$ and redirect the cardiac output to tissues that are more vulnerable to hypoxia ${ }^{145}$. Repeated administration of $\beta$-adrenergic agonists has been shown to decrease neonatal $G_{i}$ expression and to enhance $G_{s}$ function ${ }^{119}$. Direct stimulation of adenylyl cyclase showed an increased sensitivity to forskolin, again solely in right renal arteries. These enhanced responses to forskolin suggest that adaptations along the signal transduction pathway contribute to the increased $\beta$-adrenergic renal arterial responses. It has been shown that repeated treatment with isoproterenol increased the number of adenylyl cyclase molecules during development and caused a shift from a $\mathrm{Mn2+}$ stimulated adenylyl cyclase activity to a forskolin-stimulated adenylyl cyclase activity $^{144}$. These findings suggest that an increased release of norepinephrine during IUS throughout late gestation, as was reported for sheep ${ }^{145}$, might continuously activate $\beta$-adrenoceptors and alter postnatal development of $\beta$-adrenoceptor regulation. However, these IUS induced changes of $\beta$-adrenoceptor mediated reactivity in the renal artery did not persist throughout adulthood. Why a negative fetal environment only affects the $\beta$-adrenoceptor mediated responsiveness of the right renal artery is not clear. Yet, it might have important consequence for the development of cardiovascular disease, since van Onna et al. reported an asymmetry of renal blood flow in patients with moderate to severe hypertension ${ }^{160}$. Sympathetic hyperinnervation and vascular smooth muscle hyperplasia may be involved in the development of hypertension in experimental animals ${ }^{11,71}$ and possibly humans ${ }^{44}$. Previous studies from our own group in the chicken embryo demonstrated that chronic moderate hypoxia results in a 
sympathetic hyperinnervation of the arterial system ${ }^{3}$. Additionally, in the rat renal vasculature $a_{1}$-adrenoceptors mediate the action of sympathetic stimulation $^{\text {t3o }}$ and an increased renal vascular $a_{1^{-}}$adrenergic responsiveness is associated with hypertension in adult spontaneously hypertensive rats ${ }^{137}$. Therefore we evaluated the effects of IUS for the $a_{1}$ adrenoceptor and sympathetic nerve mediated responses in renal arteries. Our results presented no lUS-induced differences in response to phenylephrine. Although the rats in this study developed in a hypoxic fetal environment as indicated by the high hematocrit percentages, fluorescent histochemical staining showed that renal arteries were hardly innervated with catecholamine-containing nerves and their densities were not persistently altered as a result of IUS. Maximal contractile responses to nerve stimulation did not significantly differ between renal arteries of rats that survived IUS and control. Although chronic moderate hypoxia results in sympathetic hyperinnervation in the chicken embryo, the model of placental insufficiency used in our experiments involves an acute reduced fetal supply of both oxygen and nutrients. The combination of both stimuli might have different consequences for vascular development than the separate interventions. Moreover, the innervation of rat arteries develops, unlike in the chicken embryo ${ }^{13}$, during the second and third postnatal weeks ${ }^{1 / 1}$. The intrauterine intervention occurred before the appearance of the sympathetic innervation and might therefore not affect nerve densities of renal arteries. Alternatively, after 21 days of postnatal life, arterial innervation might have recovered from IUS induced changes of catecholamine-containing nerves.

IUS did not lead to differences in body weight or organ weights at the age of 21 days. To reach the same body weight as control animals after 21 days, the IUS animals, which had a reduced birth weight, must have had an accelerated weight gain during this early postnatal period. Unlike in humans, nephrogenesis in rat kidneys continues during the first two weeks of postnatal life. Though little is known about the way catch-up growth modifies the effects of low birth weight, it is possible that catch-up growth during this critical period of renal development might have additional consequences for adult renal function and blood pressure.

Morphometric analysis of the kidneys displayed a significant decrease in glomerular density as a result of IUS, while glomerular area, circumference and diameter were significantly increased. These findings suggest that an IUS-induced decrease in glomerular density is a stimulus for the occurrence of glomerular hypertrophy. The reduced glomerular density and the accelerated postnatal growth may result in an excessive demand on the limited number of nephrons. Previous animal ${ }^{74}$ and human ${ }^{150}$ studies have reported a lower than desirable number of functional nephrons as a result of disturbances during fetal growth and described a compensatory increase in glomerular diameter in rats subjected to intrauterine food restriction ${ }^{130}$. It was 
also postulated that fewer nephrons at birth results in the development of hypertension in the adult ${ }^{15}$ and a study in patients with less than $50 \%$ loss of renal mass reported that a higher mean planar glomerular area increases the risk for developing hypertension when there is only mild loss of renal mass. Even though we found a reduced glomerular density $(-31 \%)$ and glomerular hypertrophy, these morphological features did not lead to hypertension in the aduit rat. A more thorough analysis of blood pressure regulation at a later time point, by for instance continuous 24 hour recording of blood pressure, heart rate and their variabilities, may be required to fully appreciate the functional consequences of the structural alterations. However, a strong reduction of renal mass, induced by unilateral nephrectomy at 21 days of age, resulted in a significantly increased blood pressure. A moderate deficiency in glomeruli alone did not result in hypertension, but several lines of observations suggest that individuals with a relatively deficient nephron endowment at birth are predisposed to developing hypertension when subjected to dietary or other stresses ${ }^{54}$. Yet, systemic hypertension is not required for glomerular capillary hyperfiltration and hypertension ${ }^{14}$. Indeed, IUS did not affect blood pressure in our experiments, but we did find a significant increase in glomerular filtration rate, renal blood flow and filtration fraction 12 weeks after IUS, while plasma creatinine values were normal. These findings suggest that IUS resulted in glomerular hypertrophy and hyperfiltration to compensate for the decreased glomerular density and to achieve an adequate renal function, as indicated by the normal plasma creatinine concentrations. Urinary albumin concentrations were significantly increased as a result of IUS. The albuminuria is most likely the result of the increased intraglomerular pressure associated with hyperfiltration and predicts an increased risk for cardiovascular disease and renal functional abnormalities.

A further reduction in the number of glomeruli by unilateral nephrectomy at 21 days of age resulted in an increased blood pressure after IUS and renal insufficiency, as indicated by the high plasma creatinine values and the severe albuminuria. Progressive albuminuria and glomerulosclerosis eventually occur in most experimental models of renal disease characterized by glomerular hyperfiltration and hypertension. However, we did not observe any histological signs of glomerular disease in rats that suffered IUS or additional unilateral nephrectomy. The time point of 6 months might be too early to observe visible signs of glomerular deficiencies as a result of kidney dysfunction. 


\section{Perspectives}

Alterations of kidney development by IUS and possibly catch-up growth induced glomerular hemodynamic changes that resulted in glomerular hyperfiltration, an adaptation seen in response to a reduction in functional nephron number ${ }^{14}$, to achieve an adequate renal function. Hyperfiltration alone was not sufficient to induce an increased blood pressure, but life style factors, such as high salt intake or obesity, coupled with a low nephron number at birth might make animals, which suffered IUS, more susceptible to develop hyperterision.

\section{Acknowledgments}

We thank Dr. Paul Schiffers and Jet Bost for their contributions to this study by their plasma PAH/inuline measurements. 


\section{High sodium intake increases blood pressure and alters renal function in intrauterine growth retarded rats}

Marijke W. Sanders, Gregorio E. Fazzi, Ger M. J. Janssen, Carlos E. Blanco, Jo G. R. De Mey

Hypertension. 2004; 46(1): 71-75 


\section{Abstract}

A suboptimal fetal environment increases the risk to develop cardiovascular disease in the adult. We previously reported that intrauterine stress in response to reduced uteroplacental blood flow in the pregnant rat limits fetal growth and compromises renal development, leading to an altered renal function in the adult offspring. Here we tested the hypothesis that high dietary sodium intake in rats with impaired renal development due to intrauterine stress, results in increased blood pressure, altered renal function and organ damage.

In rats, intrauterine stress was induced by bilateral ligation of the uterine arteries at day 17 of pregnancy. At the age of 12 weeks, the offspring was given high sodium drinking water $(2 \% \mathrm{NaCl})$. At the age of 16 weeks, rats were instrumented for monitoring of blood pressure and renal function. After intrauterine stress, litter size and birth weight were reduced, while hematocrit at birth was increased. Renal blood flow, glomerular filtration rate and the glomerular filtration fraction were significantly increased after intrauterine stress. High sodium intake did not change renal function and blood pressure in control animals. However, during high sodium intake in intrauterine stress offspring, renal blood flow, glomerular filtration rate and the filtration fraction were decreased and blood pressure was increased. In addition, these animals developed severe albuminuria, an important sign of renal dysfunction.

Thus, a suboptimal fetal micro-environment, which impairs renal development, results in sodium-dependent hypertension and albuminuria. 


\section{High sodium intake increases blood pressure and alters renal function in intrauterine growth retarded rats}

\section{Introduction}

A healthy intrauterine environment is prerequisite for normal development. A suboptimal intrauterine environment may permanently alter some tissues and organs that enable the fetus to survive in utero, but cause a predisposition to (cardiovascular) disease later in life". Particularly the development of the kidney can be affected by this process of fetal programming $^{75}$. A possible mechanism might be an impaired nephrogenesis as a result of a suboptimal intrauterine environment. This may lead to reduced nephron endowment during development, which is associated with high blood pressure ${ }^{15}$ and renal dysfunction ${ }^{10}$ in the adult. Previous studies in our laboratory have shown that a suboptimal intrauterine environment, induced by bilateral ligation of the uterine arteries of pregnant rats, resulted in a decreased glomerular number, an increased glomerular size and an altered renal function in the adult offspring. In young adults, we could not demonstrate a change in blood pressure as a result of a suboptimal fetal environment, but we have shown that the renal compensatory capacity after unilateral nephrectomy is reduced ${ }^{162}$.

Not only fetal programming, but also dietary influences such as high sodium intake increase the risk of cardiovascular and renal diseases, independently of other cardiovascular risk factors, including blood pressure ${ }^{163}$. High sodium intake may have detrimental effects on glomerular hemodynamics by inducing glomerular hyperfiltration and increasing the filtration fraction and 
possibly intraglomerular pressure ${ }^{164}$. Furthermore, high dietary sodium can induce left ventricular hypertrophy and is associated with albuminuria ${ }^{163,165,166}$. This led us to the hypothesis that impaired renal development by intrauterine stress (IUS), results in an increased blood pressure, altered renal function and possibly organ damage during high dietary sodium intake.

To investigate this hypothesis, IUS was induced by bilateral distal ligation of the uterine arteries at day 17 of pregnancy in Wistar rats. At the age of 12 weeks, the offspring was given high sodium drinking water $(2 \% \mathrm{NaCl})$ for 4 weeks. At the age of 16 weeks, rats were instrumented for measuring glomerular filtration rate, renal blood flow and blood pressure. After experimentation the animals were sacrificed to determine cardiac and renal structural properties.

\section{Materials and methods}

Experiments were approved by the local ethical committee for animal research of the University of Maastricht. Male and female Wistar rats (Charles River, Maastricht, The Netherlands) had free access to pelleted food and tap water and were maintained on a $12-\mathrm{h} \mathrm{light/dark}$ cycle at $21^{\circ} \mathrm{C}$.

\section{Induction of IUS}

Female rats were anesthetized with ketamine (40 mg/kg i.p) and xylazine ( 3 $\mathrm{mg} / \mathrm{kg} \mathrm{s.c)}$ ). Intrauterine stress (IUS) was induced at day 17 of pregnancy as described before ${ }^{155}$. The offspring of mothers, which underwent bilateral distal ligation of the uterine arteries during pregnancy, are referred to as IUS (intrauterine stress) group and the offspring of mothers ${ }_{i}$ which underwent a sham operation, are referred to as control group. Rats dellivered spontaneously at 23 days and litter size and birth weight of the offspring were determined within one hour after birth. For identification purposes a toe was amputated from the offspring at birth and blood was collected with micro hematocrit tubes ( $9 \mu \mathrm{l}$, Modulohm, Herlev, Denmark) to measure hematocrit values $(7 \mathrm{~min}$. in hematocrit centrifuge). Immediately after this, litter size of the control group was reduced to match that of the IUS group. Because long-lasting consequences of a suboptimal fetal environment can be influenced by gender ${ }^{157}$, the present study was restricted to male offspring.

\section{High sodium diet}

At the age of 12 weeks both groups of animals were given drinking water containing $2 \% \mathrm{NaCl}$ for 4 weeks. During this period, the water intake was measured daily. 
Before and after 4 weeks of treatment with high sodium, the animals were kept in metabolic cages for 24 hours to determine their urinary output.

\section{Instrumentation and renal hemodynamics}

At the age of 16 weeks, rats were anesthetized with ketamine ( $40 \mathrm{mg} / \mathrm{kg} \mathrm{i.p}$ ) and xylazine $(3 \mathrm{mg} / \mathrm{kg}$ s.c). The right femoral artery and vein were cannulated for blood pressure monitoring and the measurement of inulin (inutest; Laevosan Gesellschaft, Linz, Austria) and para-aminohippurate (PAH; MSD, West Point, PA, USA) clearances to evaluate renal hemodynamic function. The procedure of instrumentation and the clearance techniques were as described by Fischer et al. ${ }^{459}$. Measurements were performed 2 days after instrumentation in conscious unrestrained animals.

\section{Morphometric analysis of the left ventricles}

Hearts of 16 weeks old rats were dissected and fixed overnight in phosphate-buffered $(\mathrm{pH} 7.4)$ formaldehyde $(4 \%)$. The tissues were transferred to $70 \%$ ethanol until further processing and eventually embedded in paraffin. Parallel transversal sections were stained with hematoxylin and eosin. Cross-sectional area of the left ventricular wall was measured by subtracting the lumen area from the area enclosed by the outer border of the left ventricle. Left ventricular wall thickness was calculated by dividing the cross-sectional area of the left ventricle by the medial axis of the crosssectional area, using video images generated by a Zeiss Axioscope (Zeiss, Germany), a Leica DFC 280 camera (Leica, Wetzlar, Germany) and commercially available software (Leica Qwin Pro, Wetzlar, Germany).

\section{Renal histology}

Kidneys of 16 weeks old rats were isolated, fixed overnight in phosphatebuffered $(\mathrm{pH} 7.4)$ formaldehyde (4\%) and embedded in paraffin. Parallel transversal sections were stained using Jones methenamine silver of periodic acid Schiff's solution (PAS), to determine whether there was any development of sclerosis or other signs of renal damage.

\section{Albumin assay}

Before the experiment and at the end of the treatment with high sodium drinking water, rats were kept in metabolic cages for 24 hours. Urine was collected and kept at $-20^{\circ} \mathrm{C}$ until further processing. Urine samples were centrifuged (1500 rpm, $10 \mathrm{~min}$.) and diluted in distilled water. Albumin concentrations were measured with the rat albumin enzyme immunoassay obtained from SPI-BIO (Massy Cedex, France). 


\section{Data analysis}

Differences between findings in both groups of rats were tested with a one way ANOVA followed by Bonferroni post hoc test. A value of $p<0.05$ was considered statistically significant. Data are presented as mean \pm S.E.M.

\section{Results}

\section{At birth}

Bilateral distal ligation of the uterine arteries at day 17 of pregnancy resulted in significantly reduced birth weights $(5.03 \pm 0.05 \mathrm{~g}$ versus $5.35 \pm 0.04 \mathrm{~g}$, $n=137, n=204, p<0.001)$ and litter size ( $5 \pm 1$ versus $11 \pm 1, n=22, n=16$, $p<0.001)$ in the offspring. Hematocrit at birth was increased compared to control animals $(0.44 \pm 0.01$ versus $0.39 \pm 0.01, n=77, n=41, p<0.001)$.

\section{Adult}

Before the treatment with the high sodium diet, the body weights of the control and IUS animals were not significantly different. After four weeks of treatment, both groups showed a similar increase in body weight $(+15 \%)$ and there were no significant differences in the sodium induced increases in water intake and urine volume between the two groups (table 5.1).

Table 5.1. Effects of intrauterine stress and high sodium intake on body weight, water intake and urine volume.

\begin{tabular}{lcccc}
\hline & \multicolumn{2}{c}{ Without treatment } & \multicolumn{2}{c}{ With treatment } \\
& CON & IUS & CON-HS & IUS-HS \\
\hline Body weight $(\mathrm{g})$ & $315 \pm 14$ & $339 \pm 8$ & $361 \pm 12^{4}$ & $390 \pm 7^{\ddagger}$ \\
& $(m=6)$ & $(n=5)$ & $(n=8)$ & $(n=10)$ \\
Water intake $(\mathrm{g} / 24 \mathrm{~h})$ & $24 \pm 1$ & $29 \pm 2$ & $52 \pm 6^{\dagger}$ & $65 \pm 9^{\ddagger}$ \\
Urine volume $(\mathrm{m} / 24 \mathrm{~h})$ & $13 \pm 1$ & $13 \pm 1$ & $45 \pm 8^{\ddagger}$ & $46 \pm 12^{\ddagger}$ \\
\hline
\end{tabular}

Control rats (CON) and rats that survived intrauterine stress. (IUS) before and after 4 weeks of treatment with $2 \% \mathrm{NaCl}$ in drinking water (CON-HS and IUS-HS). Values are mean 1 S.E.M." $\dagger p<0.05$ CON vs CON-HS, $¥ p<0.05$ IUS vs IUS-HS.

Renal function and hemodynamics in IUS versus control animals are presented in figure 5.1. The clearances of inulin (fig. 5.1.a) and PAH (fig. 5.1.b) and the filtration fraction (fig. 5.1.c, filtration/perfusion ratio) were significantly increased in IUS animals on normal drinking water. However, blood pressure was not affected (fig. 5.1.d). Figure 5.1a shows that animals that survived IUS did not reach the same level of glomerular filtration rate during high sadium intake than on normal drinking water, while in control 
animals the glomerular filtration rate was maintained during high sodium intake. High sodium intake also reduced renal blood flow (fig. 5.1.b) and filtration fraction (fig. 5.1.c) in IUS animals, whereas control animals did not show sodium-induced changes in these parameters. The decrease in glomerular filtration rate and renal blood flow in IUS animals during a high sodium intake was accompanied by an increased blood pressure (fig. 5.1.d).

A

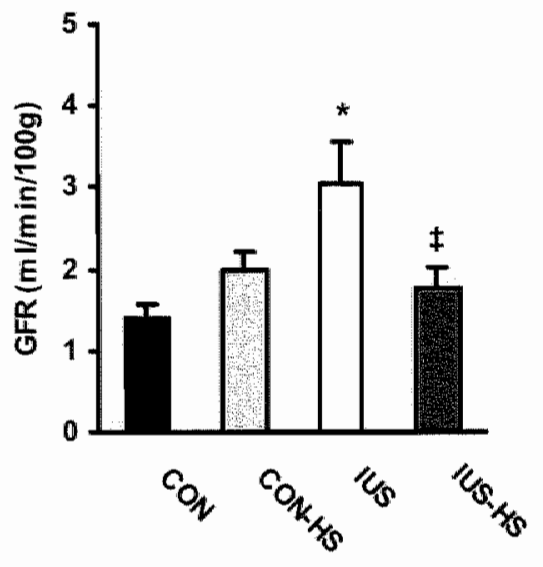

C

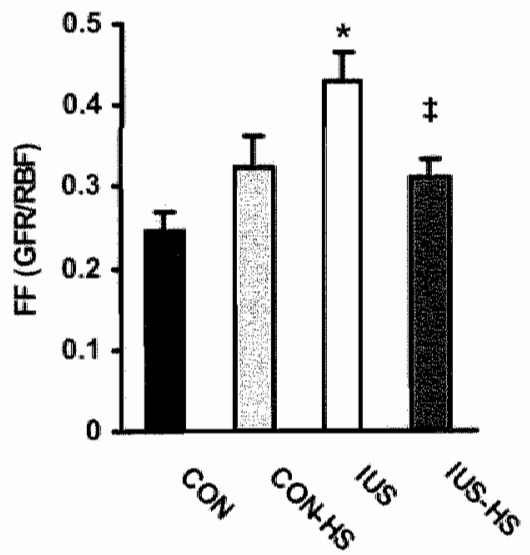

B

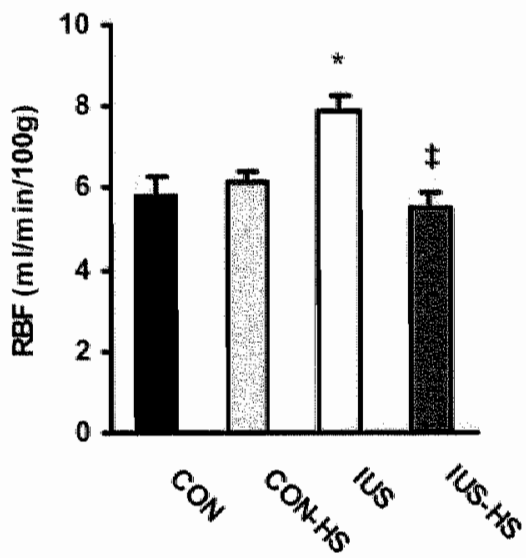

D

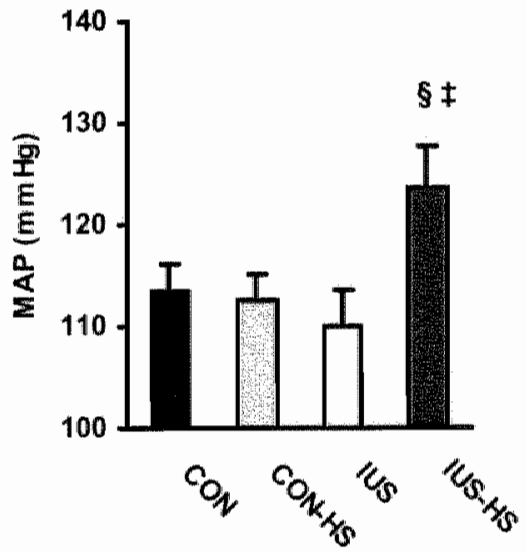

Figure 5.1. Inulin (A) and PAH (B) clearances, fittration fraction (C) and mean arterial pressure (D) in controll rats without (CON, and with 4 weeks of high sodium intake (CON-HS, and in rats that survived intrauterine stress without (IUS, D) and with 4 weeks of high sodiulin intake (IUS-HS, 整). Data are mean \pm S.E.M. ${ }^{*} p<0.05$ IUS vs CON, $\neq p<0.05$ IUS vs IUS-HS, $\$ p<0.05$ CON-HS vs IUS-HS. 
In addition, high sodium treatment led to a significantly increased excretion of albumin in the urine of IUS animals, compared to control animals and compared to animals that survived IUS on normal drinking water (fig. 5.2).

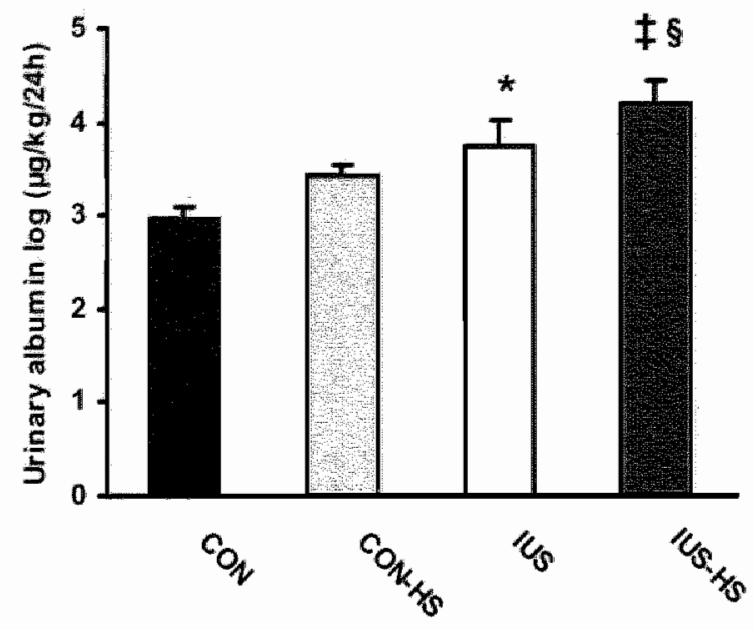

Figuur 5.2. Urinary' albumin excretion in control rats without (CON, $\mathbf{0}$ ), and with 4 weeks of high sodium intake (CON-HS,, ) and in rats that survived intrauterine stress without (US, $\square$ ) and with 4 weeks of high sodium intake (IUS-HS, Data are mean \pm S.E.M. " $p<0.05$ IUS vs CON, $\ddagger p<0.05$ IUS ws IUS-HS, $\S p<0.05$, CON-HS vs IUS-HS.

After sacrificing the animals we measured left ventricular wall thickness to investigate whether animals that survived IUS are more susceptible to develop left ventricular hypertrophy during high sodium intake. However, we could not demonstrate any differences in left ventricular wall thickness between the experimental groups during high sodium treatment (CON-HS vs IUS-HS; $2359 \pm 53$ vs $2467 \pm 43 \mu \mathrm{m}$ ).

Although we previously demonstrated a decreased glomerular number and an increased size of the glomeruli after IUS ${ }^{162}$, we did not observe any additional histological signs of glomerular disease or glomerulosclerosis 4 weeks after treatment with high sodium (data not shown). 


\section{Discussion}

Intrauterine stress (IUS) as a consequence of reduced uteroplacental blood flow limits fetall growth and compromises renal development, which results in an altered renal function in the adult offspring ${ }^{162,167}$. The present study demonstrates that in animals that survived IUS, high sodium intake leads to increased blood pressure and albuminuria.

Though IUS resulted in reduced birth weight, body weights were not significantly different between control and IUS animals at 12 weeks of age. The intervention thus resulted in fetal growth retardation and postnatal catch-up growth, which have been associated with an increased risk for cardiovascular disease in the adult human ${ }^{168}$. During the 4 weeks of treatment with high sodium the body weights increased to the same extent in both groups. The weight gain between 12 and 16 weeks was to be expected because of normal growth of the animals. In both groups of animals high sodium intake resulted in comparable increases in water intake and urinary output.

IUS alone resulted in reduced nephron endowment during renal maturation, followed by an increased glomerular filtration rate, renal blood flow and filtration fraction in the adult ${ }^{162}$. High sodium intake resulted in a significant decrease of these renal hemodynamic parameters. The pathophysiological mechanisms of the altered glomerular hemodynamics in response to sodium loading are not fully elucidated. Glomerular filtration is linked to the extracellular fluid volume by feedback loops that include elements like renal nerves, the renin-angiotensin system and natriuretic peptides or the concentration of sodium in the tubular fluid that reaches the macula densa by tubuloglomerular feedback. These feedback systems compete for the control of glomerular filtration. In the onset of diabetes the kidneys grow and start to hyperfiltrate ${ }^{169}$. It has been proposed that in diabetes tubular hypertrophy increases sodium reabsorption in the proximal tubules and less sodium reaches the macula densa. In the normal rat, the macula densa mediates renin release by sensing decreases in luminal sodium chloride and thereby regulates afferent arteriolar tone ${ }^{170}$. However, reduced salt intake increased renal blood flow in diabetic rats and caused renal vasodilatation in diabetic patients ${ }^{171,172}$. Thomson and Vallon demonstrated that the increase in proximal reabsorption leads to a paradoxical effect of dietary sodium on glomerular filtration rate; a high sodium intake resulted in a decreased glomerular filtration rate and renal vasoconstriction in diabetic rats ${ }^{173.174}$. They found a negative impact of dietary sodium on reabsorption upstream from the macula densa and an important decrease in proximal reabsorption. The increased sensitivity of the proximal tubules in diabetes results in a strong influence of dietary sodium on the tubuloglomerular feedback signal, implying that a higher sodium intake leads to enhanced activation of the 
tubulogllomerular feedback system and vice versa ${ }^{173,175}$. In our study we also observed that the glomerular filtration rate and renal blood flow were increased as a result of IUS and that these parameters were reduced in response to high sodium intake. Possibly, the salt paradox proposed by Thomson et al. ${ }^{173}$ might also be applicable to the altered renal function we observed in animals that survived IUS. IUS alters renal development which might result in tubular hypertrophy and an increased proximal reabsorption and make these tubules more sensitive to dietary sodium intake. More experiments are necessary to confirm this hypothesis. Moreover, high sodium might not only restore glomerular filtration rate to control levels via the tubuloglomerular feedback system, but it might also affect other feedback loops. They may include vasoconstriction of glomerular arterioles due to activation (or inadequate suppression) of neuroendocrine systems ${ }^{163}$, such as the renin-angiotensin system ${ }^{176.177}$, or the sympathetic nervous system $^{178}$. Possibly, these elements play an important role in returning renal blood flow to control levels in IUS animals. Although high sodium intake, via an increased response of renal nerves and the renin-angiotensin system, locally, restores renal hemodynamic function to control levels, systemically, it might be responsible for the increased blood pressure in IUS animals.

An additional manifestation of renal dysfunction is the elevated urinary albumin concentration in IUS animals compared to control animals, before and after the intake of high sodium. High sodium might be harmful to the selective permeability of the glomerular basement membrane and worsen the urinary excretion of albumin in IUS animals. In the glomerulus, filtration takes place across the capillary wall into the Bowman's space. The wall includes three layers: an inner layer of glomerular endothelial cells, an outer (urinary side) layer of glomerular epithelial cells or podocytes and, lying between the two cellular layers, the glomerular basement membrane. It is generally accepted that the glomerular podocyte is the cell primarily responsible for the prevention of albuminuria in health and that podocyte damage/dysfunction underlies albuminuria in disease ${ }^{179}$. Albumin destined for excretion appears obligated to pass through the degradation pathway within lysosomes. The majority of albumin is excreted as low-molecularweight peptide fragments and there is evidence that albuminuria in cardiovascular disease (including diabetic kidney disease, preeclampsia and hypertension) may be the result of factors affecting the degradation pathway of filtered albumin, rather than a primary effect on glomerular permeability. The major underlying factors associated with tissue damage and fibrosis in cardiovascular and kidney disease are the up-regulation and action of growth factors such as transforming growth factor-beta (TGF- $\beta$ ) and cytokines produced in response to changes in systemic factors, particularly blood pressure. TGF- $\beta$ is linked to increased levels of intact albumin in the urine by affecting the albumin uptake and the lysosomal breakdown of 
filtered albumin by proximal tubular cells prior to excretion ${ }^{180,184}$. Additionally" there is an interaction between TGF- $\beta$ and the renin-angiotensin system. Inhibition of the renin-angiotensin system corresponds to suppression of TGF- $\beta$ production, but also activation of lysosomal activity ${ }^{182}$. Perhaps, the increase in blood pressure in IUS animals during high sodium intake raised the activity of TGF- $\beta$ and increased the urinary albumin excretion. Again, more research is needed to provide evidence for this hypothesis.

It has been shown that high dietary sodium leads to the development of target organ damage, like left ventricular hypertrophy and renal fibrosis ${ }^{183,184}$. Yet, in this study, we could not demonstrate any high sodium induced increase of left ventricular wall mass or of renal glomerular or peritubular fibrosis. It is well possible that the duration of the treatment with sodium was not extensive enough to cause visible signs of left ventricular hypertrophy or collagen deposition in the kidneys. On the other hand, studies in sodium sensitive hypertensive patients have described impaired left ventricular diastolic function without any differences in left ventricular wall mass, compared to salt-resistant patients and irrespective of blood pressure ${ }^{185}$. Thus, although these animals did not develop left ventricular hypertrophy, this does not mean that they do not have early signs of left ventricular dysfunction.

\section{Perspectives}

Taken together, our results indicate that IUS and additional high sodium intake results in an altered renal function, albuminuria and an increased blood pressure. This suggests that, although IUS alone does not result in hypertension, it predisposes these animals to be more sensitive to high dietary sodium intake. An unfavorable intrauterine environment might be a factor which interferes with the response to sodium and this could explain why some persons are more susceptible to sodium-induced effects on blood pressure than others.

\section{Acknowledgements}

We thank Dr Paul Schiffers and Jet Bost for their contributions to this study by their plasma $\mathrm{PAH} /$ inulin measurements. 


\section{General discussion}

\section{General}

An insufficient delivery of nutrients and oxygen to the fetus can limit intrauterine growth and enable the fetus to survive in utero, but may permanently alter some tissues and organs. On the long run these alterations can cause a predisposition to (cardiovascular) disease later in life. Intrauterine stress induced at different time points of fetal development and the possible subsequent catch-up growth might have dissimilar effects on the maturation of tissues and organ systems. We therefore investigated the effects of experimental intrauterine stress at an early day 13 of pregnancy) and later (day 17 of pregnancy) stage of gestation in the rat.

To appreciate the consequences of intrauterine stress (IUS) for vascular reactivity we first evaluated the normal development of adrenergic, sympathetic and peptidergic responses in arteries from different vascular beds (chapter 2). Subsequently, we investigated the consequences of a suboptimal fetal setting for these arterial responses in a rat model of placental insufficiency. Chapter 3 and 4 describe that bilateral ligation of the uterine arteries during pregnancy is a suitable model to mimic placental insufficiency and that it has far-reaching consequences for renal arterial adrenergic reactivity. Furthermore, this model of intrauterine stress permanently alters glomerular structure and function in the adult rat and changes the compensatory mechanisms of the kidneys (chapter 4 and 5).

Thus, the studies described in the present thesis provide evidence for an association between adverse intrauterine conditions and alterations in renal vascular reactivity and renal structure and function in the rat 


\subsection{Development of arterial adrenergic and peptidergic reactivity}

Arteries are innervated to a different extend during development and the maturation of adrenergic and peptidergic responses occurs in a distinct way in different vascular beds (chapter 2). During postnatal development, a marked growth of the arterial wall occurs, which seems to be independent of the level of innervation. This suggests that only minor neural input is required to mediate developmental arterial smooth muscle hypertrophy and differentiation. The arterial wall is very sensitive to hemodynamic forces, like increased blood flow and blood pressure and these forces regulate growth and remodeling throughout arterial maturation ${ }^{19,22}$.

Periarterial sympathetic and sensory-motor nerves were already present 6 days after birth. Their densities varied between different parts of the vascular tree and these regional variations were similar for both types of nerves. This could be due to regional differences in the production of nerve growth factors between various parts of the arterial tree ${ }^{27,28}$. Neonatal sympathectomy results in an increased density of CGRP (calcitonin-gene related peptide)containing nerves ${ }^{29}$, while neonatal denervation of sensory-motor nerves has a trophic effect on the development and function of sympathetic nerves ${ }^{30}$. The absence of one type of nerves might increase the availability of nerve growth factor (NGF) for the other type of nerve and this might indicate that the presence of CGRP-containing nerves is a requirement for an efficient development and function of catecholamine-containing nerves. This in turn is necessary for maintaining optimal function of sensory-motor nerves and the development of adrenergic vascular function.

The nerve densities differ between vascular beds and the responses of the tested arteries to nerve stimulation and to the $\alpha_{1}$-adrenergic agonist phenylephrine corresponded with the density of sympathetic nerves. However, in the renal artery the response to nerve stimulation did not match its low density of cathecholamine-containing nerves. This could be explained by the high sensitivity of renal arterial smooth muscle cells to $a_{1}$-adrenergic stimulation. It is also possible that the responses to nerve stimulation and agonists is associated with the electrical coupling between innervated and non-innervated smooth muscle cells, receptor densities and their subsequent second messenger system ${ }^{117}$.

Although the nerve densities of sensory-motor and sympathetic nerves barely changed, the relaxing responses to the $\beta$-adrenergic agonist isoproterenol and endogenous and exogenously applied CGRP progressively decreased during postnatal development, except in the mesenteric resistance artery. Since there was no change in nerve densities during development, the fading of the relaxing responses to CGRP seems to be the result of a reduction of post-junctional effects of the agonists. Both CGRP -and $\beta$-adrenergic receptors are linked to adenylyl cyclase via 
stimulatory Gs proteins, which results in a cAMP-dependent relaxation of the arteries. Direct stimulation of adenylyl cyclase with forskolin demonstrated a developmental decrease in sensitivity, while the maximal response was not altered. Stimulation of the $\beta$-adrenoceptor can activate both $G_{s}$ and the inhibitory $G$ protein, $G_{i}^{119}$. Ageing-related decreases in relaxing responses to $\beta$-adrenergic receptor stimulation have been attributed to increases in $G_{i}^{120}$. Thus, during maturation not only the coupling of the $\beta$-adrenergic and CGRP-receptors to $G_{s}$ proteins is reduced, but also the subsequent signal transduction pathway is progressively blunted.

in summary, during development, several parts of the vascular tree are innervated to a different extend and ingrowing sympathetic and sensory motor nerves may redistribute receptors on the cell surface or can be involved in the post receptor expression of G-proteins or the subsequent signal transduction system. It is important to consider these findings when interventions are planned during fetal development, because a suboptimal intrauterine environment may affect various vascular beds in a different manner.

\subsection{Arterial adrenergic development and intrauterine stress}

In chapter 3 we provide evidence for regionally selective effects of intrauterine stress (IUS) on the development of arterial adrenergic reactivity. IUS induced by bilateral ligation of the uterine arteries at day 13 of pregnancy only altered renal arterial adrenergic function at 3 weeks after birth. Other tested areas of the arterial tree were not modified. Experiments described in chapter 4 highlight these regional differences even further. The regionally selective effects of IUS were not only restricted to renal arteries; they were even far more marked in the right than in the left renal artery. We observed an augmented $\beta$-adrenoceptor mediated response of the right renal arteries which was accompanied by increased $\beta_{2}$-adrenoceptor mRNA expressions in right kidneys, implying that that an increased number of $\beta_{2}$ adrenoceptors is involved in the enhanced response to isoproterenol after IUS. Experiments to investigate the sympathetic innervation and adrenergic function of left and right renal arteries of young (21 days old) and adult (12 weeks) rats showed that the left renal artery was more densely innervated with catecholamine-containing nerves and that the response to electrical field stimulation (data not presented in this thesis) and $\beta$-adrenoceptor stimulation was larger compared to the right renal artery. Why there is this asymmetry in the density and function of the periarterial sympathetic innervation remains to be elucidated. We can only speculate that the left kidney is more specialized in short-term, fast responses, required to adapt to a changed demand of the body. It is not clear why an unfavorable fetal 
environment only affects the $\beta$-adrenoceptor expression and $\beta$ adrenoceptor-mediated responses of the right renal artery. Van Onna et al. reported an asymmetry of renal blood flow in patients with moderate to severe hypertension ${ }^{100}$. So, the asymmetry of the effects of IUS on renal arterial properties might have important consequences for the development of cardiovascular disease.

After IUS induced at day 13 of pregnancy, sensitivity to phenyllephrine was decreased and maximal responses were increased in the renal artery, while responses of the other tested arteries were unaffected. The density and affinity of $a_{1}$-adrenoceptors in the kidneys were not altered by $\| U S$, suggesting that the increased responsiveness to $\alpha_{1}$-adrenoceptor stimulation is probably due to alterations in the signal transduction pathway. An increased renal arterial responsiveness to stimulation of $a_{1}$-adrenoceptors is associated with hypertension in adult spontaneously hypertensive rats ${ }^{137}$ and this implies that the IUS induced hyperresponsiveness to phenylephrine might predispose an individual to develop hypertension later in life. The increased response to $\alpha_{1}$-adrenoceptor activation might also be responsible for the increased contractile reactivity to 5-hydroxytryptamine. 5Hydroxytryptamine stimulates arterial vasoconstriction through 5-HT2 receptors and $\alpha_{1}$-adrenoceptors ${ }^{139}$ and these effects are enhanced in the renal vasculature of spontaneously hypertensive rats ${ }^{140}$.

We also demonstrated an increased response of the renal artery to $\beta$ adrenoceptor stimulation with isoproterenol after IUS. However, the sensitivity and maximal response to direct stimulation of adenylyl cyclase and the arterial responses to CGRP were not modified by IUS, suggesting that IUS selectively alters the density of $\beta$-adrenoceptors or their coupling to adenylyl cyclase. Increased release of norepinephrine during IUS $^{145}$ may continuously activate $\beta$-adrenoceptors and Zeider et al. observed that repeated administration of $\beta$-adrenoceptor agonists decreased neonatal $G_{i}$ expression and enhanced $G_{s}$ function ${ }^{143,144}$. During a period of IUS, released cathecholamines might activate $\beta$-adrenoceptor-mediated responses and redirect the cardiac output to tissues that have an increased oxygen demand. The kidneys are vital organs, which might be vulnerable to hypoxia. It is commonly believed that the fetal cardiovascular hemodynamic response to hypoxia excludes the kidneys. In the fetus, however, normal blood flow to the kidneys is already much lower than in the newborn and in the adult. The results of studies concerning fetal renovascular responses to hypoxia are conflicting. Depending on the severity of hypoxia and the way it is applied, a decrease or an increase of renal blood flow has been reported ${ }^{186}$. Although we do not know the exact nature of the hypoxic insult in our study, a possible explanation for the observed increased $\beta$-adrenergic reactivity in renal arteries and not in other peripheral arteries could be that the increased $\beta$ adrenergic reactivity of the renal artery is a mechanism to increase renal 
blood flow during hypoxemia to protect the vulnerable areas of the fetal kidney to ischemic-hypoxic damage. The mechanisms, through which these regionally selective alterations in arterial reactivity persist for weeks after the initiating insult, remain to be elucidated.

Bilateral ligation of the uterine arteries on day 13 or 17 of pregnancy resulted in the same persistent effects on renal arterial $\beta$-adrenergic reactivity. However, the induction of IUS during late pregnancy resulted in the expected growth retardation, as previously reported in several human and animal studies, while IUS, induced early during pregnancy, even led to prenatal growth acceleration (catch-up growth). To reach the same weight as control animals at 21 days of age, the animals that survived IUS, induced at day 17 of pregnancy, must have had an accelerated weight gain during the first 3 weeks of postnatal life. Little is known about how catch-up growth influences vascular development, but catch-up growth in utero might have different effects than postnatal catch-up growth.

These findings imply that birth weight is not an ideal marker of exposure to a suboptimal fetal environment. Studies performed by our own group recently reached a similar conclusion. Both protein malnutrition and chronic moderate hypoxia in the chicken embryo induced growth retardation "but had distinct effects on arterial endothelium-dependent reactivity ${ }^{2,70,127}$. Since birth weight seems to be only crude evidence for disturbed fetal development, we also determined hematocrit values in newborn rats that survived IUS induced at day 17 of pregnancy. We found that hematocrit was increased after IUS, indicating that the offspring developed in an environment that was more hypoxic than normal. Alternatively, a decreased plasma volume of the newborns due to changes in the water and electrolyte balance may have contributed to the increased hematocrit.

Although both early and late induction of IUS modified renal vascular reactivity, there were some major discrepancies in the consequences of IUS for vascular reactivity between the two time points of intervention. We did not observe any modification of $a_{1}$-drenoceptor-mediated responses after IUS, induced at day 17. Additionally, in contrast to the early intervention, direct stimulation of adenylyl cyclase showed an increased sensitivity to forskolin This suggests that, next to an increased expression of $\beta_{2}$-adrenoceptor mRNA, the signal transduction system is involved in the enhanced response to $\beta$-adrenoceptor activation. The continuous stimulation of $\beta$-adrenoceptors by norepinephrine during IUS throughout late gestation might, beside a shift in the expression of $G$ proteins from the $G_{i}$ to the $G_{s}$ type, cause an increase in the number of adenylyl cyclase molecules during development and a change from a Mn2+-stimulated adenylyl cyclase activity to a forskolinstimulated adenylyl cyclase activity ${ }^{144}$. Thus, $\beta$-adrenoceptor activity is regulated by several different mechanisms and the time-point of the 
intervention during fetal development seems to be important in the regulation of the $\beta$-adrenoceptor-mediated arterial response.

In conclusion, different time-points of intervention and of subsequent catchup growth seem to have different consequences for renal adrenergic arterial development. Renal $\alpha$ - and $\beta$-adrenoceptors regulate a variety of functions, including renin secretion, salt and water balance, gluconeogenesis and vasoconstriction $^{154}$. For that reason, both interventions can have major consequences for kidney function in the adult.

\subsection{Effects of intrauterine stress on glomerular structure and function}

The different effects of the two time-points of intervention were not limited to modifications of renal arterial reactivity. IUS induced at day 17 of gestation resulted in a decreased glomerular density in young rats, while we could not observe any alterations in glomerular number after the induction of IUS at day 13 of gestation. In humans, nephrogenesis starts at midgestation and is completed before term ${ }^{187}$. In the rat, however, nephrogenesis starts around day 12 of gestation and is not complete until postnatal day $8^{47}$. Nephrogenesis involves the rapid remodeling of structures, requiring massive apoptosis ${ }^{151,152}$. In the rat, renal apoptosis peaks late in gestation ${ }^{153}$ and this might explain why the induction of IUS early during pregnancy did not affect glomerular number. Several studies have also indicated that an intact renin-angiotensin system is a prerequisite for normal renal development and especially an intact signaling through the $\mathrm{AT}_{1}$-receptors is important for nephron development ${ }^{188}$. Woods et al. demonstrated that $\mathrm{AT}_{1-}$ receptor blockade during the first 12 days of neonatal life resulted in a reduced number of glomeruli ${ }^{189}$. They also found that perinatal protein restriction in the rat suppresses the newborn intrarenal renin-angiotensin system and leads to a decreased glomerular number ${ }^{82}$. The exact mechanisms underlying the renal abnormalities as a result of a lack of $A T_{1}$ receptor stimulation are still unclear, but may involve disturbed cell-cell and cell-matrix interactions and disbalance between proliferation and apoptosis due to altered expression of various growth factors which are important for kidney development ${ }^{188}$. Although day 13 of pregnancy might be a too early time point to alter nephrogenesis, it does not imply that adult renal function cannot be modified by the altered arterial adrenergic reactivity during development. As mentioned above, $\alpha$ - and $\beta$ adrenoceptors are involved in the regulation of a variety of renal functions, like the renal renin-angiotensin system and a normal development of renal adrenoceptor function seems to be important for normal kidney function in the adult. Further experiments are necessary to investigate the consequences of an early induction of IUS for adult renal function. 
The changes in glomerular properties that we observed in young rats that survived IUS induced at day 17 of pregnancy, persisted throughout adulthood. We also found that the reduced number of glomeruli (reduced filtration surface area) led to the development of glomerular hypertrophy. hyperfiltration and an increased renal blood flow and filtration fraction in the adult (chapter 4). This occurs most likely to compensate for the decreased glomerular density and to achieve an adequate renal function, as indicated by the normal plasma creatinine concentrations. On the other hand, Thomson et al. propose a hypothesis that suggests that another mechanism could be responsible for the increased glomerular filtration rate (GFR). This hypothesis is based on findings in diabetic rats and humans ${ }^{173}$. Diabetes affects the kidney in stages and at the very onset of diabetes, the kidney grows large and the GFR becomes supranormal ${ }^{169}$. The factors that determine GFR are directly influenced by various effectors that include nerves, hormones, size and condition of the glomeruli and tubuloglomerular feedback (TGF) from the macula densa. Physiological effectors of GFR generally function as elements in negative feedback systems that link GFR to other physiological parameters. GFR is linked to extracellular fluid volume through multiple parallel feedback loops that incorporate the renal nerves, renin-angiotensin system and natriuretic peptides and changes to elements of this loop are referred to as primary vascular events. GFR is also linked to the concentration of sodium in the tubular fluid that reaches the macula densa by TGF and changes to elements of this loop are referred to as primary tubular events. The feedback loops that link GFR to extracellular volume compete for control of GFR with the loop that links GFR to macula densa sodium. Thomson et al. propose that tubular hypertrophy increases reabsorption in the proximal tubules and less sodium reaches the macula densa. This in turn increases GFR via TGF. Simply stated, tubular hypertrophy causes glomerular hyperfiltration via hyper-reabsorption in diabetes ${ }^{173.175}$. Whether this mechanism is involved in the renal structural and functional changes induced by IUS is not clear, but it is certainly an interesting hypothesis to include in future experiments.

In summary, factors that affect nephrogenesis in the fetus are not only critical in kidney devellopment but also affect subsequent adult renal function. Further evidence for a challenged renal function is the development of albuminuria as a result of IUS. Albuminuria is probably the result of an increased intraglomerular pressure associated with hyperfiltration and predicts an increased risk for cardiovascular disease and renal functional abnormalities ${ }^{190}$. Glomerular hypertrophy and hyperfiltration are often predictors of glomerular damage and progression of glomerular sclerosis ${ }^{191}$. However, we could not detect any evidence of glomerular sclerosis. The age of 6 month might be too young to observe microscopically visible indications of glomerular pathology. 
It has been proposed that a reduced nephron endowment at birth results in the development of hypertension in the adult ${ }^{15}$. However, even though we observed a reduced glomerular density, we could not demonstrate an increase in blood pressure. In humans, hypertension is a pathological condition that generally does not become apparent until middle age. Again, more time may have been needed for hypertension to develop in our experimental animals. The $30 \%$ reduction in glomerular number and the subsequent glomerular hypertrophy and hyperfiltration did not induce hypertension under standard conditions. We therefore evaluated the effects of unilateral nephrectomy and high sodium intake.

\subsection{Effects of unilateral nephrectomy and high sodium intake after IUS}

After an additional reduction in the number of glomeruli by unilateral nephrectomy at 21 days of age, we observed an increase in blood pressure in adult animals that survived IUS. Furthermore, IUS and additional nephrectomy resulted in severe renal insufficiency, as indicated by high plasma creatinine concentrations, and a reduced renal compensatory capacity in the adult offspring. An acquired nephron deficit after surgical reduction of renal mass does not always lead to the development of hypertension, unless the nephrectomy is performed just after birth ${ }^{192.193}$ or unless kidney donors have underlying conditions such as obesity or diabetes $^{194,195}$. The reduced filtration surface area as a result of the reduced nephron endowment observed in our experiments seems to be an underlying condition in which a further reduction of renal mass results in hypertension. Additionally, the kidneys are incapable of adapting renal function to a further reduction of the nephron number and therefore start to fail.

Also treatment of the animals with a high sodium diet resulted in an increase in blood pressure. Again we found that IUS animals had lost their ability to adapt renal function to an additional challenge such as a high sodium diet. High sodium itself has a negative effect on renal function, because it increases glomerular filtration rate and the filtration fraction. IUS animals already have an increased glomerular filtration rate (GFR), renal blood flow and filtration fraction. A further increase of these parameters as a consequence of the high sodium intake seemed to be impossible and as a result the kidneys failed. Although the mechanism of the altered glomerular hemodynamics induced by high sodium is not fully clear, neuroendocrine systems like the renin-angiotensin system ${ }^{176,177}$, the sympathetic nervous system $^{178}$, or a deficiency of vasodilator substances like nitric oxide ${ }^{196}$ seem to be responsible for the vasoconstriction of the efferent glomerular arterioles $^{163}$. The same systems also seem to be important in the fetal 
programming of cardiovascular disease ${ }^{3,6-6,196}$ and might be involved in the altered renal function that we observed in animals that survived IUS. These animals are perhaps incapable of adlapting these systems to a further challenge with high sodium and develop renal failure, as indicated by the high urinary albumin concentrations. Additionally, high sodium can be harmful to the selective permeability of the glomerular basement membrane ${ }^{197}$ and worsen the urinary excretion of albumin.

Another possible mechanism that could be involved in the reduced GFR in sodium treated rats that survived IUS, is postulated by Thomson and colleagues ${ }^{173,175}$. In early diabetes, kidney growth causes an increase in proximal reabsorption and the proximal tubules also assume a major role in sodium balance, leading to a paradoxical effect of dietary sodium on GFR. This paradox arises in diabetes because the proximal tubule is more sensitive to sodium intake, making GFR a "slave" to tubular function. In contrary to what one would expect, Vallon et al. found that high sodium intake decreased GFR in diabetic rats. They observed a prominent negative impact of dietary salt on reabsorption upstream from the macula densa and a major decrease in proximal reabsorption ${ }^{198}$. This occurs because diabetes causes proximal tubular reabsorption to become more sensitive to changes in dietary salt. This results in a strong influence of dietary salt on the tubuloglomerular feedback (TGF) signal such that ingesting more sodium leads to greater activation of TGF and vice versa. However, the mechanism that makes the diabetic proximal tubule more sensitive to dietary sodium remains to be determined.

Altogether, the hypothesis postulated by Thomson et al. might also be relevant for the altered renal function we observed as a consequence of IUS. IUS might lead to tubular hypertrophy and an increased reabsorption of the proximal tubules, which makes these tubules more sensitive to dietary sodium intake.

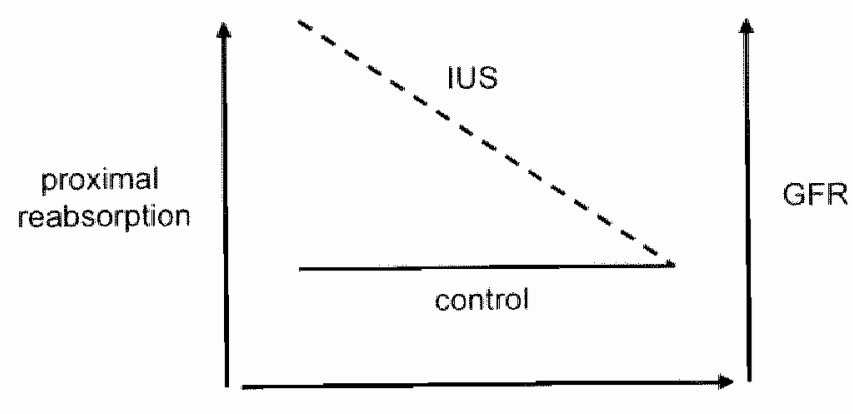

$\mathrm{NaCl}$ intake

Figure 6.1 Schematic presentation of the relationship between sodium intake and reabsorption in the proximal tubules as well as GFR. (adapted from Vallon et all. ${ }^{175}$ ) 
Besides influencing renal function, high sodium loading can also lead to the development of target organ damage, like left ventricular hypertrophy and renal fibrosis. We could not observe any changes in left ventricular wall mass or any visible signs of glomerulosclerosis: Either the age of the animals was too young to develop left ventricular hypertrophy and collagen deposition in the kidney, or the treatment with high sodium was not extensive enough to induce organ damage. Alternatively it has been reported that sodium sensitive patients can develop impaired left ventricular diastolic function without any differences in left ventricular wall mass ${ }^{185}$. This implies that IUS animals might acquire left ventricular dysfunction after treatment with a high sodium diet.

\subsection{Perspectives}

Data, put forward by Barker, on the inverse relationship between blood pressure and birth weight and the possible mechanisms underlying this phenomenon suggest an epigenetic effect of perinatal factors on the development of cardiovascular disease in the adult. This effect is referred to as the "Barker hypothesis" or as "perinatall programming of adult disease" . Another hypothesis was postulated by Brenner; maladaptive hemodynamic changes lead to glomerular hyperfiltration, an adaptation seen in response to a reduction in functional nephron number ${ }^{14}$.

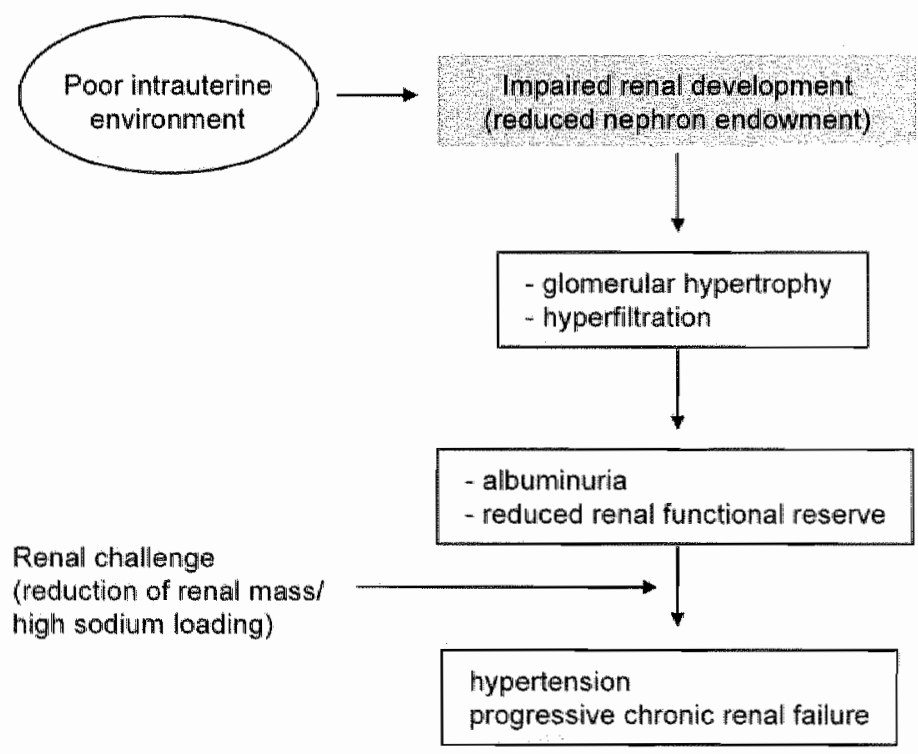

Figure 6.2 Unification of the Barker and Brenner hypothesis. 
The present thesis provides evidence that "Barker meets Brenner" and that the two hypotheses can be unified; a poor uterine environment, reflected by low birth weight or more appropriately by increased hematocrit values, results in fewer nephrons and a lower renal functional reserve (iig, 6.2).

A more thorough analysis of the systems involved in the altered renal function due to IUS is required to fully appreciate the consequences for the development of cardiovascular disease later in life. In humans, cardiovascular diseases usually do not become apparent until middle age. For that reason it would be desirable to perform studies in older rats in an attempt to provide a better impression of the effects of IUS on blood pressure and renal structure.

Since several intrauterine risk factors for low birth weight and low nephron number are alleady known, it is certainly important to avoid these factors during pregnancy. In the near future it will become important for paediatricians to identify children at higher risk for cardiovascular disease early in life in order to monitor and treat them adequately. The latter will require pharmaco-therapy aimed at optimising renal function in the setting of reduced number of enlarged glomeruli and tubuli. 


\section{References}

1. Barker DJ. In utero programming of cardiovascular disease. Theriogenology. 2000;53:555-574.

2. Hoet $\mathrm{JJ}$, Hanson MA. Intrauterine nutrition: its importance during critical periods for cardiovascular and endocrine development. $J$ Physiol. 1999:514 (Pt 3):617-627.

3. Ruijtenbeek K, le Noble FA, Janssen GM, Kessels CG, Fazzi GE, Blanco $C E$, De Mey JG. Chronic hypoxia stimulates periarterial sympathetic nerve development in chicken embryo. Circulation. 2000;102:2892-2897.

4. Lueder FL, Kim SB, Buroker CA, Bangalore SA, Ogata ES. Chronic maternal hypoxia retards fetal growth and increases glucose utilization of select fetal tissues in the rat. Metabolism. 1995;44:532537.

5. Wigglesworth JS. Experimental Growth Retardation in the Foetal Rat. J Pathol Bacteriol. 1964;88:1-13.

6. Jansson $T$, Lambert GW. Effect of intrauterine growth restriction on blood pressure, glucose tolerance and sympathetic nervous system activity in the rat at 3-4 months of age. J Hypertens. 1999;17:12391248 .

7. Green LR. Programming of endocrine mechanisms of cardiovascular control and growth. J Soc Gynecol Investig. 2001;8:57-68.

8. Holemans K, Gerber R, Meurrens K, De Clerck F, Poston L, Van Assche FA. Maternal food restriction in the second half of pregnancy affects vascular function but not blood pressure of rat female offspring. Br J Nutr. 1999;81:73-79.

9. Marchand MC, Langley-Evans SC. Intrauterine programming of nephron number: the fetal flaw revisited. J Nophrol. 2001;14:327331.

10. Moritz KM, Dodic M, Wintour EM. Kidney development and the fetal programming of adult disease. Bioessays. 2003;25:212-220.

11. Lee RM. Vascular changes at the prehypertensive phase in the mesenteric arteries from spontaneously hypertensive rats. Blood Vessels. 1985;22:105-126.

12. Head RJ. Hypemoradrenergic innervation: its relationship to functional and hyperplastic changes in the vasculature of the spontaneously hypertensive rat. Blood Vessels. 1989;26:1-20.

13. Slotkin TA, Auman JT, Seidler FJ. Ontogenesis of betaadrenoceptor signaling: implications for perinatal physiology and for fetal effects of tocolytic drugs. J Pharmacol Exp Ther. 2003;306:1-7.

14. Brenner BM, Lawler EV, Mackenzie HS. The hyperfiltration theory: a paradigm shift in nephrology. Kidney Int. 1996;49:1774-1777. 
15. Brenner $\mathrm{BM}_{3}$ Garcia DL, Anderson S. Glomeruli and blood pressure. Less of one, more the other? Am J Hypertens. 1988;1:335-347.

16. Carmeliet $P$. Mechanisms of angiogenesis and arteriogenesis. Nat Med. 2000;6:389-395.

17. Gomez RA, Norwood VF, Tufro-McReddie A. Development of the kidney vascislature. Microsc Res Tech. 1997;39:254-260.

18. Hungerford JE, Little CD. Developmental biology of the vascular smooth muscle cell: building a multilayered vessel wall. J Vasc Res. $1999 ; 36: 2-27$.

19. Langille $\mathrm{BL}$. Remodeling of developing and mature arteries: endothelium, smooth muscle, and matrix. J Cardiovasc Pharmacol. 1993;21 Suppl 1:S11-17.

20. Pourageaud F, De Mey JG. Structural properties of rat mesenteric small arteries after 4-wk exposure to elevated or reduced blood flow. Am J Physiol. 1997;273:H1699-1706.

21. Buus $C L$, Pourageaud $F$, Fazzi GE, Janssen $G$, Mulvany $M J, D e$ Mey JG. Smooth muscle cell changes during flow-related remodeling of rat mesenteric resistance arteries. Circ Res. 2001;89:180-186.

22. Langille BL. Arterial remodeling: relation to hemodynamics. Can J Physiol Pharmacol. 1996;74:834-841.

23. Stenmark $K$, Weiser $M$, Buga $G$, Ignarro L. Vascular development and function. In: Gluckman $P$, Heysmann $M$, eds. Pediatrics and perinatology. New York: Arnold; 1996:681-709.

24. Daemen MJ, De Mey JG. Regional heterogeneity of arterial structural changes. Hypertension. 1995;25:464-473.

25. Stassen FR, Willemsen MJ, Janssen GM, DeMey JG. Alpha 1adrenoceptor subtypes in rat aorta and mesenteric small arteries are preserved during left ventricular dysfunction post-myocardial infarction. Cardiovasc Res. 1997;33:706-713.

26. Francis NJ, Landis SC. Cellular and molecular determinants of sympathetic neuron development. Annu Rev Neurosci. 1999;22:541566.

27. Falckh PH, Harkin LA, Head RJ. Nerve growth factor mRNA content parallels altered sympathetic innervation in the spontaneously hypertensive rat. Clin Exp Pharmacol Physiol. 1992;19:541-545.

28. Falckh $\mathrm{PH}$, Harkin LA, Head RJ. Resistance vessel gene expression of nerve growth factor is elevated in young spontaneously hypertensive rats. J Hypertens. 1992;10:913-918.

29. Aberdeen J, Corr L, Milner P, Lincoln J, Burnstock G. Marked increases in calcitonin gene-related peptide-containing nerves in the developing rat following long-term sympathectomy with guanethidine. Neuroscience. 1990;35:175-184.

30. Ralevic $V$, Karoon $P$ "Burnstock $G$. Long-term sensory denervation by neonatal capsaicin treatment augments sympathetic neurotransmission in rat mesenteric arteries by increasing levels of 
norepinephrine and selectively enhancing postjunctional actions. $J$ Pharmacol Exp Ther. 1995;274:64-71.

31. Berridge MJ. Elementary and global aspects of calcium signalling. $J$ Physiol. 1997;499 (Pt 2):291-306.

32. Berridge MJ. Inositol trisphosphate and calcium mobilization. $J$ Cardiovasc Pharmacol. 1986;8 Suppl 8:S85-90.

33. Berridge MJ. Cell signaling through phospholipid metabolism. $J$ Cell Sici Suppl. 1986;4:137-153.

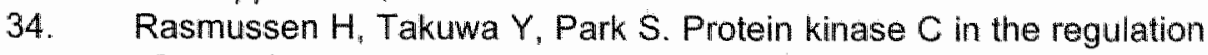
of smooth muscle contraction. Faseb J. 1987;1:177-185.

35. Adelstein RS, Sellers JR. Effects of calcium on vascular smooth muscle contraction. Am J Cardiol. 1987;59:4B-10B.

36. Somlyo AP. Somlyo AV. Signal transduction by G-proteins, rhokinase and protein phosphatase to smooth muscle and non-muscle myosin II. J Physiol. 2000;522 Pt 2:177-185.

37. Artalejo CR, Ariano MA, Perlman RL, Fox AP. Activation of facilitation calcium channels in chromaffin celis by $D 1$ dopamine receptors through a cAMP/protein kinase A-dependent mechanism. Nature. 1990;348:239-242.

38. Heesen BJ, De Mey JG. Effects of cyclic AMP-affecting agents on contractile reactivity of isolated mesenteric and renal resistance arteries of the rat. Br J Pharmacol. 1990;101:859-864.

39. Birnbaumer $L$. $G$ proteins in signal transduction. Annu Rev Pharmacol Toxicol. 1990;30:675-705.

40. Moncada S, Higgs EA. Endogenous nitric oxide: physiology, pathology and clinical relevance. Eur J Clin Invest. 1991;21:361-374.

41. Triggle $C R$, Hollenberg $M$, Anderson $T J$, Ding $H$, Jiang $Y$, Ceroni $L$, Wiehler WB, $\mathrm{Ng}$ ES, Ellis A, Andrews K, McGuire JJ, Pannirselvam $M$. The endothelium in health and disease--a target for therapeutic intervention. J Smooth Muscle Res. 2003;39:249-267.

42. Bell D, McDermott BJ. Calcitonin gene-related peptide in the cardiovascular system: characterization of receptor populations and their (patho)physiological significance. Pharmacol Rev. 1996;48:253-288.

43. Guimaraes S, Moura D. Vascular adrenoceptors: an update. Pharmacol Rev. 2001;53:319-356.

44. Schlaich MP, Lambert E, Kaye DM, Krozowski Z, Campbell DU, Lambert G, Hastings J, Aggarwal A, Esler MD. Sympathetic augmentation in hypertension: role of nerve firing, norepinephrine reuptake, and Angiotensin neuromodulation. Hypertension. 2004:43:169-175.

45. Mancia G, Grassi G, Giannattasio C, Seravalle G. Sympathetic activation in the pathogenesis of hypertension and progression of organ damage. Hypertension. 1999;34:724-728.

46. Clark AT, Bertram JF. Advances in renal development. Curr Opin Nephrol Hypertens. 2000;9:247-251. 
47. Moritz KM, Wintour EM. Functional development of the meso- and metanephros. Pediatr Nephrol. 1999;13:171-178.

48. Guron G, Friberg P. An intact renin-angiotensin system is a prerequisite for normal renal development. I Hyperiens. 2000;18:123-137.

49. Guyton A, Hall J. Textbook of medical physiology. Philadelphia: W.B. Saunders Company; 2000.

50. Levey AS. Measurement of renal function in chronic renal disease. Kidney Int. 1990;38:167-184.

51. Maack T. Role of atrial natriuretic factor in volume control. Kidney Int. 1996;49:1732-1737.

52. Sarnak MJ, Levey AS, Schoolwerth AC, Coresh J, Culleton $B$, Hamm LL, McCullough PA, Kasiske BL, Kelepouris E, Klag MJ,

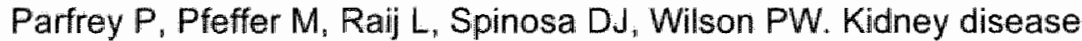
as a risk factor for development of cardiovascular disease: a statement from the American Heart Association Councils on Kidney in Cardiovascular Disease, High Blood Pressure Research, Clinical Cardiology, and Epidemiology and Prevention. Hypertension. 2003;42:1050-1065.

53. Brenner BM, Mackenzie HS. Nephron mass as a risk factor for progression of renal disease. Kidney Int Suppl. 1997;63:S124-127.

54. Mackenzie HS, Brenner BM. Fewer nephrons at birth: a missing link in the etiology of essential hypertension? Am J Kidney Dis. $1995 ; 26: 91-98$.

55. Mimran $A_{1}$ Ribstein $J$, Du Cailar $G$. Microalbuminuria in essential hypertension. Curr Opin Nephrol Hypertens. 1999;8:359-363.

56. Osmond C, Barker DJ. Fetal, infant, and childhood growth are predictors of coronary heart disease, diabetes, and hypertension in adult men and women. Environ Health Perspect. 2000;108 Suppl 3:545-553.

57. Godfrey KM, Barker DJ. Fetal nutrition and adult disease. Am J Clin Nutr. 2000;71:1344S-1352S.

58. Leon D, Koupilova I. Birth weight, blood pressure, and hypertension: Epidemiological studies. In: Barker $D_{0}$ ed. Fetal origins of cardiovascular and lung disease. 1 ed. New York: Marcel Dekker Inc.; 2001:23-48.

59. Huxley $R$, Neil $A$, Collins R. Unravelling the fetal origins hypothesis: is there really an inverse association between birthweight and subsequent blood pressure? Lancet. 2002;360:659-665.

60. Miller SL, Green LR, Peebles DM, Hanson MA, Blanco CE. Effects of chronic hypoxia and protein malnutrition on growth in the developing chick. Am J Obstet Gynecol. 2002;186:261-267.

61. Kollee LA, Monnens LA, Trijbels JM. Veerkamp JH, Janssen AJ. Experimental intrauterine growth retardation in the rat. Evaluation of the Wigglesworth model. Early Hum Dev. 1979;3:295-300. 
62. Cock ML. Camm EJ, Louey S, Joyce BJ, Harding R. Postnatal outcomes in term and preterm lambs following fetal growth restriction. Clin Exp Pharmacol Physiol. 2001;28:931-937.

63. Roseboom TJ, van der Meulen JH, Ravelli AC, Osmond $C$, Barker DJ, Bleker OP. Effects of prenatal exposure to the Dutch famine on adult disease in later life: an overview. Mol Cell Endocrinol. 2001;185:93-98.

64. Leon DA, Koupilova I, Lithell HO, Berglund L, Mohsen R, Vagero D, Lithell UB, McKeigue PM. Failure to realise growth potential in utero and adult obesity in relation to blood pressure in 50 year old Swedish men. Bmj. 1996:312:401-406.

65. Eriksson JG, Forsen T, Tuomilehto J, Winter PD, Osmond C, Barker DJ. Catch-up growth in childhood and death from coronary heart disease: longitudinal study. Bmj. 1999;318:427-431.

66. Eriksson J, Forsen T, Tuomilehto J, Osmond C, Barker D. Fetal and childhood growth and hypertension in adult life. Hypertension. 2000;36:790-794.

67. Hales CN, Ozanne SE. For debate: Fetal and early postnatal growth restriction lead to diabetes, the metabolic syndrome and renal failure. Diabetologia. 2003;46:1013-1019.

68. Moore LG, Shriver M, Bemis $L$, Hickler B, Wilson M, Brutsaert $T$, Parra $E_{\text {, Vargas }} E$. Maternal adaptation to high-altitude pregnancy: an experiment of nature--a review. Placenta. 2004;25 Suppl A:S6071.

69. Li G, Xiao Y, Estrella JL, Ducsay CA, Gilbert RD, Zhang L. Effect of fetal hypoxia on heart susceptibility to ischemia and reperfusion injury in the adult rat. I Soc Gynecol Investig. 2003;10:265-274.

70. Ruijtenbeek K, Kessels LC, De Mey JG, Blanco CE. Chronic moderate hypoxia and protein malnutrition both induce growth retardation, but have distinct effects on arterial endotheliumdependent reactivity in the chicken embryo. Pediatr Res. 2003;53:573-579.

71. Head RJ. Hypernoradrenergic innervation and vascular smooth muscle hyperplastic change. Blood Vessels. 1991;28:173-178.

72. IJzerman $R$, Stehouwer $C D$, de Geus $E J$, van Weissenbruch MM, Delemarre-van de Waal HA, Boomsma DI. Low birth weight is associated with increased sympathetic activity: dependence on genetic factors. Circulation. 2003;108:566-571.

73. Goodfellow J, Bellamy MF, Gorman ST, Brownlee M, Ramsey MW, Lewis MJ, Davies DP, Henderson AH. Endothelial function is impaired in fit young adults of low birth weight. Cardiovasc Res. 1998;40:600-606.

74. Merlet-Benichou C, Gilbert T, Muffat-Joly M, Lelievre-Pegorier M, Leroy $B$. Intrauterine growth retardation leads to a permanent nephron deficit in the rat. Pediatr Nephrol. 1994;8:175-180. 
75. Woods LL. Fetal origins of adult hypertension: a renal mechanism? Curr Opin Nephrol Hypertens. 2000:9:419-425.

76. Nwagwu MO, Cook A, Langley-Evans SC. Evidence of progressive deterioration of renal function in rats exposed to a maternal lowprotein diet in utero. Br J Nutr. 2000;83:79-85.

77. Manning J, Vehaskari VM. Low birth weight-associated adult hypertension in the rat. Pediatr Nephrol. 2001:16:417-422.

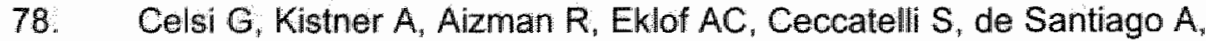
Jacobson SH. Prenatal dexamethasone causes oligonephronia, sodium retention, and higher blood pressure in the offspring. Pediatr Res. 1998;44:317-322.

79. Seckl JR, Cleasby M. Nyirenda MJ. Glucocorticoids, 11betahydroxysteroid dehydrogenase, and fetal programming. Kidney Int. $2000 ; 57: 1412-1417$.

80. Kornel $L$, Prancan AV, Kanamarlapudi $N$, Hynes J, Kuzianik E. Study on the mechanisms of glucocorticoid-induced hypertension: glucocorticoids increase transmembrane $\mathrm{Ca} 2+$ influx in vascular smooth muscle in vivo. Endocr Res. 1995;21:203-210.

81. Langley-Evans SC. Intrauterine programming of hypertension by glucocorticoids. Life Sci. 1997;60:1213-1221.

82. Woods LL, Ingelfinger JR, Nyengaard JR, Rasch R. Maternal protein restriction suppresses the newborn renin-angiotensin system and programs adult hypertension in rats. Pediatr Res. 2001;49:460-467.

83. Vehaskari VM, Aviles $\mathrm{DH}$, Manning J. Prenatal programming of adult hypertension in the rat. Kidney Int. 2001;59:238-245.

84. Tufro-McReddie A, Romano LM, Harris JM. Ferder L, Gomez RA. Angiotensin 11 regulates nephrogenesis and renal vascular development. Am J Physiol. 1995;269:F110-115.

85. do Carmo Pinho Franco $M$, Nigro $D$, Fortes $Z B$, Tostes $R C$, Carvalho MH, Lucas SR, Gomes GN, Coimbra TM, Gill FZ. Intrauterine undernutrition-renal and vascular origin of hypertension. Cardiovasc Res. 2003;60:228-234.

86. Franco Mdo C, Arruda RM, Dantas AP, Kawamoto EM, Fortes ZB, Scavone C, Carvalho MH, Tostes RC. Nigro D. Intrauterine undernutrition: expression and activity of the endothelial nitric oxide synthase in male and female adult offspring. Cardiovasc Res. 2002;56:145-153.

87. Brawley $L_{*}$ Itoh $S$, Torrens C, Barker A, Bertram C, Poston L, Hanson M. Dietary protein restriction in pregnancy induces hypertension and vascular defects in rat male offspring. Pediatr Res. 2003;54:83-90.

88. Lamireau D, Nuyt AM, Hou X, Bernier S, Beauchamp M, Gobeil F, Jr., Lahaie I, Varma DR, Chemtob $S$. Altered vascular function in fetal programming of hypertension. Stroke. 2002;33:2992-2998.

89. Franco Mdo C, Dantas AP, Akamine EH, Kawamoto EM, Fortes ZB, Scavone C, Tostes RC, Carvalho MH, Nigro D. Enhanced oxidative 
stress as a potential mechanism underlying the programming of hypertension in utero. J Cardiovasc Pharmacol. 2002;40:501-509.

90. Murotsuki J, Bocking AD, Gagnon R. Fetal heart rate patterns in growth-restricted fetal sheep induced by chronic fetal placental embolization. Am J Obstet Gynecol. 1997;176:282-290.

91. Lang U, Baker RS, Braems $G$, Zygmunt M, Kunzel W, Clark KE. Uterime blood flow--a determinant of fetal growth. Eur $J$ Obstet Gynecol Reprod Biol. 2003;110 Suppl 1:S55-61.

92. Murotsuki J, Gagnon $R, P u X$, Yang $K$. Chronic hypoxemia selectively down-regulates 11 beta-hydroxysteroid dehydrogenase type 2 gene expression in the fetal sheep kidney. Biol Reprod. 1998:58:234-239.

93. Martyn CN, Barker DJ, Jespersen S, Greenwald S, Osmond C, Berry $C$. Growth in utero, adult blood pressure, and arterial compliance. Br Heart J. 1995;73:116-121.

94. Gagnon R. Placental insufficiency and its consequences. Eur $J$ Obstet Gynecol Reprod Biol. 2003;110 Suppl 1:S99-107.

95. Alexander BT. Placental insufficiency leads to development of hypertension in growth-restricted offspring. Hypertension. 2003:41:457-462.

96. Simmons RA, Templeton LJ, Gertz SJ. Intrauterine growth retardation leads to the development of type 2 diabetes in the rat. Diabetes. 2001;50:2279-2286.

97. Bevan RD. Influence of adrenergic innervation on vascular growth and mature characteristics. Am Rev Respir Dis. 1989;140:14781482.

98. Bevan J, Bevan R, Duckles S. Adrenergic regulation of vascular smooth muscle. In: Bohr D, Somlyo A, Sparks H, eds. Handbook of physiology. Bethesta: American Physiological Society; 1980:515565.

99. Jones CT, Roebuck MM, Walker DW, Johnston BM. The role of the adrenal medulla and peripheral sympathetic nerves in the physiological responses of the fetal sheep to hypoxia. J Dev Physiol. 1988;10:17-36.

100. Cochard $\mathrm{P}$, Goldstein M, Black IB. Ontogenetic appearance and disappearance of tyrosine hydroxylase and catecholamines in the rat embryo. Proc Natl Acad Sci U S A. 1978;75:2986-2990.

101. Jonakait GM, Wolf J Cochard P, Goldstein M, Black IB. Selective loss of noradrenergic phenotypic characters in neuroblasts of the rat embryo. Proc Natl Acad Sci U S A. 1979;76:4683-4686.

102. Robinson RB. Autonomic receptor--effector coupling during postnatal development. Cardiovasc Res. 1996;31 Spec No:E68-76.

103. De Mey JG, Brutsaert DL. Mechanical properties of resting and active isolated coronary arteries. Circ Res. 1984;55:1-9.

104. Bulbring $E$, Tomita T. Catecholamine action on smooth muscle. Pharmacol Rev. 1987;39:49-96. 
105. Han SP, Naes L, Westfall TC. Calcitonin gene-related peptide is the endogenous mediator of nonadrenergic-noncholinergic vasodilation in rat mesentery. J Pharmacol Exp Ther. 1990;255:423-428.

106. Sanders M, van Eyndhoven H, Fazzi G, De Mey J. Relaxing responses to CGRP only in arteries equipped with CGRP-containing nerves. Unpublished data. 2002.

107. Holzer P. Capsaicin: cellular targets, mechanisms of action, and selectivity for thin sensory neurons. Pharmacol Rev. 1991;43:143201.

108. Szallasi A, Blumberg PM. Vanilloid (Capsaicin) receptors and mechanisms. Pharmacol Rev. 1999;51:159-212.

109. Cohen RA, Vanhoutte PM. Endothelium-dependent hyperpolarization. Beyond nitric oxide and cyclic GMP. Circulation. 1995;92:3337-3349.

110. Stassen FR, Raat $N J$, Brouwers-Ceiler DL, Fazzi GE, Smits JF, De Mey JG. Angiotensin II induces media hypertrophy and hyperreactivity in mesenteric but not epigastric small arteries of the rat. J Vasc Res. 1997;34:289-297.

111. Mills $E$, Smith PG. Mechanisms of adrenergic control of blood pressure in developing rats. Am J Physiol. 1986;250:R188-192.

112. Su C, Bevan JA, Assali NS, Brinkman CR, 3rd. Development of neuroeffector mechanisms in the carotid artery of the fetal lamb. Blood Vessels. 1977;14:12-24.

113. Pappano AJ, Loffelholz $K$. Ontogenesis of adrenergic and cholinergic neuroeffector transmission in chick embryo heart. $J$ Pharmacol Exp Ther. 1974;191:468-478.

114. Stassen FR, Maas RG, Schiffers PM, Janssen GM, De Mey JG. A positive and reversible relationship between adrenergic nerves and alpha-1A adrenoceptors in rat arteries. I Pharmacol Exp Ther. 1998:284:399-405.

115. Supowit SC, Hallman DM, Zhao H, DiPette DJ. Alpha 2-adrenergic receptor activation inhibits calcitonin gene-related peptide expression in cultured dorsal root ganglia neurons. Brain Res. 1998;782:184-193.

116. Vanhoutte PM, Verbeuren TJ, Webb RC. Local modulation of adrenergic neuroeffector interaction in the blood vessel well. Physiol Rev. 1981;61:151-247.

117. Hill CE, Phillips JK, Sandow SL. Development of peripheral autonomic synapses: neurotransmitter receptors, neuroeffector associations and neural influences. Clin Exp Pharmacol Physiol. $1999 ; 26: 581-590$.

118. Franco-Cereceda A, Rudehill A, Lundberg JM. Calcitonin generelated peptide but not substance $P$ mimics capsaicin-induced coronary vasodilation in the pig. Eur $J$ Pharmacol. 1987;142:235243. 
119. Kilts JD, Gerhardt MA, Richardson MD, Sreeram G, Mackensen GB, Grocott HP, White WD, Davis RD, Newman MF, Reves JG, Schwinn $D A$, Kwatra MM. Beta(2)-adrenergic and several other $G$ proteincoupled receptors in human atrial membranes activate both $\mathrm{G}(\mathrm{s})$ and $\mathrm{G}(\mathrm{i})$. Circ Res. 2000;87:705-709.

120. Derweesh $H$, Wheeler MA, Weiss RM. Alterations in G-proteins and beta-adrenergic responsive adenylyl cyclase in rat urinary bladder during aging. J Phamacol Exp Ther, 2000;294:969-974.

121. Ludford $\mathrm{JM}$, Talamo $\mathrm{BR}$. Independent regulation of betasadrenergic receptor and nucleotide binding proteins of adenylate cyclase. Developmental and denervation-dependent responses in rat parotid. $J$ Biol Chem. 1983;258:4831-4838.

122. Hausdorff WP, Caron MG, Lefkowitz RJ. Turning off the signal: desensitization of beta-adrenergic receptor function. Faseb $\mathrm{J}$. 1990;4:2881-2889.

123. Slotkin TA, Lau $C$, Seidler FJ. Beta-adrenergic receptor overexpression in the fetal rat: distribution, receptor subtypes, and coupling to adenylate cyclase activity via G-proteins. Toxicol App/ Pharmacol. 1994;129:223-234.

124. Auman JT, Seidler FJ, Slotkin TA. Regulation of fetal cardiac and hepatic beta-adrenoceptors and adenylyl cyclase signaling: terbutaline effects. Am J Physiol Regul Integr Comp Physiol. 2001;281:R1079-1089.

125. Lagercrantz $H$, Bistoletti $P$. Catecholamine release in the newborn infant at birth. Pediatr Res. 1977;11:889-893.

126. De Mey JG, Dijkstra EH, Vrijdag MJ. Endothelium reduces DNA synthesis in isolated arteries. Am J Physiol. 1991;260:H1128-1134.

127. Pardi $G$, Marconi AM, Cetin I. Placental-fetal interrelationship in IUGR fetuses--a review. Placenta. 2002;23 Suppl A:S136-141.

128. Tsuru $H$, Tanimitsu N, Hirai $T$. Role of perivascular sympathetic nerves and regional differences in the features of sympathetic innervation of the vascular system. Jpn J Pharmacol. 2002;88:9-13.

129. Satoh M, Enomoto K, Takayanagi I, Koike K. Analysis of alpha1. adrenoceptor subtypes in rabbit aorta and arteries: regional difference and co-existence. Eur J Pharmacol. 1999;374:229-240.

130. Lucas SR, Costa Silva VL, Miraglia SM, Zaladek Gil F. Functional and morphometric evaluation of offspring kidney after intrauterine undernutrition. Pediatr Nephrol. 1997;11:719-723.

131. Michel MC, Buscher R, Philipp T, Brodde OE. Alpha $1 \mathrm{~A}$ and alpha $1 \mathrm{~B}$-adrenoceptors enhance inositol phosphate generation in rat renal cortex. Naunyn Schmiedebergs Arch Pharmacol. 1993;347:180-185.

132. Barker D. Fetal origins of cardiovascular and lung disease. Basel: Marcel Dekker Inc.; 2001.

133. Adabag AS. Birthweight and the future risk of cardiovascular disease: does intrauterine malnutrition have a role in fetal programming? J Lab Clin Med. 2001;138:378-386. 
134. Engelbregt MJ, van Weissenbruch MM, Popp-Snijders $C$, Delemarre-van de Waal HA. Delayed first cycle in intrauterine growth-retarded and postnatally undernourished female rats: follicular growth and ovulation after stimulation with pregnant mare serum gonadotropin at first cycle. J Endocrinol. 2002;173:297-304.

135. van der Loop FT, Gabbiani G, Kohnen G, Ramaekers FC, van Eys GJ. Differentiation of smooth muscle cells in human blood vessels as defined by smoothelin, a novel marker for the contractile phenotype. Arterioscler Thromb Vasc Biol. 1997;17:665-671.

136. Wolff DW, Gesek FA, Strandhoy JW. In vivo assessment of rat renal alpha adrenoceptors. J Pharmacol Exp Ther. 1987;241:472-476.

137. Uchino $K$, Frohlich $E D$, Nishikimi $T$, Isshiki $T$, Kardon MB. Spontaneously hypertensive rats demonstrate increased renal vascular alpha 1-adrenergic receptor responsiveness. Am J Physiol. 1991;260:R889-893.

138. Azar S, Johnson MA, Scheinman J, Bruno L, Tobian L. Regulation of glomerular capillary pressure and filtration rate in young Kyoto hypertensive rats. Clin Sci (Lond). 1979;56:203-209.

139. Vanhoutte $P$, Amery A, Birkenhager W, Breckenridge A, Buhler $F$, Distler A, Dormandy J, Doyle $A$, Frohlich E, Hansson $L$, et al. Serotoninergic mechanisms in hypertension. Focus on the effects of ketanserin. Hypertension. 1988;11:111-133.

140. Boston PC, Hodgson WC. Changes in reactivity towards 5hydroxytryptamine in the renal vasculature of the diabetic spontaneously hypertensive rat. J Hypertens. 1997;15:769-774.

141. Ozaki $T$, Nishina $H$, Hanson MA, Poston L. Dietary restriction in pregnant rats causes gender-related hypertension and vascular dysfunction in offspring. J Physiol. 2001;530:141-152.

142. Werstiuk ES, Lee RM. Vascular beta-adrenoceptor function in hypertension and in ageing. Can J Physiol Pharmacol. 2000;78:433452.

143. Zeiders JL, Seidler FJ, laccarino G, Koch WJ, Slotkin TA. Ontogeny of cardiac beta-adrenoceptor desensitization mechanisms: agonist treatment enhances receptor/G-protein transduction rather than eliciting uncoupling. J Mol Cell Cardiol. 1999;31:413-423.

144. Zeiders JL, Seidler FJ, Slotkin TA. Agonist-induced sensitization of beta-adrenoceptor signaling in neonatal rat heart: expression and catalytic activity of adenylyl cyclase. J Pharmacol Exp Ther. 1999;291:503-510.

145. Simonetta G, Rourke AK, Owens JA, Robinson JS, McMillen IC. Impact of placental restriction on the development of the sympathoadrenal system. Pediatr Res. 1997;42:805-811.

146. Suzuki $Y$, Huang $M$, Lederis $K$, Rorstad $O P$. The role of adenylate cyclase in relaxation of brain arteries: studies with forskolin. Brain Res. 1988;457:241-245. 
147. Ruijtenbeek $K_{\text {in }}$ Kessels $C G$, Janssen BJ, Bitsch $N J$, Fazzi GE, Janssen GM, De Mey J, Blanco CE. Chronic moderate hypoxia during in ovo development alters arterial reactivity in chickens. Pflugers Arch. 2003;447:158-167.

148. Bauer $R$, Walter $B$, Bauer $K$, Klupsch $R$, Patt $S$, Zwiener $U$. Intrauterine growth restriction reduces nephron number and renal excretory function in newborn piglets. Acta Physiol Scand. 2002;176:83-90.

149. Paixao AD, Maciel CR, Teles MB, Figueiredo-Silva J. Regional Brazilian diet-induced low birth weight is correlated with changes in renal hemodynamics and glomerular morphometry in adult age. Biol Neonate. 2001;80:239-246.

150. Manalich R, Reyes L, Herrera M, Melendi $C$, Fundora I. Relationship between weight at birth and the number and size of renal glomeruli in humans: a histomorphometric study. Kidney Int. 2000,58:770-773.

151. Koseki $C$, Herzlinger $D$, al-Awqati $Q$. Apoptosis in metanephric development. J Cell Biol. 1992;119:1327-1333.

152. Coles HS, Burne JF, Raff MC. Large-scale normal cell death in the developing rat kidney and its reduction by epidermal growth factor. Development. 1993;118:777-784.

153. Malik RK, Thornhill BA, Chang AY, Kiley SC, Chevalier RL. Apoptosis parallels ceramide content in the developing rat kidney. Pediatr Nephrol. 2000;15:188-191.

154. McPherson GA, Summers RJ. A study of alpha 1-adrenoceptors in rat renal cortex: comparison of $[3 \mathrm{H}]$-prazosin binding with the alpha 1-adrenoceptor modulating gluconeogenesis under physiological conditions. Br J Pharmacol. 1982;77:177-184.

155. Sanders M, Fazzi G, Janssen G, Blanco C, De Mey J. Prenatal stress changes rat arterial adrenergic reactivity in a regionally selective manner. Eur J Pharmacol. 2004:488:147-155.

156. Serio M, Montagnani M, Potenza MA, Mansi G, De Salvatore G, Mitolo-Chieppa D. Postnatal developmental changes of receptor responsiveness in rat mesenteric vascular bed. J Auton Pharmacol. 1996:16:63-68.

157. Battista MC, Oligny LL, St-Louis J, Brochu M. Intrauterine growth restriction in rats is associated with hypertension and renal dysfunction in adulthood. Am $J$ Physiol Endocrinol Metab. 2002;283:E124-131.

158. Fujinaga M, Scott JC. Gene expression of catecholamine synthesizing enzymes and beta adrenoceptor subtypes during rat embryogenesis. Neurosci Lett. 1997;231:108-112.

159. Fischer PA, Bogoliuk CB, Ramirez AJ, Sanchez RA, Masnatta LD. A new procedure for evaluation of renal function without urine collection in rat. Kidney int. 2000;58:1336-1341.

160. van Onna $M$, Houben $A J$, Kroon AA, Wierema TK, Koster D, van Engelshoven JM, de Leeuw PW. Asymmetry of renal blood flow in 
patients with moderate to severe hypertension. Hypertension. $2003 ; 41: 108-113$

161. Hill CE, Hirst GD, van Helden DF. Development of sympathetic innervation to proximal and distal arteries of the rat mesentery. $J$ Physiol. 1983;338:129-147.

162. Sanders MW, Fazzi GE, Janssen GM, de Leeuw PW, Blanco CE, De Mey JG. Reduced uteroplacental blood flow alters renal arterial reactivity and glomerular properties in the rat offspring. Hypertension. 2004;43:1283-1289.

163. Boero R, Pignataro A, Quarello F. Salt intake and kidney disease. $J$ Nephrol. 2002;15:225-229.

164. Bruun NE, Skott P. Damkjaer Nielsen M, Rasmussen S, Schutten $H J$, Leth $A$, Pedersen EB, Giese J. Normal renal tubular response to changes of sodium intake in hypertensive man. $J$ Hypertens. 1990;8:219-227.

165. Schmieder RE, Messerli FH, Garavaglia GE, Nunez BD. Dietary salt intake. A determinant of cardiac involvement in essential hypertension. Circulation. 1988;78:951-956.

166. du Cailar G, Ribstein J, Mimran A. Dietary sodium and target organ damage in essential hypertension. Am J Hypertens. 2002;15:222229.

167. Briscoe TA, Rehn AE, Dieni S, Duncan JR, Wlodek ME, Owens JA, Rees SM. Cardiovascular and renal disease in the adolescent guinea pig after chronic placental insufficiency. Am $J$ Obstet Gynecol. 2004;191:847-855.

168. Hales $\mathrm{CN}$, Ozanne SE. The dangerous road of catch-up growth. $J$ Physiol. 2003;547:5-10.

169. Mogensen CE. Glomerular filtration rate and renal plasma flow in short-term and long-term juvenile diabetes mellitus. Scand $\mathrm{J}$ Clin Lab invest. 1971:28:91-100.

170. Harris RC, Zhang MZ, Cheng HF. Cyclooxygenase-2 and the renal renin-angiotensin system. Acta Physiol Scand. 2004;181:543-547.

171. Miller JA. Renal responses to sodium restriction in patients with early diabetes mellitus. J Am Soc Nephrol. 1997;8:749-755.

172. Vallon $V$, Wead $L M$, Blantz RC. Renal hemodynamics and plasma and kidney angiotensin $\|$ in established diabetes mellitus in rats: effect of sodium and salt restriction. $J A m$ Soc Nephrol. 1995:5:1761-1767.

173. Thomson SC, Vallon V, Blantz RC. Kidney function in early diabetes: the tubular hypothesis of glomerular filtration. Am J Physio/ Renal Physiol. 2004;286:F8-15.

174. Vallon V, Kirschenmann D, Wead LM, Lortie MJ, Satriano J, Blantz $\mathrm{RC}$, Thomson SC. Effect of chronic salt loading on kidney function in early and established diabetes mellitus in rats. $J$ Lab Clin Med. 1997;130:76-82. 
175. Vallon V. Blantz RC, Thomson S. Glomerular hyperfiltration and the salt paradox in early [corrected] type 1 diabetes mellitus: a tubulocentric view. I Am Soc Nephrol. 2003;14:530-537.

176. van Paassen $P$, de Zeeuw $D$, Navis $G$, de Jong PE. Does the reninangiotensin system determine the renal and systemic hemodynamic response to sodium in patients with essential hypertension? Hypertension. 1996;27:202-208.

177. Williams $\mathrm{GH}$, Hollenberg NK. Are non-modulating patients with essential hypertension a distinct subgroup? Implications for therapy. Am J Med. 1985;79:3-9.

178. Strazzullo P, Barbato A, Vuotto P, Galletti F. Relationships between salt sensitivity of blood pressure and sympathetic nervous system activity: a short review of evidence. Clin Exp Hypertens. 2001;23:2533.

179. Mathieson PW. The cellular basis of albuminuria. Clin Sci (Lond). 2004;107:533-538.

180. Russo LM, Bakris GL, Comper WD. Renal handling of albumin: a critical review of basic concepts and perspective. Am J Kidney Dis. 2002;39:899-919.

181. Russo LM, Comper WD, Osicka TM. Mechanism of albuminuria associated with cardiovascular disease and kidney disease. Kidney Int Suppl. 2004:S67-68.

182. Russo LM, Osicka TM, Bonnet F, Jerums G, Comper WD. Albuminuria in hypertension is linked to altered lysosomal activity and TGF-beta1 expression. Hypertension. 2002;39:281-286.

183. Yu HC, Burrell LM, Black MJ, Wu LL, Dilley RJ, Cooper ME, Johnston $\mathrm{Cl}$. Salt induces myocardial and renal fibrosis in normotensive and hypertensive rats. Circulation. 1998;98:26212628.

184. Yuan BX, Leenen FH. Dietary sodium intake and left ventricular hypertrophy in normotensive rats. Am J Physiol. 1991;261:H13971401.

185. Musiari L, Ceriati $R$, Taliani U, Montesi M, Novarini A. Early abnormalities in left ventricular diastolic function of sodium-sensitive hypertensive patients. J Hum Hypertens. 1999;13:711-716.

186. Braaksma MA, Dassel AC, Aarnoudse JG. Renal responses to prolonged $(48 \mathrm{~h})$ hypoxemia without acidemia in the late-gestation ovine fetus. Am J Physiol. 1999;277:R395-402.

187. Solhaug $\mathrm{MJ}$, Bolger $\mathrm{PM}_{\text {, Jose } P A}$. The developing kidney and environmental toxins. Pediatrics. 2004;113:1084-1091.

188. Chen $Y$, Lasaitiene $D$, Friberg $P$. The renin-angiatensin system in kidney development. Acta Physiol Scand. 2004;181:529-535.

189. Woods LL, Rasch R. Perinatal ANG II programs adult blood pressure, glomerular number, and renal function in rats. Am J Physiol. 1998;275:R1593-1599. 
190. Dell'Omo G, Penno $G_{*}$ Giorgi D, Di Bello V, Mariani M, Pedrinelli R. Association between high-normal albuminuria and risk factors for cardiovascular and renal disease in essential hypertensive men. Am $J$ Kidney Dis. 2002;40:1-8.

191. Hostetter TH, Olson $J$, Rennke HG, Venkatachalam MA, Brenner BM. Hyperfiltration in remnant nephrons: a potentially adverse response to renal ablation. $J$ Am Soc Nephrol. 2001;12:1315-1325.

192. Woods LL, Weeks DA, Rasch R. Hypertension after neonatal uninephrectomy in rats precedes glomerular damage. Hypertension. 2001;38:337-342.

193. Woods LL. Neonatal uninephrectomy causes hypertension in adult rats. Am J Physiol. 1999;276:R974-978.

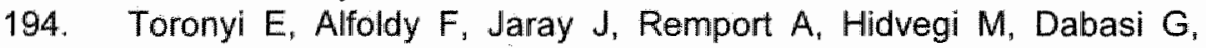
Telkes G, Offenbacher E, Perner F. Evaluation of the state of health of living related kidney transplantation donors. Transp/ Int. 1998;11 Suppl 1:S57-59.

195. Praga M, Hernandez E, Herrero JC, Morales E, Revilla Y, DiazGonzalez R, Rodicio JL. Influence of obesity on the appearance of proteinuria and renal insufficiency after unilateral nephrectomy. Kidney Int. 2000;58:2111-2118.

196. Manning RD, Jr., Hu L, Tan DY, Meng S. Role of abnormal nitric oxide systems in salt-sensitive hypertension. Am \& Hypertens. 2001;14:68S-73S.

197. Cotran RS, Rennke HG. Anionic sites and the mechanisms of proteinuria. N Engl J Med. 1983;309:1050-1052.

198. Vallon V, Huang DY, Deng A, Richter K, Blantz RC, Thomson S. Salt-sensitivity of proximal reabsorption alters macula densa salt and explains the paradoxical effect of dietary salt on glomerular filtration rate in diabetes meliitus. J Am Soc Nephrol. 2002;13:1865-1871. 


\section{Summary}

It has been proposed that an insufficient delivery of nutrients and oxygen to the fetus can limit intrauterine growth and increase the risk for hypertension and other (cardiovascular) diseases in adult life.

This thesis describes studies designed to investigate the prolonged effect of maternal uterine blood flow restriction on the offspring, with particular emphasis on effects on the development of vascular adrenergic reactivity and renal structure and function.

We first investigated normal development of adrenergic, sympathetic and peptidergic reactivity in arteries from different regions of the vascular bed. The data presented in chapter 2 demonstrate that several parts of the vascular tree are innervated to a different extend during development and the maturation of adrenergic and peptidergic responses occurs in a distinct way in different parts of the vascular tree. Ingrowing sympathetic and sensory motor nerves may redistribute receptors on the cell surface or can be involved in the post receptor expression of G-proteins or the subsequent signal transduction system. These findings were important to understand the findings of the studies outlined in chapter 3 and 4.

In chapter 3 and 4 we describe the effects of experimental intrauterine stress (IUS), induced by a bilateral ligation of the uterine arteries, at an early (day 13 of pregnancy) and later (day 17 of pregnancy) stage of gestation. An early intervention during pregnancy only altered renal arterial adrenergic reactivity, while other areas of the vascular tree were not affected (chapter 3). An intervention at a later stage of gestation emphasizes these regional differences; they were not only confined to renal arteries, but were even more evident in right than in the left renal arteries (chapter 4). However, we found that IUS induced at different time points of fetal development and the possible subsequent catch-up growth have dissimilar effects on the maturation of vascular adrenergic reactivity and of the structure of the kidneys. Although both time points of intervention altered renal arterial responses to $\beta$-adrenergic activation, modification of $\alpha_{1}$ adrenergic mediated responses were only modified as a result of the early intervention at day 13 of gestation. Additionally, we observed that $\beta$ adrenergic activity is regulated by different mechanisms, depending on the time point of intervention. In contrast to the intervention at day 13 , the signal transduction system is involved in the enhanced response to $\beta$-adrenoceptor stimulation after the late intervention at day 17.

Furthermore, the late induction of IUS led to growth retardation, while the early induction of IUS led to prenatal growth acceleration (catch-up growth). 
Since birth weight seems only crude evidence for a suboptimal fetal environment, we also measured hematocrit values in newborn rats that survived IUS induced at day 17 of pregnancy. Hematocrit values of these rats were increased after IUS, indicating that the fetal environment was more hypoxic than normal. The discrepancies between the two time points of intervention were further highlighted by differences in their effects on renal structure. IUS induced at day 17 of gestation led to a decrease in glomerular density and glomerular hypertrophy, which persisted throughout adulthood. We could not observe these changes in the kidneys of rats that survived IUS, induced at day 13 .

The observed reduced nephron endowment eventually resulted in hyperfiltration, increased renal blood flow and increased filtration fraction in the kidneys of the adult rat. Moreover, we observed that these rats developed albuminuria, which is further evidence for a disturbed renal function as a result of IUS (chapter 4 ).

We also investigated how the IUS induced changes in glomerular structure and hemodynamic function can be affected by challenges like an additional reduction of the number of glomeruli by unilateral nephrectomy or by a high sodium intake (chapter 4 and 5). After the nephrectomy, we observed an increase in blood pressure in animals that survived IUS. It also resulted in severe renal insufficiency, as indicated by the high plasma creatinine concentrations and a reduced renal compensatory capacity in the adult rat.

A high sodium diet also resulted in an increased blood pressure and again we found that animals that survived IUS had lost their ability to adapt their renal function to this challenge. High sodium further increased the urinary albumin concentrations and worsened the albuminuria in animals that survived IUS.

This thesis documents that the development of renal arterial reactivity and renal structure and function can be perturbed in an animal model of fetal programming. The data presented also provide evidence for an IUS induced increased susceptibility to develop hypertension, due to life style factors, such as high sodium intake. 


\section{Samenvatting}

Hart- en vaatziekten zijn een groot probleem voor de volksgezondheid, waarbij roken, overgewicht, weinig lichaamsbeweging, diabetes mellitus en een genetische achtergrond de belangrijkste risicofactoren zijn. Uit onderzoeken in mens en dier is gebleken dat, behalve genetische factoren, ook omgevingsfactoren vóór de geboorte van belang kunnen zijn voor het ontstaan van hypertensie, hart- en vaatziekten en diabetes mellitus. Een onvoldoende aanvoer van voedingsstoffen en zuurstof naar de foetus kunnen de groei van de foetus verstoren en de ontwikkeling van weefsels en organen, zoals bloedvaten en nieren permanent veranderen. Deze veranderingen kunnen de kans verhogen op het ontwikkelen van hart- en vaatziekten op latere leeftijd. Dit gebeurt in een proces dat men foetale programmering noemt. De mechanismen die verantwoordelijk zijn voor de programmering van hart- en vaatziekten zijn nog niet opgehelderd, maar om meer inzicht te krijgen in deze mechanismen wordt gebruik gemaakt van diermodellen, zoals de rat.

In onze westerse maatschappij wordt, in de mens, een onvoldoende aanvoer van voedingsstoffen en zuurstof vooral veroorzaakt door een slecht werkende placenta (placentaire insufficiëntie). In de studies beschreven in dit proefschrift werd deze placentaire insufficiëntie nagebootst in de rat. Dit werd bereikt door tijdens de zwangerschap, de bloedvaten, die de baarmoeder voorzien van bloed, af te binden en zo de toevoer van bloed naar de foetus te beperken. Dit leidt tot een verstoorde groei van de nakomelingen en een verhoogde hematocrietwaarde (\% rode bloedcellen) in het bloed. Het laatste suggereert dat de nakomelingen zich ontwikkelden in een omgeving met een lage zuurstofspanning.

In hoofdstuk 3 laten we zien dat, na placentaire insufficièntie, de reactiviteit van de bloedvaten naar de nier van jonge ratten is veranderd ten opzichte van de normale situatie (hoofdstuk 2). Placentaire insufficiëntie leidt echter niet tot een verstoorde reactiviteit van bloedvaten elders in de circulatie. Als reactie op ongunstige omstandigheden in de baarmoeder, probeert de foetus ervoor te zorgen dat vitale organen voldoende zuurstof en voedingsstoffen krijgen. Er vindt redistributie van de bloedstroom plaats, ten gunste van bijvoorbeeld de hersenen, maar ten koste van andere organen. De nieren worden in een dergelijk situatie minder doorbloed en krijgen daardoor minder zuurstof en voedingsstoffen. Als gevolg daarvan kunnen er tijdens de ontwikkeling van de nieren structurele en functionele veranderingen ontstaan, zoals in de bloedvaten, waardoor er minder glomeruli (filtereenheden van nieren) worden aangelegd. 
Uit de resultaten van studies in jong volwassen ratten blijkt dat er inderdaad minder glomeruli zijn aangelegd in de nieren van nakomelingen van een zwangerschap met placentataire insufficiëntie (hoofdstuk 4). Deze structurele veranderingen hebben vergaande consequenties voor de functie van de nieren. Om met een lager aantal glomeruli een normale nierfunctie te: waarborgen, verhoogt de bloedtoevoer naar de nieren en gaan de glomeruli meer filtreren (glomerulaire hyperfiltratie). De dieren hebben geen verhoogde bloeddruk, maar er wordt wel een verhoogde concentratie albumine in de urine aangetroffen. Dit wijst op een verminderde nierfunctie en wordt in verband gebracht met een verhoogde kans op het krijgen van hart-en vaatziekten.

Wanneer één van de nieren wordt verwijderd, blijkt dat de overgebleven nier van ratten die placentaire insufficiëntie hebben ondergaan, niet kan compenseren voor het verlies van de andere nier, terwijl de overgebleven nier van normale controle ratten dit wel kan (hoofdstuk 4). Daarnaast hebben deze ratten een verhoogde bloeddruk, een nog verder verhoogde concentratie albumine in de urine en dus een nog verdere achteruitgang van de nierfunctie.

Om na te gaan of nakomelingen van een zwangerschap met placentaire insufficiëntie een verhoogd risico hebben op het krijgen van harten vaatziekten als gevolg van leefstijlfactoren, zoals hoge zoutinname, kregen de volwassen ratten een dieet met een hoge concentratie zout in het drinkwater (hoofdstuk 5). Uit deze studie blijkt dat een dieet met een hoge concentratie zout, na placentaire insufficiëntie, resulteert in een verstoorde nierfunctie, die vergelijkbaar is met het verwijderen van een nier. Tevens heeft een hoge zoutinname tot gevolg dat de bloeddruk stijgt en de concentratie albumine in de urine verder toeneemt.

Dit proefschrift beschrijft dat een beperkte toevoer van bloed naar de foetus leidt tot een verstoorde foetale groei en vergaande gevolgen heeft voor de reactiviteit van de bloedvaten, de structuur en de functie van de nier. Het is dus van groot belang factoren die de groei van de foetus en de ontwikkeling van de nier belemmeren, te mijden tijdens de zwangerschap. In de toekomst is het belangrijk dat kinderartsen kinderen, die een verhoogde kans hebben op het krijgen van hart- en vaatziekten op tijd herkennen om hen goed te controleren en adequaat te behandelen. Men kan hierbij denken aan medicatie die erop gericht is de functie van de nieren te optimaliseren na een veranderde structuur en functie van de nier. Daarnaast kunnen ongunstige invloeden tijdens de zwangerschap personen gevoeliger maken voor leefstijlfactoren, zoals hoge zoutinname. 


\section{Dankwoord}

"Promoveren? .........Nee, dat is niets voor mij": dacht ik. Maar na een jaartje Jo \& Co was ik van mening veranderd. Jo, jij hebt me overgehaald aan dit project te beginnen en mij de kans geboden leuke en mooie stukken van de (wetenschappelijke) wereld te zien. Daar ben ik je heel dankbaar voor. Your door is always open and so is your mind. Ik heb ontzettend veel van je geleerd en dat doe ik nog steeds. Jouw hart voor het onderzoek en je " $\mathrm{Co}$ " is bewonderenswaardig.

Carlos, jou wil ik bedanken voor je klinische input, en je grote interesse in mijn onderzoek. Daarnaast heb je de capaciteit om de juiste vragen te stellen en ook daar heb ik veel van geleerd.

Jo \& Co zou niet zijn wat het is zonder "de harde kern", Paul, Ger en Gregorio.

Paul, een betere kamergenoot had ik me niet kunnen wensen. Lief en leed kan ik met je delen of het nu gaat over onderzoek, wateroverlast of lekker eten en drinken. Je hebt een engelengeduld en je bent nooit te beroerd om te helpen of om een vraag te beantwoorden. Via jou heb ik ook de hulp van Jet gekregen.

Jet, de PAH/inuline bepalingen die jij voor mij hebt gedaan waren onmisbaar voor mijn onderzoek. Bedankt voor je hulp!

Ger, de stille kracht van het lab. "Ich höb dich vaak de pis lauw gemaak" met albumine bepalingen, maar ik heb je nooit horen klagen. Vooral waardeer is je droge gevoel voor humor en je verassende opmerkingen. Je bent uniek!

Fazzi (Gregorio voor intimi), niet voor niets heb je gevraagd één van mijn paranimfen te zijn. Behalve het ontelbare aantal glomeruli, dat je toch wist te tellen en te meten, heb je menig computerprobleem voor me weten op te lossen. Maar bovenal houd ik van "typische" mensen en daar ben jij er zeker één van. Je typische gevoel voor humor, je typische dance moves (ook op het lab) en je typische en diepgaande interesse voor je familiehistorie zijn slechts voorbeelden. Gregorio, bedankt voor alles en ik hoop dat je goed kunt aarden op mijn geboortegrond!

Goed voorbeeld doet goed volgen. Mariëlle, Liesje, Karin en Wendy zijn mij voorgegaan en hebben laten zien hoe het moet. Karin, zoals je zelf al schreef, samen met fetal programming bezig en samen op congres. Dankzij jou waren de congressen onvergetelijk en heb ik veel gelachen. Ik wens je veel geluk met je aanstaande moederschap en denk erom goed doorademen en gezond eten! Wendy, ik bewonder je enorme doorzettingsvermogen en mis onze goede gesprekken. Alle geluk voor de toekomst! 
Zij die mij volgen zijn Tijl, Veerle, Suzanne en Geraldine. Veel succes en maak er wat van!

Geraldine, most of all, you have become a good friend! We had and still have a lot of fun. Thanks for all the help, especially during the last phase of my promotion. It is an honour to have you as one of my paranimfen. Good luck with your own promotion and with your plans for the future. Merci beaucoup!

Ook andere mensen van de afdeling wil ik bedanken voor een fijne tijd. en veel gezelligheid.

Nicole, allereerst hielp je me altijd met mijn soms agressieve ratten. Jij wist ze altijd wel te temmen. Daarnaast kwam je altijd even langs op het lab met een vrolijk "Ha meisje, hoe gaat het?". Zo ben je nu eenmaal en dat maakt je onmisbaar!

Rob en Helma, bedankt voor de gezelligheid, jullie goede raad en adviezen.

Mia, Els en Mariëlle, bedankt voor jullie hartelijkheid, hulp en luisterende oren.

Ten slotte mogen mijn ouders en Marcel natuurlijk niet ontbreken in mijn woord van dank.

Pap en Mam, geer höb os een fijn jeug en veul wies lesse mitgegeve. Veural: "Es se urges aan begins, maaks se 't auch aaf". Det is noe gelök. Bedank veur allies!

Marcel, teagewèndj en 'n bietje aafzeen huërt d'rbie, mer doe heels mich op koers. Op dich (en mit dich) ken ich boewe! 


\section{Curriculum vitae}

Name

Date of birth

Place of birth

\author{
Maria Wilhelmina Buckx-Sanders \\ January 11, 1972 \\ Susteren, The Netherlands
}

Education

1984-1991 Gymnasium $\beta$, College Sittard, The Netherlands

1991-1992 Biology, Katholieke Universiteit Nijmegen, the Netherlands

1992-1997 (Biological) Health Sciences, University of Maastricht, The Netherlands

Research

1998-1999 Research Assistant, Dept. of Pharmacology \& Toxicology, University of Maastricht, The Netherlands

1999-2005 PhD student, Dept. of Pharmacology \& Toxicology, Cardiovascular Research Institute Maastricht (CARIM), University of Maastricht, The Netherlands

2005 Postdoc, Dept. of Pharmacology \& Toxicology, Cardiovascular Research Institute Maastricht (CARIM), University of Maastricht, The Netherlands

Awards

Burroughs Wellcome Fund Poster Award, FASEB Summer Research Conference on Perinatal Regulation of the Cardiovascular System, 4-9 juni 2000. Coppermountain, CO, USA.

Young Scientist Travel Award, Vascular Neuroeffector Mechanisms, 12-15 juli 2002, Granlibakken, Lake Tahoe, CA, USA.

Scholarships, Annual meeting of the European Council for Blood Pressure and Cardiovascular Research (ECCR),

oktober 2000 , Noordwijkerhout, the Nethererlands,

oktober 2001, Noordwijkerhout, the Netherlands

oktober 2003, Seeheim, Germany

oktober 2004, Nice, France 


\section{Publications}

Sanders MW, Nieuwenhulys CM, Feijge MA, Rook M, Beguin S, Heemskerk JW. The procoagulant effect of thrombin on fibrin(ogen)-bound platelets. Haemostasis. $1998 ; 28: 289-300$.

Sanders MW, Fazzi GE, Janssen GMJ, Blanco CE, De Mey JGR. Prenatal stress changes rat arterial adrenergic reactivity in a regionally selective manner. European Journal of Pharmacology. 2004; 488: 147.

Sanders MW, Fazzi GE, Janssen GMJ, de Leeuw PW, Blanco CE, De Mey JGR. Reduced uteroplacental blood flow alters renal arterial reactivity and glomerular properties in the rat offspring. Hypertension. 2004; 43(6): 12831289.

Sanders MW, Fazzi GE, Janssen GMJ, Blanco CE, De Mey JGR. High sodium intake increases blood pressure and alters renal function in intrauterine growth retarded rats. Hypertension. 2004; 46(1): 71-75. 